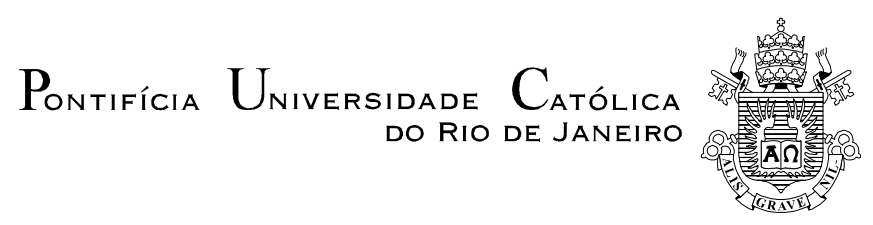

William Schroeder Cardozo

\title{
Numerical and experimental study of a two degrees of freedom electrohydraulic manipulator
}

TESE DE DOUTORADO

Thesis presented to the Programa de Pós-graduação em Engenharia Mecânica of PUC-Rio in partial fulfillment of the requirements for the degree of Doutor em Ciências Engenharia Mecânica.

Advisor: Prof. Hans Ingo Weber

Rio de Janeiro July 2017 


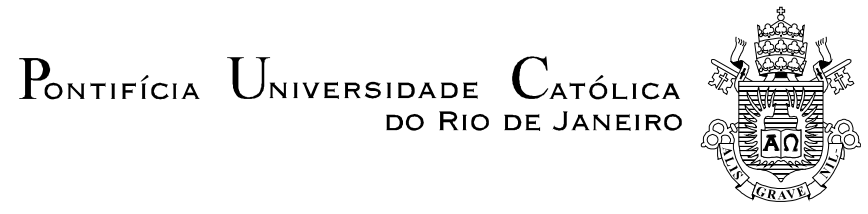

William Schroeder Cardozo

\section{Numerical and experimental study of a two degrees of freedom electrohydraulic manipulator}

Thesis presented to the Programa de Pós-Graduação em Engenharia Mecânica of the Departamento de Engenharia Mecânica, PUC-Rio as a partial fulfillment of the requirements for the degree of Doutor.

Prof. Hans Ingo Weber Advisor Departamento de Engenharia Mecânica - PUC-Rio

Prof. Marco Antonio Meggiolaro

Departamento de Engenharia Mecânica - PUC-Rio

Prof. Roberta de Queiroz Lima

Departamento de Engenharia Mecânica - PUC-Rio

Prof. Max Suell Dutra UFRJ

Prof. Agenor de Toledo Fleury USP

Prof. Márcio da Silveira Carvalho Vice Dean of Graduate Studies Técnico Científico - PUC-Rio

Rio de Janeiro, July 26th, 2017 
All rights reserved.

\section{William Schroeder Cardozo}

William Cardozo graduated as mechanical engineer in 2006 from PUC-Rio (Rio de Janeiro, RJ), and he got his master in 2012 from the same institution. Worked for seven years developing grinding machine tools and two years developing a vehicle telemetry system.

Ficha Catalográfica

Cardozo, William Schroeder

Numerical and experimental study of a two degrees of freedom electrohydraulic manipulator / William Schroeder Cardozo; advisor: Hans Ingo Weber. - 2017. 116 f. : il. (color.) ; $30 \mathrm{~cm}$

Tese (doutorado)-Pontifícia Universidade Católica do Rio de Janeiro, Departamento de Engenharia Mecânica, 2017.

Inclui bibliografia

1. Engenharia mecânica - Teses. 2. Sistema Embarcado. 3. Manipuladores robóticos. 4. Atuador eletro-hidráulico. 5. Controle de atitude. I. Weber, Hans Ingo. II. Pontifícia Universidade Católica do Rio de Janeiro. Departamento de Engenharia Mecânica. III. Título.

CDD: 621 
To Aline and Henry 


\section{Acknowledgements}

Firstly, I would like to thank my advisor, Prof. Hans Ingo Weber, for your support over the past four years.

I would like to express my gratitude to the members of my examination committee.

To Romulo Reis Aguiar for your attendance on the day of the defense and for your review of this Thesis.

To my parents for the education, love and support.

To Aline and Henry for the love and patience.

To Timo Wekerle for your help with the references and for your review.

To all the professors and staff of the Department for the teachings and the help.

To all the friends and family that in one way or another stimulated or helped me.

I thank PUC-Rio and CNPq for the aid granted, without which this work could not have been carried out. 


\section{Abstract}

Cardozo, William Schroeder; Weber, Hans Ingo (Advisor). Numerical and experimental study of a two degrees of freedom electrohydraulic manipulator. Rio de Janeiro, 2017. 116p. D.sc. Thesis - Departamento de Engenharia Mecânica, Pontifícia Universidade Católica do Rio de Janeiro.

Thrust Vector Control (TVC) is used for the attitude control of spacecrafts. In the case of liquid-propellant fuel, the nozzle is traditionally connected to the rocket frame through a gimbal. Two hydraulic actuators are placed around the nozzle to control its orientation. In this Thesis, TVC is treated as a fixed base robotic platform. Instead of using commercial servo-valves to control the actuators, a novel control valve is proposed. First a gimbaled platform is considered with two angular position transducers to measure the angular displacement of the joint crosshead. Then, a homokinetic platform configuration is proposed replacing the gimbal by a constant velocity joint. In this case, the platform position feedback is done using a real-time attitude estimator. The estimator is a complementary filter based on orientation matrices that collects data from an inertial measurement unit (IMU). The modeling of the system begins with kinematics. Then, the dynamic modeling uses the Newton-Euler formulation to obtain the equation of motion. The modeling of the electro-hydraulic system is presented with the model of the novel control valve and the linear actuator. Initially, a full proportional controller is proposed. During the experimental validation it is shown that due to the characteristics of the actuation system, even this simple controller is accurate and reliable. Thereafter, method is demonstrated to evaluate novel control strategies. The comparison between the gimbaled and homokinetic platform shows that, under the analyzed conditions, they have a similar dynamic behavior. In both platform configurations the system is accurate and reliable.

\section{Keywords}

Dynamics; Robotic Manipulators; Electro-hydraulic actuators; Position control. 


\section{Resumo}

Cardozo, William Schroeder; Weber, Hans Ingo. Estudo numérico e experimental de um manipulador eletro-hidráulico de dois graus de liberdade. Rio de Janeiro, 2017. 116p. Tese de doutorado - Departamento de Engenharia Mecânica, Pontifícia Universidade Católica do Rio de Janeiro.

O controle de empuxo vetorial (TVC) é usado para o controle de atitude de foguetes aeroespaciais. No caso de propulsão usando combustível líquido, tradicionalmente o bocal é conectado ao corpo do foguete através de uma junta cardânica. Dois atuadores hidráulicos são colocados ao redor do bocal para controlar sua orientação. Nesta tese, o TVC é tratado como uma plataforma robótica de base fixa. Ao invés de usar servo-válvulas comerciais para controlar os atuadores, uma nova válvula de controle é proposta. Primeiro uma plataforma cardânica é considerada com transdutores de posição angular medindo o deslocamento da cruzeta da junta. Em seguida, uma nova configuração da plataforma é proposta substituindo o cardan por uma junta homocinética. Neste caso, a realimentação da posição da plataforma é feito usando um estimador de atitude em tempo real. Este estimador é um filtro complementar baseado em matrizes de orientação que coleta dados de uma central inercial (IMU). A modelagem do sistema começa com a cinemática. Na sequiência, a modelagem dinâmica utiliza a formulação de Newton-Euler para obter a equação de movimento. A modelagem do sistema hidráulico é apresentada com o modelo da nova válvula de controle e do atuador. Inicialmente, um controlador puramente proporcional é proposto. Durante a validação experimental é mostrado que devido as características do sistema de atuação, mesmo este simples controlador é preciso e confiável. Em seguida é demonstrado um método para avaliar outras estratégias de controle. A comparação entre a plataforma cardânica e homocinética mostra que, nas condições analisadas, ambas têm um comportamento dinâmico similar. Nas duas configurações da plataforma o sistema se mostrou preciso e confiável.

\section{Palavras-chave}

Dinâmica de rotação; Manipuladores Robóticos; Atuadores hidráulicos; Controle de Posição. 


\section{Contents}

1 Introduction 18

1.1. Fundamentals of TVC 18

1.2. Literature review 20

1.3. Objective of this Thesis and summary of results 23

1.4. Outline of this Thesis 24

2 Fundamentals of the kinematic and dynamic analysis 26

2.1. The rigid body motion 26

2.2. About the nomenclature 27

2.3. Vector product and the tilde matrix 28

$\begin{array}{ll}\text { 2.4. The attitude matrix } & 28\end{array}$

2.5. Angular velocity definition 30

2.6. Attitude matrix obtained by sequential rotations 31

2.7. Attitude matrix as rotation matrix 32

3 Platform development $\quad 35$

3.1. Gimbaled platform 36

3.2. Homokinetic platform 41

4 Hydraulic control system $\quad 48$

4.1. Actuator model 50

4.2. Valve model 51

4.3. Other model considerations 56

5 Data acquisition and control system 59

5.1. DAQ system 59

5.2. Controller 60

5.3. Sensors 62

5.4. Attitude estimation 64

5.5. About the simulations 67 
6 Results 68

6.1. Gimbaled platform 68

6.2. Homokinetic platform 81

6.3. Comparison between gimbaled and homokinetic platform 83

6.4. Various load conditions on the gimbaled platform 90

6.5. Controller 94

7 Conclusion and suggestions for future work 98

$\begin{array}{ll}\text { 7.1. Future works } & 100\end{array}$

$\begin{array}{ll}\text { A Preliminary test benches } & 106\end{array}$

$\begin{array}{ll}\text { A.1 No load test bench } & 106\end{array}$

A.2 Inverted pendulum test bench 106

$\begin{array}{ll}\text { A.3 Actuator friction identification } & 107\end{array}$

$\begin{array}{ll}\text { B Electrohydraulic circuit } & 109\end{array}$

$\begin{array}{ll}\text { C Data acquisition system } & 111\end{array}$

D Rotation around a vector 114 


\section{List of Figures}

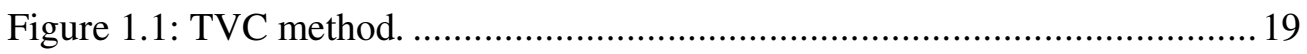

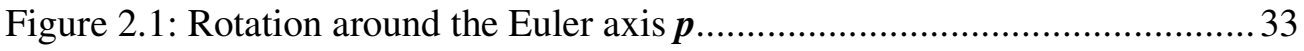

Figure 3.1: Platform overview with the peripheral system............................... 35

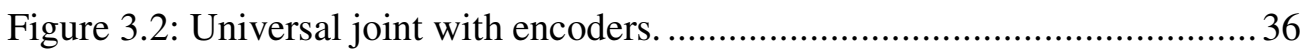

Figure 3.3: Gimbaled platform kinematic scheme. ......................................... 37

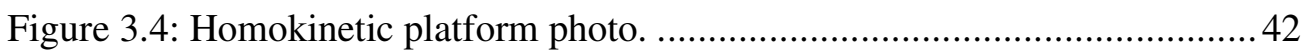

Figure 3.5: CVJ with aligned axes (left) and not aligned (right)...................... 43

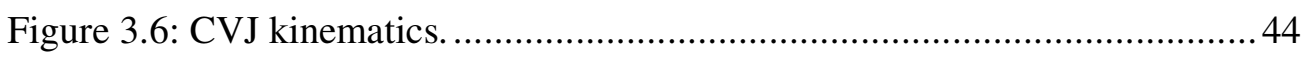

Figure 3.7: Homokinetic platform kinematic scheme..................................... 45

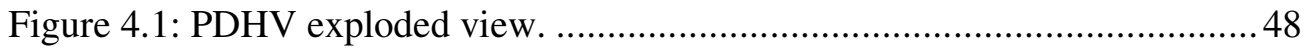

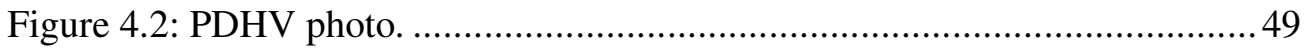

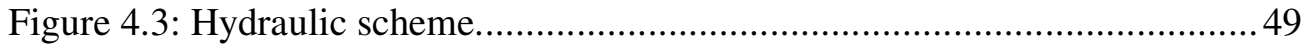

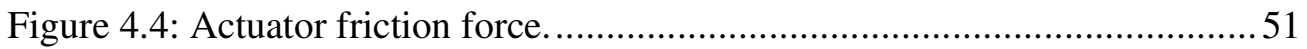

Figure 4.5: Orifice area as a function of the valve position.............................52

Figure 4.6: Valve opening in no-load experiment...........................................53

Figure 4.7: Chamber $A$ flow in no-load experiment. ........................................53

Figure 4.8: Chamber $B$ flow in no-load experiment. ........................................5 54

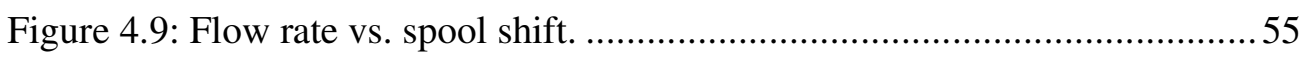

Figure 4.10: Valve shift limitation caused by the stepper controller...................56

Figure 4.11: Supply and chambers pressure during no-load experiment.............57

Figure 5.1: Electrical peripheral system......................................................59

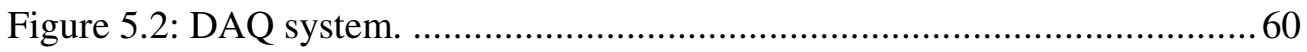

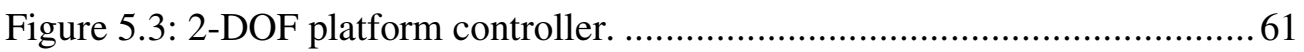

Figure 5.4: Step response of HA1 with different gains. ...................................62 62

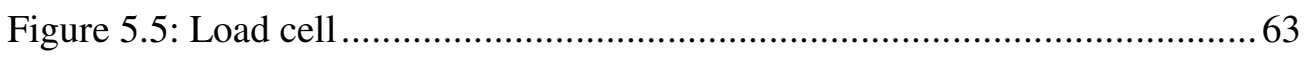

Figure 5.6: Attitude estimation validation with $\theta$ and $\phi$ angle.........................66

Figure 5.7: Attitude estimation validation with $\alpha$ and $\beta$ angle. ......................66

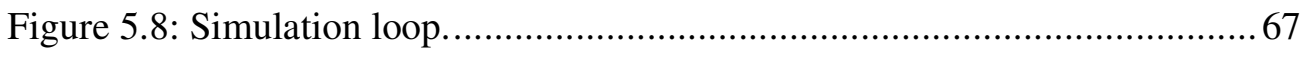

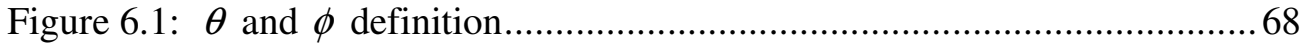

Figure 6.2: $\theta$ and $\phi$ result when alternating between two points..................... 71

Figure 6.3: $\alpha$ and $\beta$ results when alternating between two points.................. 71

Figure 6.4: Actuators length when alternating between two points. ................... 72 
Figure 6.5: Actuator force when alternating between two points....................... 73

Figure 6.6: Controller output when alternating between two points. .................. 73

Figure 6.7: Table center trajectory when alternating between two points............ 74

Figure 6.8: Table center trajectory for the tricuspoid. ..................................... 75

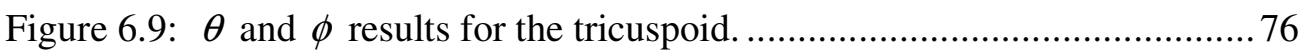

Figure 6.10: $\alpha$ and $\beta$ results for the tricuspoid........................................ 76

Figure 6.11: Actuator force for the tricuspoid................................................. 77

Figure 6.12: Controller output for the tricuspoid. ........................................... 77

Figure 6.13: Actuators length for the tricuspoid............................................. 78

Figure 6.14: Top view of the table center trace for the circular trajectory. .......... 79

Figure 6.15: Platform attitude for the circular trajectory ................................ 79

Figure 6.16: Actuators length for the circular trajectory. .................................. 80

Figure 6.17: Simulated and experimental homokinetic platform orientation. ...... 81

Figure 6.18: Homokinetic platform actuator length. ...................................... 82

Figure 6.19: Top view of the homokinetic platform table center trace. .............. 82

Figure 6.20: Attitude comparison between gimbaled and homokinetic platform. 83

Figure 6.21: Force comparison between gimbaled and homokinetic platform..... 84

Figure 6.22: HA1 chambers pressure comparison............................................ 85

Figure 6.23: HA2 chambers pressure comparison........................................... 85

Figure 6.24: Numerical results for the platforms spin rate. …........................ 87

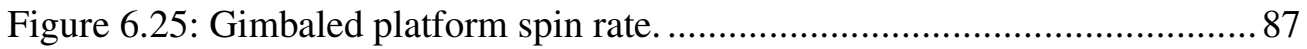

Figure 6.26: Homokinetic platform spin rate. ............................................. 88

Figure 6.27: Experimental results for the platforms spin rate........................... 89

Figure 6.28: Moment done on $\mathrm{z}$ direction by the fixed base............................... 90

Figure 6.29: Actuators forces with different loads. .......................................... 91

Figure 6.30: Controller output on different loads.......................................... 92

Figure 6.31: Actuators displacement on different loads.................................. 92

Figure 6.32: Force for different loads on a wider trajectory. ............................ 93

Figure 6.33: Controller output for different load on a wider trajectory ............... 93

Figure 6.34: Actuators displacement for different loads on a wider trajectory..... 94

Figure 6.35: Table center trajectory with the PI controller............................... 95

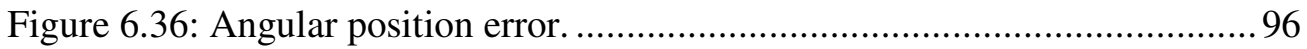

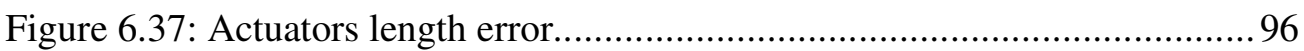




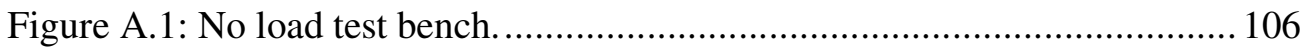

Figure A.2: Inverted pendulum test bench................................................ 107

Figure A.3: Four different friction estimations. ............................................. 108

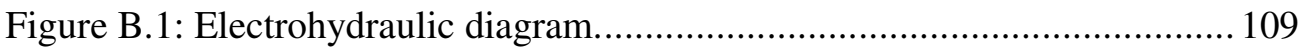

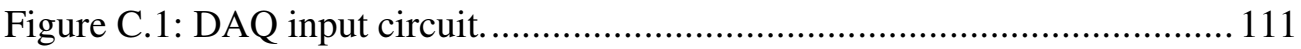

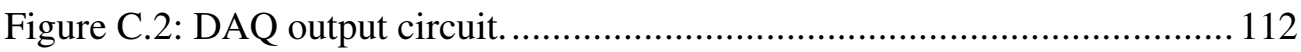

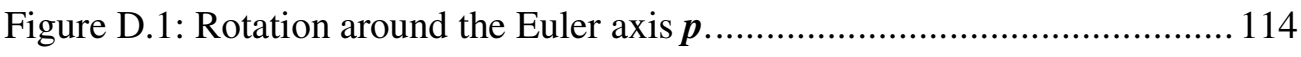

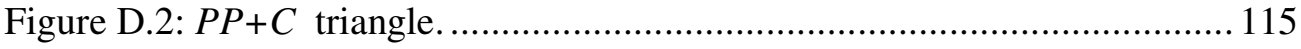

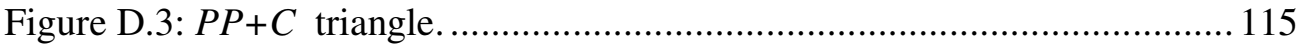




\section{Notation}

$A_{e} \quad$ Area of the embolus.

$A_{c} \quad$ Area of the crow.

$B \quad$ Center of the fixed base.

$C \quad$ Center of the top surface of the moving platform.

$C_{31} \quad$ Center of the HA1 ball joint.

$C_{32} \quad$ Center of the HA2 ball joint.

$C_{21} \quad$ Center of the crosshead of the HA1 universal joint.

$C_{22} \quad$ Center of the crosshead of the HA2 universal joint.

$\boldsymbol{E} \quad 3 \times 3$ identity matrix.

$F \quad$ Reference frame of the fixed base

$F_{f} \quad$ Actuator friction force.

${ }^{F} \boldsymbol{F}_{a 1} \quad$ Force on the HA1 using $F$ coordinates.

${ }^{F} \boldsymbol{F}_{a 2} \quad$ Force on the HA2 using $F$ coordinates.

$F_{a 1} \quad$ Force magnitude on the HA1.

$F_{a 2} \quad$ Force magnitude on the HA2.

${ }^{S} \boldsymbol{G}_{L} \quad$ Vector of linear momentum of the load using $S$ coordinates.

${ }^{S} \boldsymbol{G}_{P} \quad$ Vector of linear momentum of the moving platform using $S$ coordinates.

${ }^{S} \boldsymbol{H}_{L} \quad$ Angular momentum of the load about its CM using $S$ frame.

${ }^{S} \boldsymbol{H}_{O} \quad$ Angular momentum of the system about the point $O$ using $S$ frame.

${ }^{S} \boldsymbol{H}_{P} \quad$ Angular momentum of the moving platform about its CM using $S$ frame.

${ }^{S} \boldsymbol{I}_{P} \quad$ Inertia matrix of the moving platform about its CM using $S$ coordinates.

${ }^{S} \boldsymbol{I}_{L} \quad$ Inertia matrix of the load about its CM using $S$ coordinates.

$I_{1 P} \quad$ First and second principal moment of inertia of the moving platform about its

CM.

$I_{3 P} \quad$ Third principal moment of inertia of the moving platform about its CM.

$I_{1 L} \quad$ First and second principal moment of inertia of the moving load about its CM.

$I_{3 L} \quad$ Third principal moment of inertia of the moving load about its CM.

$L \quad$ CM of the load platform

${ }^{S} \boldsymbol{M}_{O} \quad$ Moment acting on the moving platform using $S$ frame.

${ }^{S} \underline{\boldsymbol{M}}_{J} \quad$ Torque vector of the joint using $S$ frame.

$O \quad$ Center of the crosshead on the gimbaled platform or center of the CVJ on the homokinetic platform.

$P \quad$ CM of the moving platform

$Q_{S} \quad$ Supply flow rate.

$Q_{1} \quad$ Flow rate through the valve connection 1 .

$Q_{2} \quad$ Flow rate through the valve connection 2 .

$Q_{3} \quad$ Flow rate through the valve connection 3 .

$Q_{4} \quad$ Flow rate through the valve connection 4 .

$Q_{12} \quad$ Flow rate between the valve connection 1 and 2 . 


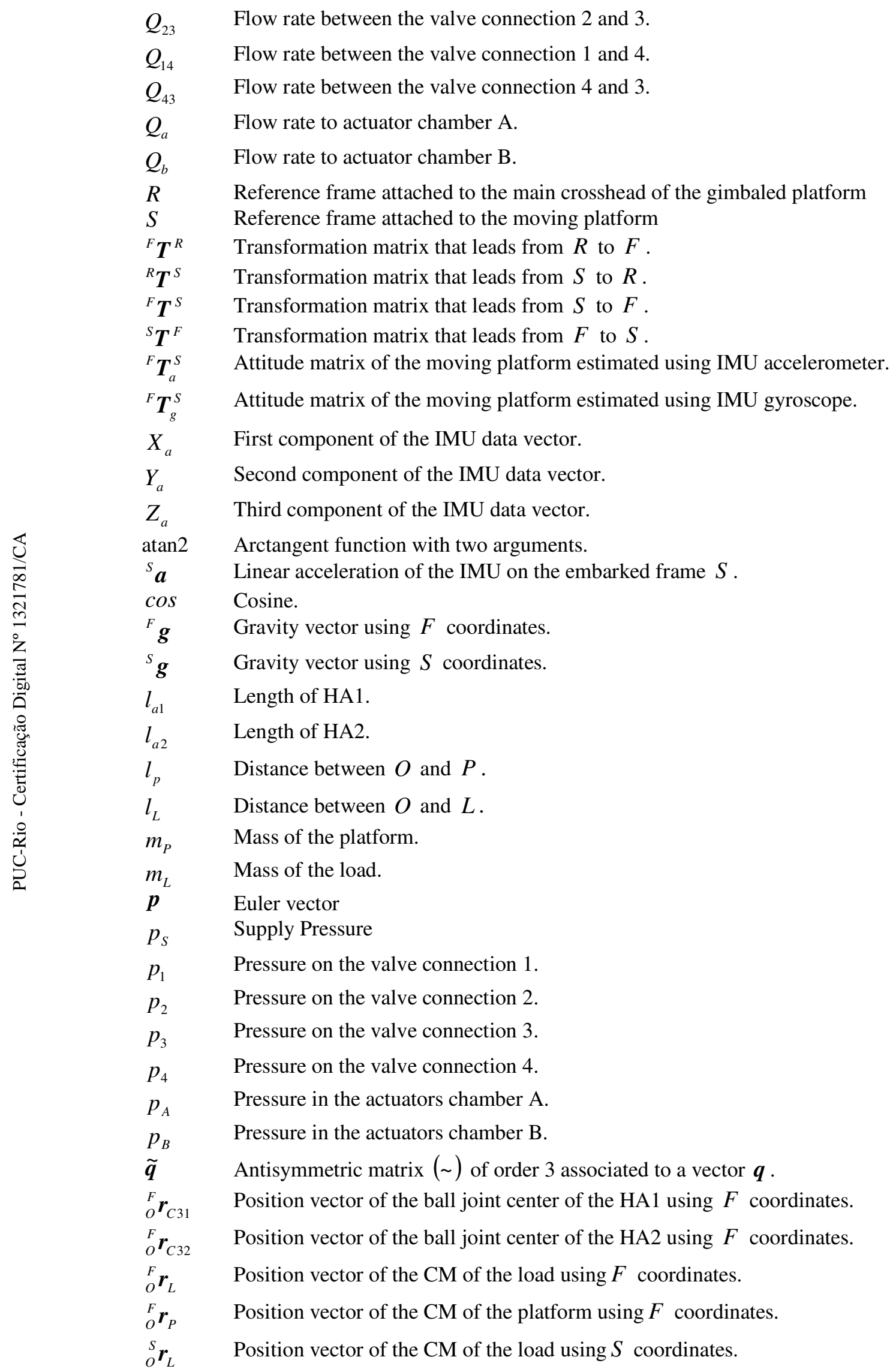


${ }_{O}^{S} \boldsymbol{r}_{P} \quad$ Position vector of the CM of the platform using $S$ coordinates.

${ }^{S}{ }_{S} \quad$ Data vector for IMU accelerometer using $S$ coordinates.

$\sin \quad$ Sine.

${ }^{S} \boldsymbol{v}_{P} \quad$ Linear velocity of the CM of the moving platform.

${ }^{S} \boldsymbol{v}_{L} \quad$ Linear velocity of the CM of the load.

${ }^{F} \boldsymbol{v}_{C_{31}} \quad$ Linear velocity of the ball joint center of the HA1 using $F$ coordinates.

${ }^{F} \boldsymbol{v}_{C_{32}} \quad$ Linear velocity of the ball joint center of the HA2 using $F$ coordinates.

$\alpha \quad$ Angle of rotation around the $\mathrm{x}^{\prime}$-axis of the gimbaled platform

$\beta \quad$ Angle of rotation around the $\mathrm{y}$-axis of the gimbaled platform

$\phi \quad$ Angle between the vector $\boldsymbol{p}$ and the $\mathrm{x}$-axis.

$\phi_{a} \quad \phi$ angle estimated using accelerometer data.

$\theta \quad$ Angle of rotation about $\boldsymbol{p}$.

$\theta_{a} \quad \theta$ angle estimated using accelerometer data.

$\theta_{V} \quad$ Angular position of the valve rotor.

${ }_{F}^{F} \omega_{R} \quad$ Angular velocity vector of the main crosshead using $F$ coordinates.

${ }_{F}^{R} \omega_{R} \quad$ Angular velocity vector of the main crosshead using $R$ coordinates.

${ }_{R}^{R} \omega_{S} \quad$ Angular velocity related to the main crosshead of the table using $R$ frame.

${ }_{R}^{S} \omega_{S} \quad$ Angular velocity related to the main crosshead of the table using $S$ frame.

${ }_{F}^{F} \omega_{S} \quad$ Angular velocity of the moving platform using $F$ coordinates.

${ }_{F}^{S} \omega_{S} \quad$ Angular velocity of the moving platform using $S$ coordinates. 


\section{List of Abbreviations}

AC Alternating current

CAD Computer aided design

$\mathrm{CF} \quad$ Complementary filter

$\mathrm{CM} \quad$ Center of mass

CVJ Constant velocity joint

DAQ Data acquisition system

DOF Degrees of freedom

EHA Electrohydraulic actuator

HA Hydraulic actuator

I2C Inter-Integrated Circuit

KF Kalman filter

PDHV Proportional Digital Hydraulic Valve

PID Proportional-integral-derivative

PPR Pulses per revolution

RF Reference frame

RTAE Real-time attitude estimation

TVC Thrust vector control 
"Success in space demands perfection. Many of the brilliant achievements made in this vast, austere environment seem almost miraculous. Behind each apparent miracle, however, stands the flawless performance of numerous highly complex systems. All are important." 


\section{1 Introduction}

Humanity has always been interested in the sky and motivated to explore it, extending the boundaries of our science. Since the last century, this challenge has created industries focused on advanced technologies, thus fostering the economic development of nations that invest in a space exploration program.

From the moment of launch, the space rocket has to have a steering system to guide it in a desired trajectory. Through this system, the propellant vector is deflected from the centerline of the rocket, thereby generating a moment about its center of mass to change the attitude of the spacecraft. This method to control the direction of the rocket is called thrust vector control (TVC).

In this Thesis, a low-cost TVC system is proposed in two configurations. First, in a classical design, a gimbaled nozzle is considered. As an alternative, a homokinetic tilting system is proposed. Through dynamic modeling, simulations show the effectiveness of both configurations and an experimental apparatus based on a fixed base robotic system validates the results.

\section{1 .} Fundamentals of TVC

In the TVC application, two hydraulic or two electromechanical actuators are usually used to swivel the thrust vector [1]. This system tilts the rocket nozzle to produce torque about its center of mass (CM) and change its attitude [2], as depicted in Fig. 1. 


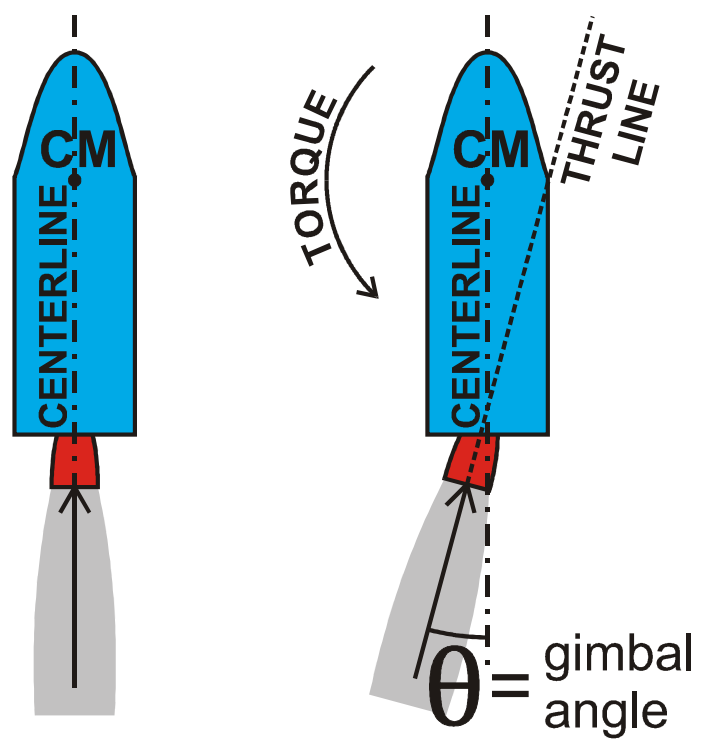

Figure 1.1: TVC method.

The electro-hydraulic actuators (EHAs) are the most widespread actuating system used in TVC. Aerospace applications require a high power density, high dynamic performance, robustness and overload capacity; leading the EHAs to be a common choice [3]. The TVC system must orient the nozzle on a desired trajectory for the vehicle and maintain the controllability during disturbances. Gust and shear wind are the main disturbances [4]. When using liquid-propellant, a gimbaled thrust engine is the typical option [5] to steer a vehicle along its path.

An EHA system is composed by a valve to modulate the fluid flow rate based on an input signal and a hydraulic cylinder that converts the flow rate into linear displacements [6].

There are two sources of energy, electric and hydraulic, for the EHA system. Electrical power is required to drive the valves and a peripheral system. The main source of hydraulic power is the fluid under pressure from the hydraulic power unit. For gimbal tests, a continuous source of hydraulic power is required, hence an electrohydraulic pump is used. But in several cases, a ground powered pump supplies the EHA system until the spacecraft take off. And afterwards, for upper stages and during a limited time, an accumulator provides the hydraulic power. During flight, the vehicle guidance system computes the deviation of the spacecraft from the desired trajectory. This deviation signal feds the TVC electronic system that controls the valve of EHAs. Traditionally, the feedback of the actual EHA position is measured through linear transducers [5]. 


\section{2.}

Literature review

This section reviews the related literature for this Thesis. First, an overview of TVC research is presented, including the topic of hydraulic control. Due to the similarities, this review also comprises some works about parallel robotics platforms. Furthermore, the attitude estimation references are introduced. In this Thesis it is proposed the replacement of the gimbal by a homokinetic joint, also called constant velocity joint $(\mathrm{CVJ})$. Therefore, this literature review also includes the CVJ and the universal joint literature with focus on kinematics.

Lazic and Ristanovic [7] proposed a test bench for a TVC system based on a fixed base robotic system. They presented an analysis of the kinematics and a control strategy for a gimbaled 2-DOF parallel platform with two EHAs. Two angular transducers provide the angular position of the gimbal, a DAQ system (Data Acquisition System) calculates the actuators position in real-time for the control algorithm, and two hydraulic servovalves receive the analog signal from the Proportional-Integral (PI) controller. Their experiments show a good correspondence with the numerical simulations and achieve a good position tracking. However, the validation of the numerical simulations considers the angular position only. A comparison using measured actuators force and pressure gives a more reliable analysis. In this Thesis, it is shown that in some condition, in spite of the increment of the actuator force, the position accuracy and the output of the controller does not change. Hence, load cells and pressure transducers are mounted in the experimental system to validate the actuators forces and chambers pressures results from the numerical simulations.

Wekerle et al. [4] presented the requirements of an actuation system for TVC systems. The working envelope of several TVC actuators from the literature is analyzed. A simplified and linearized model of a spacecraft as a rigid body is presented considering wind disturbances. During the development of this Thesis, the actuator requirements presented in [4] was used as a reference. In [8] Wekerle et al. presented a 2-dof mockup of a rocket motor nozzle with two EHAs. The actuators performance is identified in a mass-spring test bench. The TVC mockup presented in [8] uses two commercial servovalves; however in this Thesis lowcost valves are manufactured. The custom built valves reduce the cost and the 
complexity of the system, because the commercial servo valves need a super clean fluid and a sophisticated peripheral control system. Indeed, the EHA system presented in [8] is closer to the most widespread control system in the industry. The target of this Thesis is to propose a novel low-cost hydraulic system, but it needs a lot of development to achieve the robustness of the TVC presented in [8].

Ghosh et al. [9] developed a 2-dof parallel hydraulic actuated system for a heave-and-pitch motion simulator. Two low-cost commercial solenoid proportional valves control the hydraulic actuators. These types of valves have a dead-band and non-linear behavior [10]. The authors have considered different types of controllers, and the self-tuning fuzzy proportional-integral-derivative (PID) with bias control showed the best performance. The PID controller had the worst response [9]. The commercial proportional valves used in [9] have a deadband of $15 \%$ (usually, $5 \%-20 \%$ is common [36]). In this Thesis, the dead-band of the proposed valve is less than $0.5 \%$, hence a better performance of a linear controller is expected.

Traditionally, computed torque controllers add a dynamic compensation term to a PD controller. Le et al. [41] presented a 2-DOF parallel manipulator with a nonlinear PD computed torque controller. The controller combines the conventional computed torque with a self-gain tuning method using neural network. Numerical simulations showed the effectiveness of the proposed controller. In dynamic model used in the dynamic compensation, the position of the $\mathrm{CM}$ and the inertia of the links are $20 \%$ different from the dynamic model used in the numerical simulations. However, in practice other model divergences may play an important role, e.g. friction and stiffness.

Yang et al. [40] presented a PD control with gravity compensation for a 6DOF hydraulic parallel manipulator. The numerical and experimental result of the proposed controller indicates improvements on the stability and in the position accuracy. The large hydraulic manipulator with heavy payload suffers more influence of gravity than friction. The equations used to compute the gravitational term are the simplest within the dynamic modeling of the manipulator and the position feedback is the only necessary measurement. The simple implementation and relevant benefit makes the gravity compensation advisable for large hydraulic manipulators. 
Yu et al. [11] developed a novel rotary spool direct drive servovalve with axisymmetric flow. Experiments and computational fluid dynamics (CFD) simulations show the reduction of forces on the valve spool. The axisymmetric flow over the spool increases the pressures range, but the manufacturing complexity is also increased. In this Thesis, the influence of the forces on the spool is reduced using rolling bearings.

The angular position estimation of an IMU is a common topic of research, with the variations of the Kalman filter (KF) representing the most common option for sensor fusing between accelerometer and gyroscope data [12-14]. Accuracy improvements are achieved if the physical constraints are considered during the angular position estimation [15-16]. KF is able to model the noise of the IMU and obtain an optimal estimation of the attitude [17]. However, these stochastic approaches of the KF require a recursive calculation, making its realtime implementation unacceptable for low-cost hardware. A frequency-based approach, for example, a complementary filter (CF), requires a smaller computational cost [18]. In a $\mathrm{CF}$, the accelerometer data passes through a lowpass filter and the gyroscope data passes through a high-pass filter. The KF and $\mathrm{CF}$ have a maximum angular error of approximately less than $1^{\circ}$ for most of the applications [20-23].

Pennestrí et al. [24] conducted a kinematic analysis and a numerical simulation of a CVJ with geometric errors. The influence of the manufacturing errors is evaluated through a numerical example. The kinematics of a CVJ with straight tracks is proposed in a close form; however, it requires a forth degree polynomial equation to express the relation between the input and the output axis. In this Thesis, the relation between the axes is done through a transformation matrix obtained from a rotation around a vector, leading to a shorter kinematic modeling.

Most of the work about CVJ focuses on the powertrain issue. Kimata et al. analyzed the $\mathrm{CVJ}$ dynamics by taking into account the friction between moving parts [25]; furthermore, the results are validated with simulations and experiments [26]. Watson et al. [27] present a kinematics analysis of a Thompson Coupling. This mechanism is a double cardan with a spherical linkage that ensures the homokinetic condition [28]. 
On the way to construct this thesis, Cardozo and Weber [29] have introduced a 2-DOF mechanism similar to the gimbaled platform. In [30] numerical simulations have shown the efficacy of the same gimbaled platform of this Thesis. Still in [30], using an experimental apparatus the numerical results are validated. The homokinetic platform of this Thesis is analyzed numerically and experimentally in [31]. Many of prosperous results obtained in [29-31] are presented in this Thesis.

\section{3. Objective of this Thesis and summary of results}

This Thesis concerns the development of a 2-DOF parallel platform for variable orientation of a body in space. It starts with the design of a basic concept that is dynamically analyzed, simulated, constructed and finally validated. Several components were developed with the aim to have cheaper and reliable elements. They were by its own designed, built, analytically modeled, numerically simulated and validated. It should be able to replace a similar device which is used on TVC systems [1, 7, 29-31, 33]. Furthermore a homokinetic variation of the platform is proposed, that uses a CVJ with an IMU, instead of a gimbal with two angular transducers. Aiming cost reduction, a novel control valve called Proportional Digital Hydraulic Valve (PDHV) is used instead of commercial servovalves. This novel control valve reduces the system cost by more than one order of magnitude. The proposed controller is a simple proportional (P) controlled controller; however, it has an improved performance compared with the controller of Gosh et al. [9] because of the linear behavior of the PDHV.

In the homokinetic platform, a real-time attitude estimation (RTAE) system is used to measure the angular position. In this system, accelerometer and gyroscope sensor data fusion is performed using a CF considering the kinematic constrains of the CVJ. In addition, the CF is based on attitude matrices (usually called rotation matrix) instead of quaternions, Euler angles, or Cardan (TaitBryan) angles, which are the typical choices. The applicability of RTAE system is confirmed through the encoder data reference on the gimbaled platform. During this benchmarking, the constraint of the gimbal is considered and the average error reached $0.1^{\circ}$. 
In general, the experimental test bench verified the good position tracking and the lack of instabilities as indicated by the numerical simulation for the both platform configurations.

\section{4.}

Outline of this Thesis

This Thesis is divided in seven chapters and four appendices. The current chapter serves as an introduction and an overview of the work.

Chapter 2 presents the approach used in the dynamics, with the notation and the systematic.

Chapter 3 present the full development of both platform configurations. The kinematic description of the platforms is presented. Hence, in the dynamic modeling, an analysis is performed using the Newton-Euler formulation to obtain the equations of motion.

Chapter 4 investigates the hydraulic control system. The modeling of each element of the hydraulic system is shown. The concept of the novel control valve, PDHV, is presented. The model simplifications required for the numerical simulations are also discussed in this chapter.

Chapter 5 describes the details of data acquisition system (DAQ) developed specially for this Thesis and the control-loop. The IMU data fusion of RTAE system is explored.

Chapter 6 presents the simulation and experimental results. The method for validation of proposed system is investigated. The comparison between the results of both platform configurations is discussed. In addition, the influence of the load over the moving table is analyzed. This chapter finished illustrating a method to test novel control strategies.

Chapter 7 summarizes the conclusion remarks of the proposed system. Finally, suggestions for further work are offered.

Appendix A shows the preliminary test benches developed to identify the unknown parameters of the actuating system. The modeling of the hydraulics system was first validated in these test benches. It was also a first trial for the control system. 
Appendix B depict the electrohydraulic circuit developed for the experimental apparatus of this Thesis.

Appendix C presents the low-cost DAQ system developed instrumentation and control of the platform.

Appendix D gives some additional information about the rotation around a vector presented in the Chapter 2. 


\section{2 \\ Fundamentals of the kinematic and dynamic analysis}

The comprehension of the rigid body dynamics issues in the available literature presents some difficulties. The first barrier to the understanding is the notation: different authors have different notations. And there is also a lack of systematic in defining the different concepts. After a lifetime teaching, it led Prof. Hans Ingo Weber to develop an approach that overcomes these aspects. Unfortunately, his ideas are not published yet. Therefore, this chapter gives a brief introduction to his approach that is used in the dynamic modeling of the next chapter.

\section{1.}

\section{The rigid body motion}

A body in motion translates and rotates. There are 3-DOF associated to translation and another 3-DOF to rotation. The basic element in the study of translation is the position vector of a chosen point of the body to follow the motion. The components of this vector vary in time allowing establishing velocities and acceleration vectors of this point. If there is pure translation, all points have similar kinematics.

The first idea to describe the 3-DOF rotation would be defining angles, but this is not so easy at it seems. To keep the angles as independent coordinates, the rotations have to be around independent axes and in sequence, using axes from intermediate coordinate systems. These angles, if they are finite, do not obey the commutative property and therefore cannot be put together as a vector. The basic element in the study of the rotation is therefore not an angle, but the angular velocity. Its definition is crucial and it will be presented through a very special approach.

Other main difficulty in the study of rotation is related to the equations of motion. Using Newton's law for the center of mass of a body, one knows that an applied force on the body results in an acceleration that is parallel to the force 
since the mass is a scalar quantity. Using Euler's approach for the rotation on the other hand, shows that the direction of the applied torque is not parallel to angular accelerations since the inertia properties are due to the distribution of mass and instead of a scalar mass, they should be arranged an inertia tensor. The product of the matrix representing this tensor with the angular speed vector gives an angular momentum vector which normally is not parallel to the angular speed and may couple the equations representing the rotation in the different directions.

\section{2.}

\section{About the nomenclature}

The motions occur in a 3-dimensional space. Dynamics demand several reference frames (RF): calling the fixed $\mathrm{RF}$ as $F$, the body frame as $S$ and $Q, R$ as other frames. Each frame has its coordinates, e.g. $F(x, y, z)$. Vectors $(3 x 1)$ and matrices (3x3) will be written in bold: $\boldsymbol{r}$ (position of a point), $\boldsymbol{v}$ (velocity of a point), $\boldsymbol{a}$ (acceleration of a point), $\boldsymbol{\omega}$ (angular velocity of a body), $\boldsymbol{\xi}$ (angular acceleration of a body), $\boldsymbol{F}$ (force), $\boldsymbol{M}$ (moment of a force), $\boldsymbol{G}$ (linear momentum), $\boldsymbol{H}$ (angular momentum), I (inertia matrix), $\boldsymbol{T}$ (attitude matrix), $\tilde{\boldsymbol{\omega}}$ (the antisymmetric matrix associated to the vector $\boldsymbol{\omega}), \boldsymbol{E}$ (the identity matrix).

Some of these elements may be complemented with left/right sub/superscripts if necessary: ${ }_{A}^{S} \boldsymbol{r}_{P}$ position vector starting from point $A$ to point $P$ written in coordinates of $S ;{ }^{S} \boldsymbol{v}_{P}$ or ${ }_{O}^{S} \boldsymbol{v}_{P}$ (absolute) velocity coordinates in $S$ of point $P$ or referred to the fixed point $O$ (sometimes, if obvious this subscript is omitted); ${ }_{S}^{F} \boldsymbol{v}_{\text {relP }}$ is the relative velocity of point $P$ moving in $S$ written in coordinates of the fixed frame $F ;{ }_{Q}^{R} \omega_{R}$ is the angular velocity of frame $R$ relative to the frame $Q$ written in coordinates of $R$; if left subscript is omitted then it is the angular velocity referred to $F$ as ${ }^{R} \omega_{R}$ in coordinates of $F$.

Derivatives can be absolute and will be represented as $\frac{d \boldsymbol{r}}{d t}$, or local when the derivatives are obtained by using the components in the moving RF, as ${ }^{S} \dot{\boldsymbol{r}}$. Then we can see that $\frac{d^{F} \boldsymbol{r}}{d t}={ }^{F} \dot{\boldsymbol{r}}$, but $\frac{d^{S} \boldsymbol{r}}{d t} \neq \neq^{S} \dot{\boldsymbol{r}}$ since it still needs to include the part 
due to the rotation of the RF. Then, ${ }^{S} \dot{\boldsymbol{r}}={ }_{S}^{S} \boldsymbol{v}_{\text {rel }}$ but need to take care with ${ }_{S}^{F} \boldsymbol{v}_{r e l}={ }^{F} \boldsymbol{T}{ }^{S S} \dot{\boldsymbol{r}}={ }^{F} \boldsymbol{T}{ }^{S}{ }_{S}^{S} \boldsymbol{v}_{r e l} \neq{ }^{F} \dot{\boldsymbol{r}}={ }^{F} \boldsymbol{v}$.

\section{3.}

\section{Vector product and the tilde matrix}

When multiplying two vectors to obtain a third vector, it is possible to associate the first vector $(3 x 1)$ with an antisymmetric matrix $(3 \times 3$, which has three independent nonzero elements). The rules that should be obeyed are that the result is orthogonal to both vectors and that the magnitude is the product of the magnitudes of both vectors times the sinus of the angle between them. To simplify the denominations, this is called the tilde matrix associated to the vector. In equations:

$$
\boldsymbol{r}=\left[\begin{array}{c}
r_{x} \\
r_{y} \\
r_{z}
\end{array}\right] \Rightarrow \tilde{\boldsymbol{r}}=\left[\begin{array}{ccc}
0 & -r_{z} & r_{y} \\
r_{z} & 0 & -r_{x} \\
-r_{y} & r_{x} & 0
\end{array}\right] ; \boldsymbol{t}=\tilde{\boldsymbol{r}} \boldsymbol{s}=\left[\begin{array}{l}
r_{y} s_{z}-r_{z} s_{y} \\
r_{z} s_{x}-r_{x} s_{z} \\
r_{x} s_{y}-r_{y} s_{x}
\end{array}\right]
$$

This notation has several advantages in Mechanics since, for example, the vector triple product $\boldsymbol{\omega} x(\boldsymbol{\omega} x \mathbf{r})$ can be simplified to $\tilde{\boldsymbol{\omega}}^{2} \mathbf{r}$.

\section{4.}

The attitude matrix

The rotation of a body results in a new position. One may imagine one of the points of the body chosen for the description of translation, then, in general, there is a 3 DOF rotation around this point. Knowing the geometry of the body, its position can be represented in the fixed reference frame using the attitude matrix. In modern times, due to all numerical possibilities given by computers, a technique like visualization has become a standard method to handle with the dynamics of systems.

If the body geometry is known in body coordinates $S$, then the attitude matrix transforms this coordinates to $F$. The following nomenclature is propose:

$$
{ }^{F} \boldsymbol{r}_{P}={ }^{F} \boldsymbol{r}_{A}+{ }_{A}^{F} \boldsymbol{r}_{P}={ }^{F} \boldsymbol{r}_{A}+{ }^{F} \boldsymbol{T}^{S}{ }_{A}^{S} \boldsymbol{r}_{P}
$$


where $O$ is the fixed origin of the reference frame $F$, omitted in the left subscript of $r, A$ is the point of the body that contains the information on the translation given by ${ }^{F} \boldsymbol{r}_{A}(t)$ and ${ }_{A}^{S} \boldsymbol{r}_{P}$ represents the geometry of the body in $S$ since $P$ is a generic point of this body. The attitude matrix ${ }^{F} \boldsymbol{T}^{S}$ allows drawing the body in $F$. Translation has no effect on this matrix and it will not be considered further. The problem will consist of:

$$
{ }^{F} \boldsymbol{r}={ }^{F} \boldsymbol{T}^{S S} \boldsymbol{r} .
$$

Matrix $\boldsymbol{T}$ received a good amount of names along the years starting as director cosine matrix, as coordinate transformation matrix and also as rotation matrix, depending on the way it is obtained. This approach suggests to rename it in a modern way as attitude matrix.

The action of this matrix multiplying a vector will be examined with care: the length of the vector must be the same and the direction stays also the same along the transformation. The inverse problem is the result of changing the superscripts: ${ }^{F} \boldsymbol{r}={ }^{F} \boldsymbol{T}^{S S} \boldsymbol{r}$ to ${ }^{S} \boldsymbol{r}={ }^{S} \boldsymbol{T}^{F F} \boldsymbol{r}$. This will result in ${ }^{F} \boldsymbol{r}={ }^{F} \boldsymbol{T}^{S S}{ }^{S}{ }^{F F} \boldsymbol{r}$ meaning that ${ }^{F} \boldsymbol{T}^{S S} \boldsymbol{T}^{F}=\boldsymbol{E}$, i.e. the identity matrix. If it is consider ${ }^{S} \boldsymbol{r}$ as the unitary basis vectors of the reference frame $S$, then from inspection that the columns of ${ }^{F} \boldsymbol{T}^{S}$ are the representation in $F$ of the basis vectors of $S$. Moreover, the vector-multiply of a vector in the $x$ direction with a vector in the $y$ direction it should result in a vector in the $z$ direction, in any of the representations $S$ or $F$, since our coordinate systems are right handed. One concludes that the attitude matrix is an orthogonal matrix.

Another comment is that if there are 3-DOF, the 9 elements of this matrix must be function only of three distinct variables. But if these are the best variables to solve the problem is another question.

If the body is rigid all vectors between points of the body ${ }^{S} \boldsymbol{r}$ have constant components, then the only time dependency in this transformation is contained in ${ }^{F} \boldsymbol{T}^{S}$. The time variation of the attitude matrix must give us information about the angular velocity of the body. Because it is an orthogonal matrix, there will be again an important property for its derivative when multiplied with the transposed. The orthogonality implies in 


$$
{ }^{F} \boldsymbol{T}^{\text {S S }} \boldsymbol{T}^{F}=\boldsymbol{E} \Rightarrow\left[\begin{array}{lll}
t_{11} & t_{12} & t_{13} \\
t_{21} & t_{22} & t_{23} \\
t_{31} & t_{32} & t_{33}
\end{array}\right]\left[\begin{array}{ccc}
t_{11} & t_{21} & t_{31} \\
t_{12} & t_{22} & t_{32} \\
t_{13} & t_{23} & t_{33}
\end{array}\right]=\left[\begin{array}{lll}
1 & 0 & 0 \\
0 & 1 & 0 \\
0 & 0 & 1
\end{array}\right] .
$$

As a consequence, the differentiation in time leads to:

$$
\begin{aligned}
& t_{11} t_{11}+t_{12} t_{12}+t_{13} t_{13}=1 \Rightarrow \dot{t}_{11} t_{11}+\dot{t}_{12} t_{12}+\dot{t}_{13} t_{13}=0 \\
& t_{21} t_{11}+t_{22} t_{12}+t_{23} t_{13}=0 \Rightarrow \dot{t}_{21} t_{11}+\dot{t}_{22} t_{12}+\dot{t}_{23} t_{13}=-t_{21} \dot{t}_{11}-t_{22} \dot{t}_{12}-t_{23} \dot{t}_{13}
\end{aligned}
$$

One concludes that ${ }^{F} \dot{\boldsymbol{T}}^{S S} \boldsymbol{T}^{F}$ is anti-symmetric, which is basic to define angular velocity.

With focus on the rotation of the position vector that connects 2 points of a rigid body, say points $A$ and $P$, it can develop from its derivative that,

$$
\frac{d_{A}^{F} \boldsymbol{r}_{P}}{d t}={ }_{A}^{F} \boldsymbol{v}_{P}=\frac{d}{d t}\left({ }^{F} \boldsymbol{T}^{S}{ }_{A}^{S} \boldsymbol{r}_{P}\right)={ }^{F} \dot{\boldsymbol{T}}^{S S}{ }_{A}^{S} \boldsymbol{r}_{P}+\underbrace{{ }^{F} \boldsymbol{T}^{S S}{ }_{A}^{S} \dot{\boldsymbol{r}}_{P}}_{=0}={ }^{F} \dot{\boldsymbol{T}}^{S S} \boldsymbol{T}^{F}{ }_{A}^{F} \boldsymbol{r}_{P}={ }_{F}^{F} \widetilde{\boldsymbol{\omega}}_{S A}^{F} \boldsymbol{r}_{P} .
$$

The time derivative of the constant components of the vector in $S$ is zero and the equation tells that the time derivative of a constant vector in a rotating system is given by the cross product of the angular velocity of this system with the vector. This result can also be written as ${ }_{A} \boldsymbol{v}_{P}=\widetilde{\boldsymbol{\omega}}_{S A} \boldsymbol{r}_{P}$, since a vector equation may be solved in any reference frame.

\section{5 .}

\section{Angular velocity definition}

The definition of angular velocity using the tilde representation is given by, ${ }_{F}^{F} \widetilde{\boldsymbol{\omega}}_{S}={ }^{F} \dot{\boldsymbol{T}}^{S S} \boldsymbol{T}^{F}$.

Changing the representation to $S$ implies in:

$$
{ }_{F}^{S} \widetilde{\boldsymbol{\omega}}_{S}={ }^{S} \boldsymbol{T}^{F F}{ }_{F}^{F} \widetilde{\boldsymbol{\omega}}_{S}{ }^{F} \boldsymbol{T}^{S}={ }^{S} \boldsymbol{T}^{F F} \dot{\boldsymbol{T}}^{S} \text {. }
$$

Since finite angles do not form a vector, but the angular velocity is a vector one central problem of space dynamics is how to proceed to construct the attitude matrix in an elegant way. There are two complete different approaches for that, one dividing the motion in sequential independent rotations and the other discovering a direction and using a single rotation around it. 


\section{6.}

\section{Attitude matrix obtained by sequential rotations}

In this case, having in mind that ${ }^{F} \boldsymbol{r}={ }^{F} \boldsymbol{T}^{S S} \boldsymbol{r}$ and looking for the attitude matrix of a planar rotation about one of the coordinate axis, it can be obtained by inspection. It is also possible to break the motion in parts and can choose 3 partial elementary rotations resulting in intermediate RF $Q$ and $R$. This means that,

$$
{ }^{F} \boldsymbol{r}={ }^{F} \boldsymbol{T}^{Q Q} \boldsymbol{r}={ }^{F} \boldsymbol{T}^{Q Q} \boldsymbol{T}^{R R} \boldsymbol{r}={ }^{F} \boldsymbol{T}^{Q Q} \boldsymbol{T}^{R R} \boldsymbol{T}^{S S} \boldsymbol{r}={ }^{F} \boldsymbol{T}^{S S} \boldsymbol{r},
$$

and the final attitude matrix is a product of the partial rotation matrices.

On the other hand when defining the angular velocity of the body in terms of these sequential rotations:

$$
\begin{aligned}
& { }_{F}^{F} \tilde{\boldsymbol{\omega}}_{Q}={ }^{F} \dot{\boldsymbol{T}}^{Q Q} \boldsymbol{T}^{F} \\
& { }_{Q}^{Q} \tilde{\boldsymbol{\omega}}_{R}={ }^{Q} \dot{\boldsymbol{T}}^{R Q} \boldsymbol{T}^{R} \\
& { }_{F}^{F} \tilde{\boldsymbol{\omega}}_{R}={ }^{F} \dot{\boldsymbol{T}}^{R R} \boldsymbol{T}^{F}=\left({ }^{F} \dot{\boldsymbol{T}}^{Q Q}{ }^{Q} \boldsymbol{T}^{R}+{ }^{F} \boldsymbol{T}^{Q Q}{ }^{\boldsymbol{T}^{R}}\right)^{R} \boldsymbol{T}^{Q Q} \boldsymbol{T}^{F}={ }^{F} \dot{\boldsymbol{T}}^{Q Q} \boldsymbol{T}^{F}+{ }^{F} \boldsymbol{T}^{Q Q}{ }^{Q} \dot{\boldsymbol{T}}^{R R} \boldsymbol{T}^{Q Q} \boldsymbol{T}^{F}= \\
& ={ }_{F}^{F} \tilde{\boldsymbol{\omega}}_{Q}+{ }^{F} \boldsymbol{T}^{Q Q}{ }_{Q}^{Q} \tilde{\boldsymbol{\omega}}_{R}{ }^{Q} \boldsymbol{T}^{F}={ }_{F}^{F} \tilde{\boldsymbol{\omega}}_{Q}+{ }_{Q}^{F} \tilde{\boldsymbol{\omega}}_{R}
\end{aligned}
$$

This shows that with sequential rotations, while the attitude matrices are multiplied, the angular velocities are summed. The sequence of rotations frequently is defined obeying the restrictions of the supports that allow and restrict the motion, but they also can be chosen arbitrarily when a body is moving in space. If the angles are finite (nonlinear condition) once established, the sequence must be kept the same. Since each of the rotation is done around a coordinate axis, this axis is kept the same in both frames. The rotations must be independent, therefore two situations can happen: after two rotations the third rotation can be done around the third axis or around the first axis in its displaced position. Exemplifying the first group known as Cardan (or Thomson-Tait) angles, it is suggested the following diagram:

$$
\underset{(x, y, z)}{\boldsymbol{F}} \stackrel{\alpha(x)}{\longrightarrow} \bigcup_{\left(x, y^{\prime}, z^{\prime}\right)} \stackrel{\beta\left(y^{\prime}\right)}{\longrightarrow} \boldsymbol{R}_{\left(x^{\prime \prime}, y^{\prime}, z^{\prime \prime}\right)} \stackrel{\gamma\left(z^{\prime \prime}\right)}{\longrightarrow} \boldsymbol{S}_{\left(x^{\prime \prime \prime}, y^{\prime \prime \prime}, z^{\prime \prime}\right)}
$$

where:

$$
{ }^{F} \boldsymbol{T}^{Q}=\left[\begin{array}{ccc}
1 & 0 & 0 \\
0 & \cos \alpha & -\sin \alpha \\
0 & \sin \alpha & \cos \alpha
\end{array}\right]
$$




$$
\begin{aligned}
{ }^{Q} \boldsymbol{T}^{R} & =\left[\begin{array}{ccc}
\cos \beta & 0 & \sin \beta \\
0 & 1 & 0 \\
-\sin \beta & 0 & \cos \beta
\end{array}\right], \\
{ }^{R} \boldsymbol{T}^{S} & =\left[\begin{array}{ccc}
\cos \gamma & -\sin \gamma & 0 \\
\sin \gamma & \cos \gamma & 0 \\
0 & 0 & 1
\end{array}\right] .
\end{aligned}
$$

And, for example in $F$ the angular velocity ${ }_{F}^{F} \omega_{S}={ }_{F}^{F} \omega_{Q}+{ }_{Q}^{F} \omega_{R}+{ }_{R}^{F} \omega_{S} \quad$ is given by,

$$
{ }_{F}^{F} \boldsymbol{\omega}_{S}=\left[\begin{array}{c}
\dot{\alpha} \\
0 \\
0
\end{array}\right]+{ }^{F} \boldsymbol{T}^{Q}\left[\begin{array}{c}
0 \\
\dot{\beta} \\
0
\end{array}\right]+{ }^{F} \boldsymbol{T}^{R}\left[\begin{array}{l}
0 \\
0 \\
\dot{\gamma}
\end{array}\right] .
$$

And, in this case, ${ }_{F}^{F} \omega_{Q}={ }_{F}^{Q} \omega_{Q},{ }_{Q}^{Q} \omega_{R}={ }_{Q}^{R} \omega_{R},{ }_{R}^{R} \omega_{S}={ }_{R}^{S} \omega_{S}$.

\section{7. \\ Attitude matrix as rotation matrix}

Using the alternative procedure, considering again, ${ }^{F} \boldsymbol{r}={ }^{F} \boldsymbol{T}^{S S} \boldsymbol{r}$. Imagine $S$ moving from an initial position where it is coincident with $F$ to a later position anywhere in space (only rotations need to be taken in account). The product with the matrix will rotate all the possible unitary vector except one, which is the eigenvector of the matrix and this is the direction around which the rotation takes place. This special vector is called invariant vector $\boldsymbol{p}$. An orthogonal matrix have a unitary eigenvalue resulting in the equation $\boldsymbol{p}={ }^{F} \boldsymbol{T}^{S} \boldsymbol{p}$ or using the proposed notation, ${ }^{F} \boldsymbol{p}={ }^{S} \boldsymbol{p}=\boldsymbol{p}$. Geometrically speaking, the generic body vector $\boldsymbol{r}$ leaving from the origin of the RF moves over a conical surface according to the angle between $\boldsymbol{p}$ and $\boldsymbol{r}$. Fig. 2.1 gives some insight in what occurs. All these considerations give origin to the name "rotation matrix" to what is called attitude matrix. The explained procedure is outside of any time scaling. 


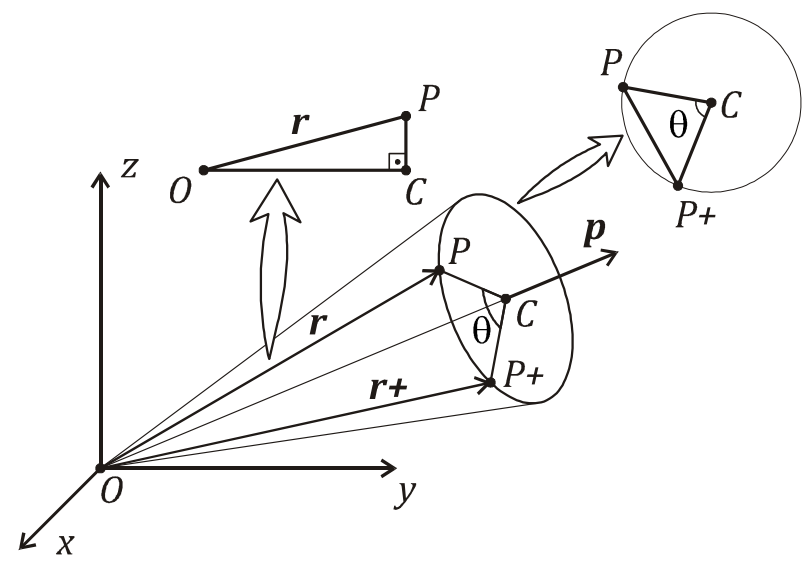

Figure 2.1: Rotation around the Euler axis $\boldsymbol{p}$.

From Fig. 2.1 it is be able to relate the attitude matrix to the so called Euler parameters, $\boldsymbol{p}$ (unit vector) and $\theta$. Vector $\boldsymbol{r}$ connects the origin $O$ with point $P$ and rotate by an angle $\theta$ around axis $\boldsymbol{p}$ to occupy a new position $\boldsymbol{r}+$. It leads to the vector equation (explained in the Appendix D),

$$
\boldsymbol{r}+={ }_{O} \boldsymbol{r}_{P+}={ }_{O} \boldsymbol{r}_{P}+{ }_{P} \boldsymbol{r}_{C}+{ }_{C} \boldsymbol{r}_{P+}=\boldsymbol{r}+(1-\cos \theta) \tilde{\boldsymbol{p}}^{2} \boldsymbol{r}+\sin \theta \tilde{\boldsymbol{p}} \boldsymbol{r} .
$$

By the identification of the initial position vector ${ }_{o} \boldsymbol{r}_{P}$ as ${ }^{S} \boldsymbol{r}$ and the final position $\boldsymbol{r}+$ as ${ }^{F} \boldsymbol{r}$ it concludes from (2.3) that,

$$
{ }^{F} \boldsymbol{T}^{S}=\boldsymbol{E}+(1-\cos \theta) \tilde{\boldsymbol{p}}^{2}+\tilde{\boldsymbol{p}} \sin \theta .
$$

Comparing the sequential and the rotation approaches, in the first case three sequential angles are used while in the present case there are used an angle and a direction. The dependence of the angular velocity with the Euler parameters is given substituting Eq. (2.18) and its time derivative in Eq. (2.7), resulting after a long calculation:

$$
{ }_{F}^{F} \boldsymbol{\omega}_{S}=\dot{\theta}^{F} \boldsymbol{p}+(1-\cos \theta)^{F} \tilde{\boldsymbol{p}}^{F} \dot{\boldsymbol{p}}+\sin \theta^{F} \dot{\boldsymbol{p}},
$$

To write this expression in body coordinates a curious situation must be observed. Since the Euler vector is invariant to the rotation ${ }^{F} \boldsymbol{p}={ }^{S} \boldsymbol{p}$ and the derivative of these coordinates lead to ${ }^{F} \dot{\boldsymbol{p}}={ }^{S} \dot{\boldsymbol{p}}$, i.e. the absolute derivative of the coordinates are equal to the local derivatives in the body frame $S$. Using Eq. (2.18) to transform Eq. (2.19) to $S$, then,

$$
{ }_{F}^{S} \boldsymbol{\omega}_{S}=\dot{\boldsymbol{\theta}} \boldsymbol{p}-(1-\cos \theta) \tilde{\boldsymbol{p}}^{S} \dot{\boldsymbol{p}}+\sin \theta^{S} \dot{\boldsymbol{p}} .
$$


The next chapter shows that Eq. (2.18) gives a simple relation for the kinematics of the homokinetic platform. And the systematic of the Section 2.6 has a practical application in the gimbaled platform. 


\section{3 \\ Platform development}

Since Word War II, when the Nazi Germany developed the V-2 missile, a lot of research has been done on rocket science [32]. The rocket steering device has an important role in this field of research. This Thesis proposes a 2-DOF parallel platform that is analogous to the gimbaled TVC system responsible for the steer of a class of spacecrafts.

This chapter presents the modeling of two platform configurations. Following what is more traditional in liquid-propellant TVC systems, a gimbaled platform is considered, as seen in [1, 5, 7, 29-31, 33]. As an alternative to this conventional design, the second part of this chapter proposes a homokinetic connection between the base and the moving table. Figure 3.1 shows an overview of the gimbaled platform with the hydraulic power unit, the electric drives and the instrumentation system.

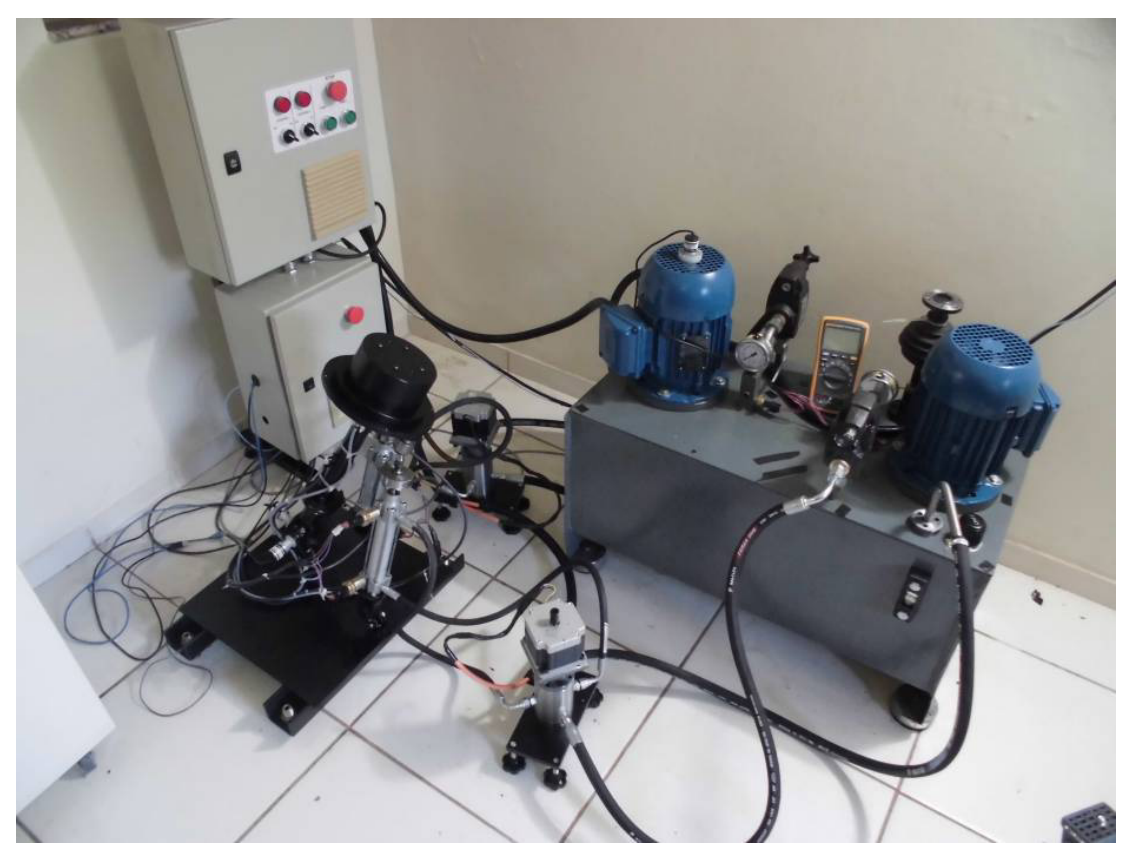

Figure 3.1: Platform overview with the peripheral system. 
For each platform configuration the analysis starts with a kinematic description. Thereafter, the equations of motion are obtained through the dynamic modeling. The Newton-Euler formulation is used because the reaction forces on the base are analyzed in the result section. Furthermore, the constraints forces were used during the structural calculus of the system.

\section{1.}

\section{Gimbaled platform}

The gimbaled platform brings the mainstream of the liquid-propellant TVC technology. This section starts with a full kinematic analysis following the systematic presented in the last chapter. Thereafter, using the Newton-Euler formulation, the dynamic model with the equation of movement is obtained.

\subsection{1.}

\section{Kinematics}

The gimbaled platform is designed with two EHA mounted around an universal joint, thus forming a 2-DOF parallel manipulator. Figure 3.2 shows this universal joint with two rotary encoders to measure the angular displacement of the crosshead.

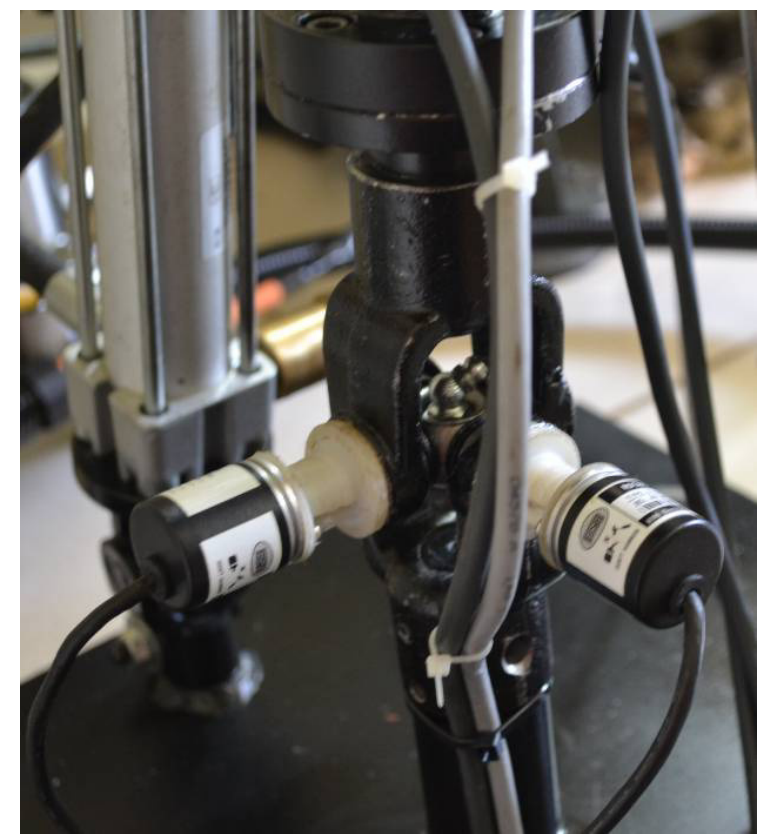

Figure 3.2: Universal joint with encoders. 
Figure 3.3 shows the concept of the gimbaled platform with a load over the moving table. One extremity of the universal joint is attached to the fixed base and the other one to the moving table. The EHAs are positioned with a geometry such that the necessary working area of the moving table is achieved.

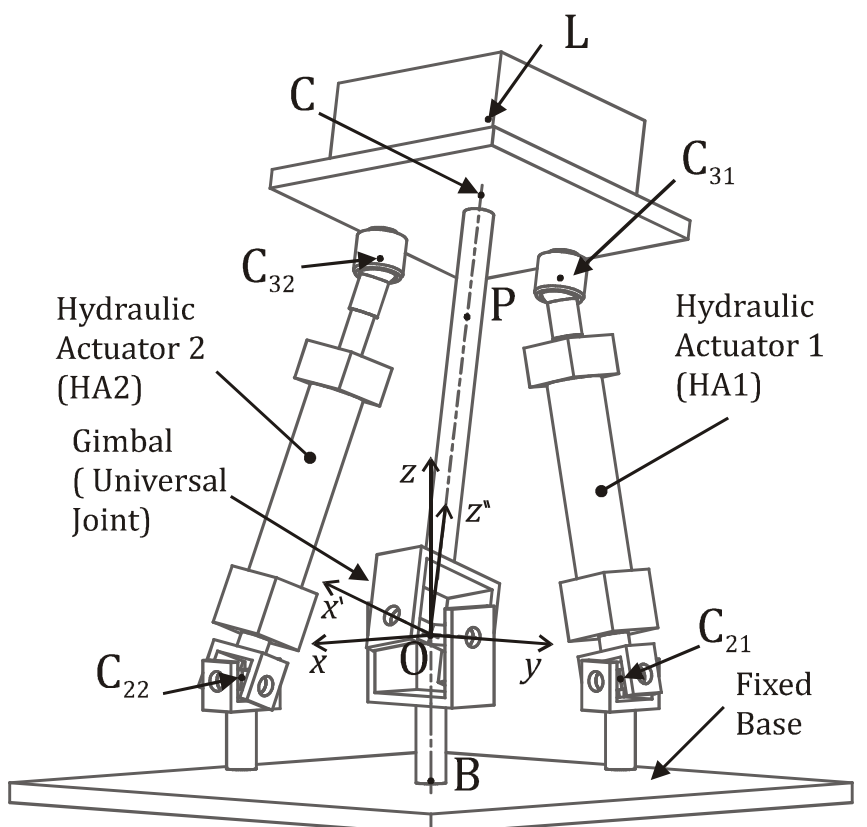

Figure 3.3: Gimbaled platform kinematic scheme.

For simpler kinematics analysis, the actuators are equal and its mounting points are symmetric around the z-axis [34]. The point $B$ is the center of the fixed base and $O$ is the center of the crosshead of the gimbal. A smaller universal joint connects the hydraulic actuators to the base and a ball joint connects its rod to the platform. Points $C_{31}$ and $C_{32}$ are the centers of the actuators ball joints. $C_{21}$ and $C_{22}$ are the centers of the actuators universal joint crossheads. $P$ and $L$ are the centers of mass of the moving platform and of the load, respectively. $C$ is the center of the top surface of the moving platform.

In order to analyze the kinematics, three references frames are defined, $F$ fixed to the base, $R$ attached to the main crosshead and $S$ attached to the moving platform. The rotation between the frames are summarized by the following indication, 


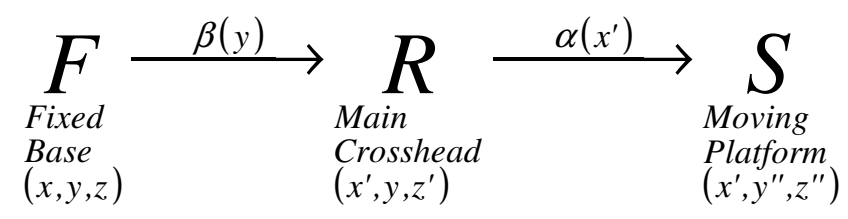

$\beta$ is the angle of rotation around the $\mathrm{y}$-axis and $\alpha$ is around the $\mathrm{x}^{\prime}$-axis. Both angles are measured using angular transducers. The transformation (or rotation, or attitude) matrix ${ }^{F} \boldsymbol{T}^{R}$ leads from $R$ to $F$,

$$
{ }^{F} \boldsymbol{T}^{R}=\left[\begin{array}{ccc}
\cos \beta & 0 & \sin \beta \\
0 & 1 & 0 \\
-\sin \beta & 0 & \cos \beta
\end{array}\right],
$$

and ${ }^{R} \boldsymbol{T}^{S}$ leads from $S$ to $R$,

$$
{ }^{R} \boldsymbol{T}^{S}=\left[\begin{array}{ccc}
1 & 0 & 0 \\
0 & \cos \alpha & -\sin \alpha \\
0 & \sin \alpha & \cos \alpha
\end{array}\right] .
$$

The angular velocity vector of the main crosshead using $R$ or $F$ coordinates is given by,

$$
{ }_{F}^{F} \boldsymbol{\omega}_{R}={ }_{F}^{R} \boldsymbol{\omega}_{R}=\left[\begin{array}{c}
0 \\
\dot{\beta} \\
0
\end{array}\right] .
$$

The vector of angular velocity related to the main crosshead of the moving platform, using $R$ or $S$ coordinates, is shown by,

$$
{ }_{R}^{R} \omega_{S}={ }_{R}^{S} \omega_{S}=\left[\begin{array}{c}
\dot{\alpha} \\
0 \\
0
\end{array}\right] .
$$

Hence, Eq.(3.5) gives the angular velocity of the moving platform using $S$ coordinates,

${ }_{F}^{S} \omega_{S}={ }^{S} T_{F}^{R}{ }_{F} \omega_{R}+{ }_{R}^{S} \omega_{S}$.

The position of the center of mass of the platform and the load it is carrying, using $S$ coordinates, are given by:

${ }_{O}^{S} \boldsymbol{r}_{P}=\left[\begin{array}{lll}0 & 0 & l_{p}\end{array}\right]^{T},{ }_{O}^{S} \boldsymbol{r}_{L}=\left[\begin{array}{lll}0 & 0 & l_{L}\end{array}\right]^{T}$.

In the fixed frame $F$, it results in

$$
{ }_{O}^{F} \boldsymbol{r}_{P}={ }^{F} \boldsymbol{T}^{S}{ }_{O}^{S} \boldsymbol{r}_{P},{ }_{O}^{F} \boldsymbol{r}_{L}={ }^{F} \boldsymbol{T}^{S}{ }_{O}^{S} \boldsymbol{r}_{L},
$$

where, ${ }^{F} \boldsymbol{T}^{S}={ }^{F} \boldsymbol{T}^{R R} \boldsymbol{T}^{S}$. 
Equation (3.10) describes the velocity of the center of mass of the moving platform, and Eq. (3.11) describes the velocity of the load,

$$
{ }^{S} \boldsymbol{v}_{P}={ }_{F}^{S} \tilde{\boldsymbol{\omega}}_{S}{ }_{O}^{S} \boldsymbol{r}_{P},{ }^{S} \boldsymbol{v}_{L}={ }_{F}^{S} \tilde{\boldsymbol{\omega}}_{S}{ }_{O}^{S} \boldsymbol{r}_{L} .
$$

Here, the special notation presented in the last chapter is used for an antisymmetric matrix $(\sim)$ of order 3 . Because the vector angular velocity is $(3 \times 1)$ and the $(3 \times 3)$ antisymmetric matrix has 3 different elements, the matrix ${ }_{F}^{S} \widetilde{\boldsymbol{\omega}}_{S}$ is given by,

$$
{ }_{F}^{S} \tilde{\boldsymbol{\omega}}_{S}=\left[\begin{array}{ccc}
0 & -\omega_{z} & \omega_{y} \\
\omega_{z} & 0 & -\omega_{x} \\
-\omega_{y} & \omega_{x} & 0
\end{array}\right] .
$$

The length of the hydraulic actuators 1 (HA1) is given by,

$$
l_{a 1}=\left|{ }_{C_{21}}^{F} \boldsymbol{r}_{C_{31}}\right|=\left|{ }_{O}^{F} \boldsymbol{r}_{C_{31}}-{ }_{O}^{F} \boldsymbol{r}_{C_{21}}\right|=\left|{ }^{F} \boldsymbol{T}^{S}{ }_{O}^{S} \boldsymbol{r}_{C_{31}}-{ }_{O}^{F} \boldsymbol{r}_{C_{21}}\right| \text {. }
$$

And in a similar manner, the length of the hydraulic actuators 2 (HA2) is,

$$
l_{a 2}=\left|{ }_{C_{22}}^{F} \boldsymbol{r}_{C_{32}}\right|=\left|{ }_{O}^{F} \boldsymbol{r}_{C_{32}}-{ }_{O}^{F} \boldsymbol{r}_{C_{22}}\right|=\left|{ }^{F} \boldsymbol{T}^{S}{ }_{O}^{S} \boldsymbol{r}_{C_{32}}-{ }_{O}^{F} \boldsymbol{r}_{C_{22}}\right| \text {. }
$$

In Eqs. (3.13) and (3.14), ${ }_{o}^{S} \boldsymbol{r}_{C_{31}}$ and ${ }_{o}^{S} \boldsymbol{r}_{C_{32}}$ are position vectors of the ball joint centers $C_{31}$ and $C_{32}$, respectively, using RF attached to the moving platform. ${ }_{0}^{F} \boldsymbol{r}_{C_{21}}$ and ${ }_{O}^{F} \boldsymbol{r}_{C_{22}}$ are the position vectors of the universal joint centers $C_{21}$ and $C_{22} C_{32}$, respectively, using the fixed RF. They are well known, because they are obtained through the CAD drawing of the platform.

The longitudinal displacement speed of each EHA is given by the projection of the ball joint center velocity on a unit vector parallel to the rod using the dot (inner) product:

$$
i_{a 1}={ }^{F} \boldsymbol{v}_{C_{31}}^{T} \frac{{ }_{C 21}^{F} \boldsymbol{r}_{C_{31}}}{\left|{ }_{C_{21}} \boldsymbol{r}_{C_{31}}\right|}, i_{a 2}={ }^{F} \boldsymbol{v}_{C_{32}}^{T} \frac{{ }^{C_{22}} \boldsymbol{r}_{C_{32}}}{\left|{ }_{C_{22}}{ }^{F} \boldsymbol{r}_{C_{32}}\right|},
$$

where the velocities of the $C_{31}$ and $C_{32}$ points are given by

$$
{ }^{F} \boldsymbol{v}_{C_{31}}={ }_{F}^{F} \tilde{\boldsymbol{\omega}}_{S O}{ }_{O}^{F} \boldsymbol{r}_{C_{31}},{ }^{F} \boldsymbol{v}_{C_{32}}={ }_{F}^{F} \tilde{\boldsymbol{\omega}}_{S O}{ }^{F} \boldsymbol{r}_{C_{32}} .
$$

The speed of the actuators is used in the numerical simulations to compute the friction force on the EHAs. The linear and angular velocities of the platform are used to calculate the linear and angular momentum of the moving platform. 
And then, using the Newton-Euler formulation, in the next section it is shown how to calculate the equation of motion of the system.

\subsection{2. \\ Dynamics}

Due to the symmetries of the moving platform and the load, theirs inertia matrix about its center of mass are given by,

$$
{ }^{S} \boldsymbol{I}_{P}=\left[\begin{array}{ccc}
I_{1 P} & 0 & 0 \\
0 & I_{1 P} & 0 \\
0 & 0 & I_{3 P}
\end{array}\right],{ }^{S} \boldsymbol{I}_{L}=\left[\begin{array}{ccc}
I_{1 L} & 0 & 0 \\
0 & I_{1 L} & 0 \\
0 & 0 & I_{3 L}
\end{array}\right]
$$

The vectors of angular momentum of the moving platform and the load using $S$ frame about its center of mass are introduced by,

$$
{ }^{S} \boldsymbol{H}_{P}={ }^{S} \boldsymbol{I}_{P}{ }_{F}^{S} \boldsymbol{\omega}_{S},{ }^{S} \boldsymbol{H}_{L}={ }^{S} \boldsymbol{I}_{L F}{ }_{F}^{S} \boldsymbol{\omega}_{S}
$$

The linear momentum vector of the platform and the load are given by Eq.(3.23) and (3.24) using $S$ frame,

$$
{ }^{s} \boldsymbol{G}_{P}=m_{P}{ }^{S} \boldsymbol{v}_{P},{ }^{s} \boldsymbol{G}_{L}=m_{L}{ }^{S} \boldsymbol{v}_{L} .
$$

where $m_{P}$ and $m_{L}$ are the platform and the load masses, respectively.

Equation (3.25) shows the angular momentum of the system composed by the moving platform and the embarked load, about the center of the main crosshead, which is a fixed point, using $S$ frame [35].

$$
{ }^{S} \boldsymbol{H}_{O}={ }^{S} \boldsymbol{H}_{P}+{ }_{O}^{S} \widetilde{\boldsymbol{r}}_{P}{ }^{S} \boldsymbol{G}_{P}+{ }^{S} \boldsymbol{H}_{L}+{ }_{O}^{S} \widetilde{\boldsymbol{r}}_{L}^{S} \boldsymbol{G}_{L}
$$

where ${ }_{O}^{S} \widetilde{\boldsymbol{r}}_{P}$ and ${ }_{O}^{S} \widetilde{\boldsymbol{r}}_{L}$ are the tilde matrices of the position vector of the platform and load CM using S frame.

Using Euler's Law, Eq. (3.26) gives the torque ${ }^{s} \boldsymbol{M}_{O}$ acting on the moving platform.

$$
{ }^{S} \boldsymbol{M}_{O}={ }^{S} \dot{\boldsymbol{H}}_{O}+{ }_{F}^{S} \tilde{\boldsymbol{\omega}}_{S}{ }^{S} \boldsymbol{H}_{O}
$$

The torque acting on the moving platform is generated by the force vector of the actuators, by the gravity force and by the universal joint constrains, as shown in Eq. (3.27).

$$
{ }^{S} \boldsymbol{M}_{O}={ }^{S} \boldsymbol{T}^{F}\left(\left(m_{P O}{ }_{O}^{F} \tilde{\boldsymbol{r}}_{P}+m_{L}{ }_{O}^{F} \tilde{\boldsymbol{r}}_{L}\right){ }^{F} \boldsymbol{g}+{ }_{O}^{F} \tilde{\boldsymbol{r}}_{C 31}{ }^{F} \boldsymbol{F}_{a 1}+{ }_{O}^{F} \tilde{\boldsymbol{r}}_{C 32}{ }^{F} \boldsymbol{F}_{a 2}\right)+{ }^{S} \boldsymbol{M}_{J}
$$


where ${ }^{F} \boldsymbol{g}$ is the gravity vector, ${ }_{O}^{F} \widetilde{\boldsymbol{r}}_{C 31}$ and ${ }_{O}^{F} \widetilde{\boldsymbol{r}}_{C 32}$ are the tilde matrices of the position vector of the points $C_{31}$ and $C_{32}$, and ${ }^{S} \underline{M}_{J}$ is the torque due to the universal joint constraint, given by Eq. (3.28).

$$
{ }^{S} \boldsymbol{M}_{J}={ }^{S} \boldsymbol{T}^{R}\left[\begin{array}{c}
0 \\
0 \\
M_{J}
\end{array}\right]
$$

Using Eq. (3.26) and (3.27), the angular accelerations are calculated as

$$
\ddot{\beta}=\frac{\tau_{2}-\tau_{3} \tan \alpha-c_{4} \dot{\alpha} \dot{\beta}}{c_{3}}, \ddot{\alpha}=\frac{\tau_{1}-c_{2} \dot{\beta}^{2}}{c_{1}}
$$

where the vector components $\tau_{1}, \tau_{2}$ and $\tau_{3}$ are introduced:

$$
\begin{aligned}
& {\left[\begin{array}{l}
\tau_{1} \\
\tau_{2} \\
\tau_{3}
\end{array}\right]={ }^{S} \boldsymbol{T}^{F}\left(\left(m_{P O}{ }_{O}^{F} \tilde{\boldsymbol{r}}_{P}+m_{L}{ }_{O}^{F} \tilde{\boldsymbol{r}}_{L}\right)^{F} \boldsymbol{g}+{ }_{O}^{F} \tilde{\boldsymbol{r}}_{C 31}{ }^{F} \boldsymbol{F}_{a 1}+{ }_{O}^{F} \tilde{\boldsymbol{r}}_{C 32}{ }^{F} \boldsymbol{F}_{a 2}\right),} \\
& c_{1}=I_{1 P}+m_{p} l_{p}{ }^{2}+I_{1 L}+m_{L} l_{L}^{2}, \\
& c_{2}=\left(c_{1}-I_{3 P}-I_{3 L}\right) \sin \alpha \cos \alpha, \\
& c_{3}=c_{1} \cos \alpha+\left(I_{3 P}+I_{3 L}\right) \tan \alpha \sin \alpha . \\
& c_{4}=-\left(2 c_{1}-I_{3 P}-I_{3 L}\right) \sin \alpha+\left(I_{3 P}+I_{3 L}\right) \tan \alpha \cos \alpha .
\end{aligned}
$$

Neglecting the actuators inertia, the forces of the HA1 and HA2 are given by

$$
{ }^{F} \boldsymbol{F}_{a 1}=\frac{{ }_{O}^{F} \boldsymbol{r}_{C 31}}{\left|{ }_{O}^{F} \boldsymbol{r}_{C 31}\right|} F_{a 1},{ }^{F} \boldsymbol{F}_{a 2}=\frac{{ }_{O}^{F} \boldsymbol{r}_{C 32}}{\left|{ }_{O}^{F} \boldsymbol{r}_{C 32}\right|} F_{a 2},
$$

where $\frac{{ }_{O}^{F} \boldsymbol{r}_{C 31}}{\left|{ }_{O}^{F} \boldsymbol{r}_{C 31}\right|}$ and $\frac{{ }_{O}^{F} \boldsymbol{r}_{C 32}}{\left|{ }_{O}^{F} \boldsymbol{r}_{C 32}\right|}$ are unit vectors in the HA1 and HA2 directions, respectively. $F_{a 1}$ and $F_{a 2}$ are the forces magnitudes of the HA1 and HA2, respectively, and are calculated in Chapter 4.

\section{2.}

Homokinetic platform

The homokinetic platform gives an alternative for the conventional TVC design used in the gimbaled platform. In this section, the peculiarities of the 
homokinetic platform are highlighted. To avoid the repetition of several parts of the last section, the following kinematic and dynamic model focus on the differences. It is recommended to read the previous section before this section for a better comprehension.

\subsection{1.}

\section{Kinematics}

As an alternative for the gimbaled platform, this Thesis proposes the replacement of the universal joint that connects the moving table to the fixed base by a CVJ, thus forming a homokinetic platform. Figure 3.4 shows the homokinetic platform with the constant velocity joint and two IMUs.

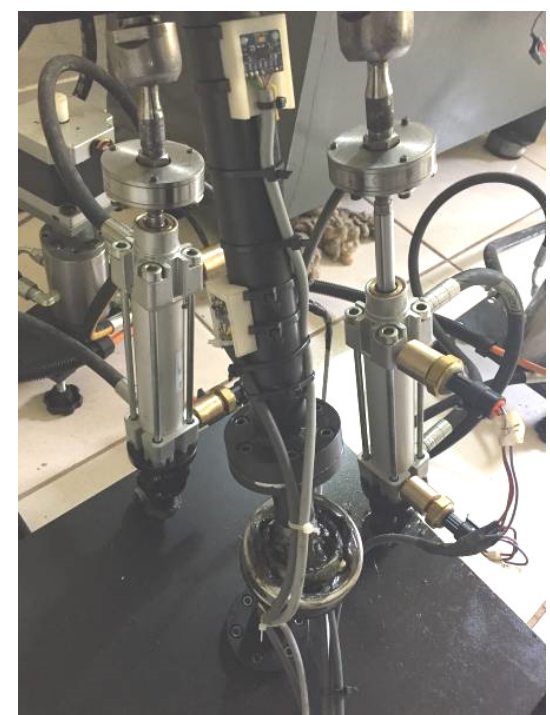

Figure 3.4: Homokinetic platform photo.

Figure 3.5 depict a typical automotive CVJ. 


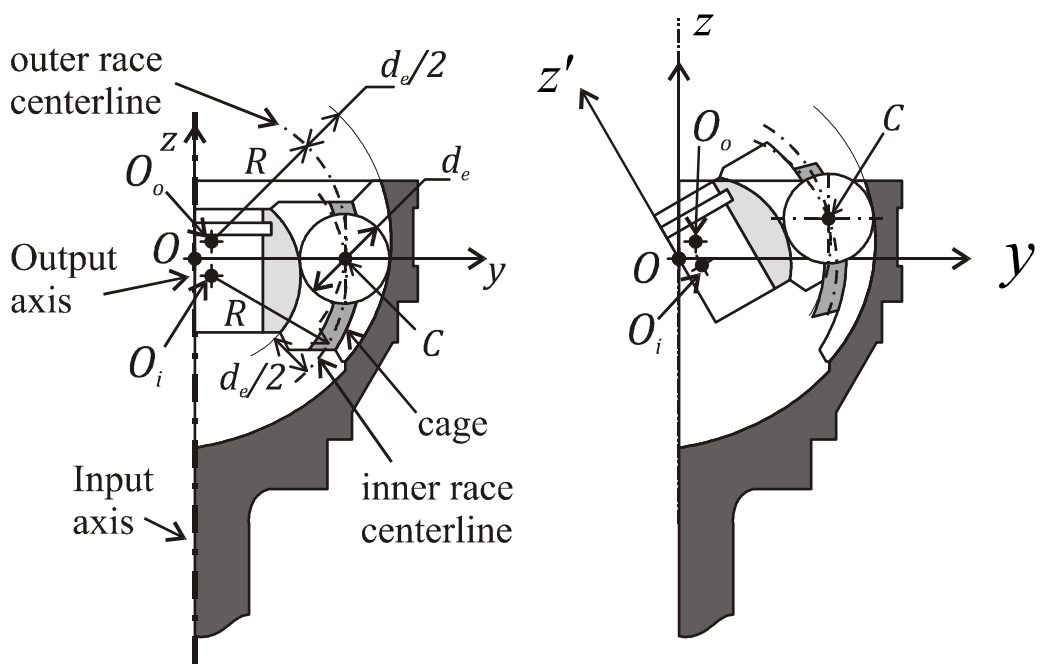

Figure 3.5: CVJ with aligned axes (left) and not aligned (right).

Figure 3.5 presents four rigid bodies:

- the sphere with diameter $d_{e}$;

- the input axis with the outer racetrack centered in $O_{o}$ where the sphere travels;

- the output axis with the inner racetrack centered in $O_{i}$ where the sphere also travels;

- and the cage that held the sphere in a plane that passes through the point $O$.

In fact, an automotive CVJ has six or eight spheres (omitted in Fig. 3.5 due to the axisymmetric configuration). The outer racetrack in centered in $O_{o}$ and the inner racetrack is centered in $O_{i}$. The both racetrack centerline have radius $R$. In Fig. 3.5, the inner racetrack centerline belongs to the yz-plane. $C$ is the point where the inner and the outer racetrack intercepts and it is also the sphere center.

The point $O$ is a fixed point where the centerlines of the axes intersect, as showed by Fig. 3.5 (right). Thus, with this constraint of a fixed point, to know the position of the output axis, it is required to know just its angular orientation, like a ball joint. Indeed, the joint gives an angular restriction between the axes; hence the orientation of the output axis can be given by two angular variables.

In this work, a simple rotation matrix expresses the attitude of the CVJ output axis as a function of two angular variables. Figure 3.6 depicts the proposed method to study the CVJ kinematics. 


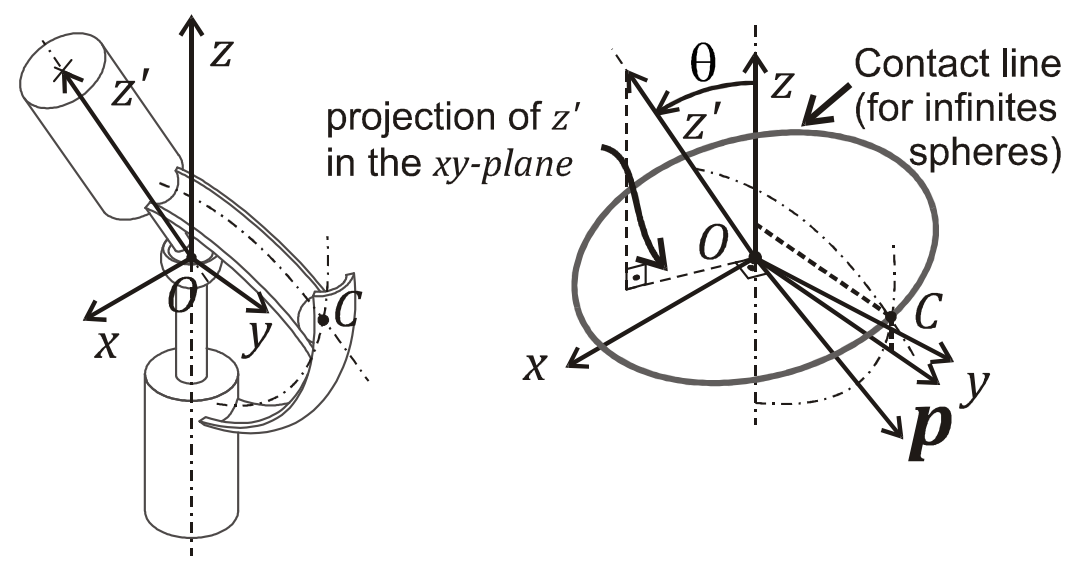

Figure 3.6: CVJ kinematics.

Figure 3.6 (left) shows the inner race and the outer race of a sphere in a schematic CVJ. The lower axis is considered as the input axis and the upper axis is the output axis. The input axis is fixed. The $z^{\prime}$-axis is attached to the output axis. The $x$-axis and the $y$-axis are attached to the input axis, hence they are fixed. The cage is not shown in this figure, but it is responsible for keeping the sphere center in the races centerlines contact point.

Figure 3.6 (left) presents only one sphere of a CVJ; however, CVJs usually have six or eight spheres. The amount of sphere can be increased, but it is limited by the manufacturing process. There are no kinematic restrictions to the amount of spheres. Hence, in Fig. 3.6 (right) is presented a CVJ with an infinite amount of spheres. The $C$-point of the case with only one sphere is still shown, but in this case altogether the sphere centers becomes a line. In this case, all the inners and outers races interception form a contact line, that also contains the sphere centers. Moving the output axis, for any instantaneous movement, there are two sphere centers that do not move. The center of these two spheres forms a line that also passes through the fixed point $O$ and belongs to the $x y$-plane. Hence, an invariant vector $\boldsymbol{p}$, belonging to this line, is introduced in the figure. The invariant vector $\boldsymbol{p}$ is the Euler vector known from the previous chapter, and the angle of rotation of the output axis around $\boldsymbol{p}$ is $\theta$. The direction of $\boldsymbol{p}$ is chosen as to result in a positive rotation angle $\theta$.

This approach is used in Fig. 3.7 that depicts the kinematics of the homokinetic platform. 


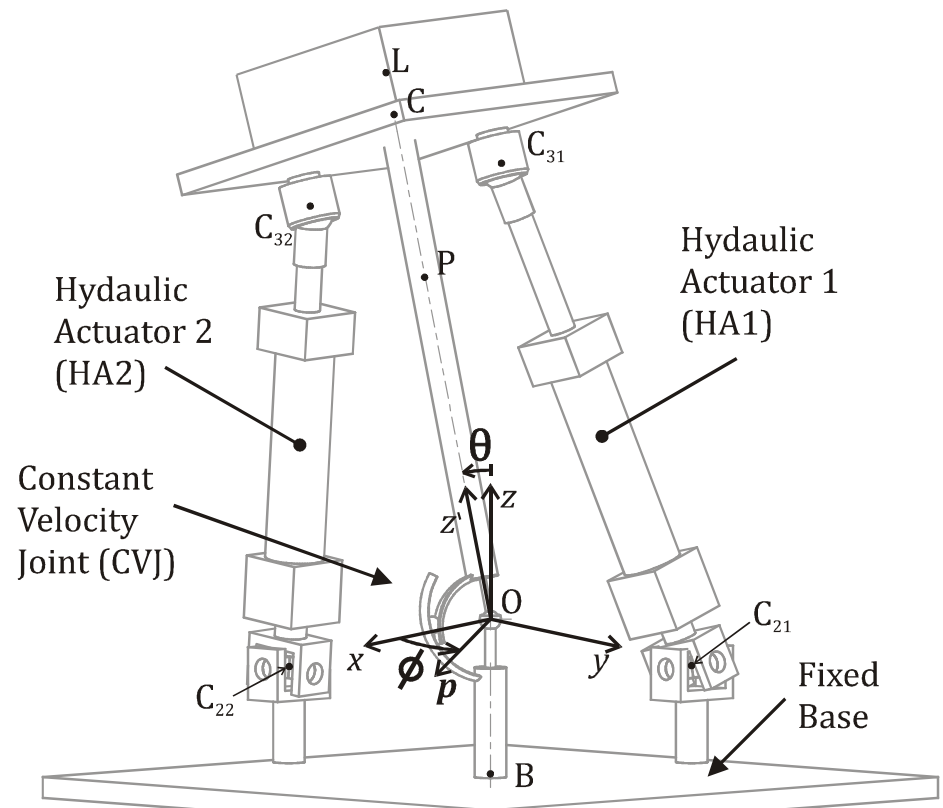

Figure 3.7: Homokinetic platform kinematic scheme.

The kinematics of this homokinetic platform is similar to the gimbaled platform. Although, the kinematic study is performed using only two references frames: $F$ fixed to the base and $S$ attached to the moving platform. And the rotation between the frames has the following indication,

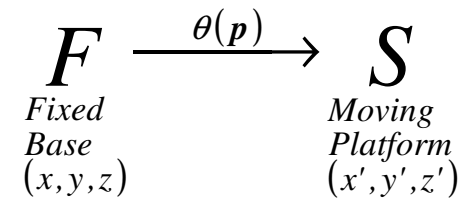

The angle $\theta$ is the angle that tilts the platform, i.e., the angle between $O C$ and the vertical. The position of $\boldsymbol{p}$ changes in the fixed $(x, y)$ plane and can be written using the angle $\phi$,

$$
\boldsymbol{p}=\left[\begin{array}{lll}
\cos \phi & \sin \phi & 0
\end{array}\right]^{T},
$$

where $\phi$ is the angle between vector $p$ and the $x$-axis.

The attitude matrix ${ }^{F} \boldsymbol{T}^{S}$ that leads from $S$ to $F$ is written in terms of this Euler vector and the rotation angle through the Eq. (2.18), rewritten bellow,

$$
{ }^{F} \boldsymbol{T}^{S}=\boldsymbol{E}+\tilde{\boldsymbol{p}} \sin \theta+(1-\cos \theta) \tilde{\boldsymbol{p}}^{2} .
$$

Hence, the attitude matrix ${ }^{F} \boldsymbol{T}^{S}$ in terms of the rotation angles is, 


$$
{ }^{F} \boldsymbol{T}^{S}=\left[\begin{array}{ccc}
1-(1-\cos \theta) \sin ^{2} \phi & (1-\cos \theta) \sin \phi \cos \phi & \sin \theta \sin \phi \\
(1-\cos \theta) \sin \phi \cos \phi & 1-(1-\cos \theta) \cos ^{2} \phi & -\sin \theta \cos \phi \\
-\sin \theta \sin \phi & \sin \theta \cos \phi & \cos \theta
\end{array}\right] .
$$

The angular velocity of a body is a result of the variation in time of the attitude matrix and can be defined as the antisymmetric matrix of Eq. (2.19), Omitting the unnecessary indexes, the angular velocity of the moving platform using the $S$ frame is given by,

$$
{ }_{F}^{S} \boldsymbol{\omega}_{S}=\dot{\boldsymbol{\theta}}+\sin \theta \dot{\boldsymbol{p}}-(1-\cos \theta) \tilde{\boldsymbol{p}} \dot{\boldsymbol{p}},
$$

Hence,

$$
{ }_{F}^{S} \omega_{S}=\left[\begin{array}{c}
\cos \phi \dot{\theta}-\sin \theta \sin \phi \dot{\phi} \\
\sin \phi \dot{\theta}+\sin \theta \cos \phi \dot{\phi} \\
(\cos \theta-1) \dot{\phi}
\end{array}\right] .
$$

The rest of the necessary calculations for the homokinetic platform is identical to the previously made for the gimbaled configuration. And can be obtained using the attitude matrix of Eq. (3.39) and the angular velocity of Eq. (3.41) in Eqs. (3.8)-(3.18).

\subsection{2. Dynamics}

The torque acting on the homokinetic moving platform is similar to the gimbaled configuration. The difference is the torque due to the homokinetic constraint, given by Eq. (3.42),

$$
{ }^{s} \boldsymbol{M}_{J}=\left[\begin{array}{c}
0 \\
0 \\
M_{J}
\end{array}\right] \text {. }
$$

Considering this CVJ constraint in Eq. (3.27) and recalculating the torque acting on the moving platform of the Eq. (3.26) with the homokinetic attitude matrix and angular velocity, the angular accelerations are calculated as

$$
\ddot{\theta}=\frac{\tau_{1} \cos \phi+\tau_{2} \sin \phi+c_{3} \sin \theta \dot{\phi}^{2}}{c_{1}}, \ddot{\phi}=\frac{\tau_{2} \cos \phi-\tau_{1} \sin \phi-c_{2} \dot{\phi} \dot{\theta}}{c_{1} \sin \theta}
$$

where: 


$$
\begin{aligned}
& {\left[\begin{array}{l}
\tau_{1} \\
\tau_{2} \\
\tau_{3}
\end{array}\right]={ }^{S} \boldsymbol{T}^{F}\left(\left(m_{P}{ }_{O}^{F} \tilde{\boldsymbol{r}}_{P}+m_{L}{ }_{O}^{F} \widetilde{\boldsymbol{r}}_{L}\right){ }^{F} \boldsymbol{g}+{ }_{O}^{F} \widetilde{\boldsymbol{r}}_{C 31}{ }^{F} \boldsymbol{F}_{a 1}+{ }_{O}^{F} \widetilde{\boldsymbol{r}}_{C 32}{ }^{F} \boldsymbol{F}_{a 2}\right),} \\
& c_{1}=I_{1 P}+m_{p} l_{p}^{2}+I_{1 L}+m_{L} l_{L}^{2}, \\
& c_{2}=2 c_{1} \cos \theta+(1-\cos \theta)\left(I_{3 P}+I_{3 L}\right), \\
& c_{3}=c_{1} \cos \theta+(1-\cos \theta)\left(I_{3 P}+I_{3 L}\right) .
\end{aligned}
$$

The kinematic and dynamic considerations made in this chapter enable the analytical solution of the dynamic behavior of the moving platform. However, it is still needed to compute the input force performed by the actuators. The dynamics of this hydraulic system is analyzed in the next chapter. 


\section{4 \\ Hydraulic control system}

In this Thesis, the TVC system is actuated by two independent hydraulic actuated control systems. Each EHA system has a four-way valve-controlled single-rod linear actuator, hence it is one of the most basic hydraulic controlled system. However, even though it is simple the cost is very high if a servo-valve is necessary. Aiming a low-cost system alternative, a novel control valve is proposed specially developed for this Thesis, called PDHV. This valve is a proportional four-way valve with an underlapped symmetric closed-center rotary spool. And an open-loop stepper motor drives the valve spool through an elastic-coupling. Figure 4.1 shows an exploded view of the PDHV.

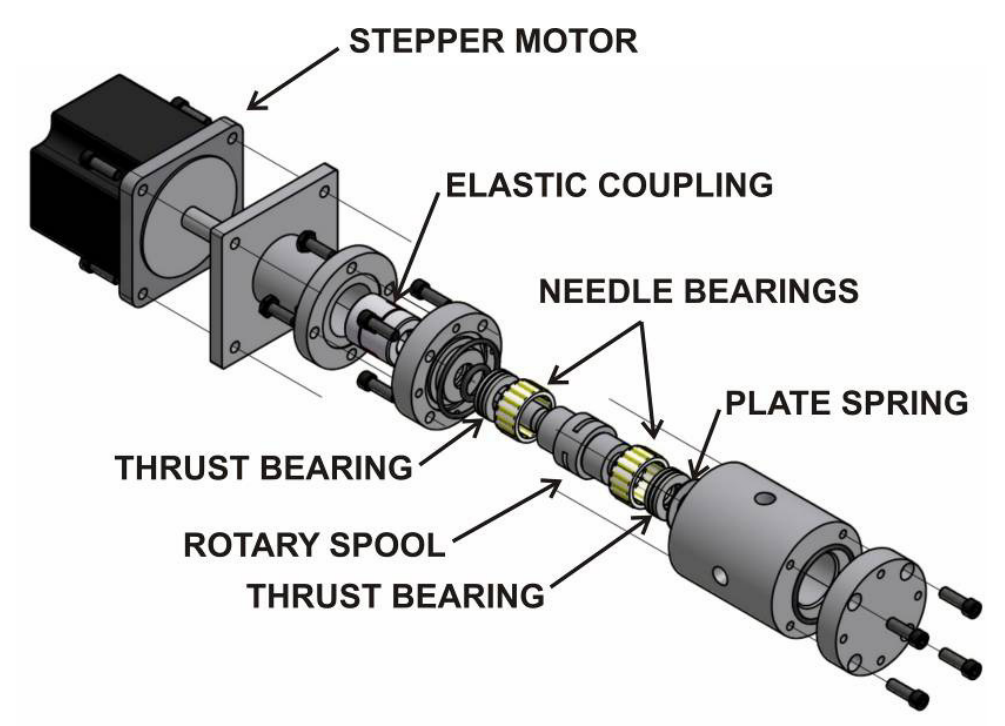

Figure 4.1: PDHV exploded view.

The needle bearings support the rotary spool radial loads due to the asymmetric oil pressure, while the thrust bearings support the axial force generated due to the driven side of the rotary spool which is opened to the atmosphere. A spring plate assures the minimum load on the thrust bearings for their stability. Figure 4.2 depicts one of the PDHVs built. 


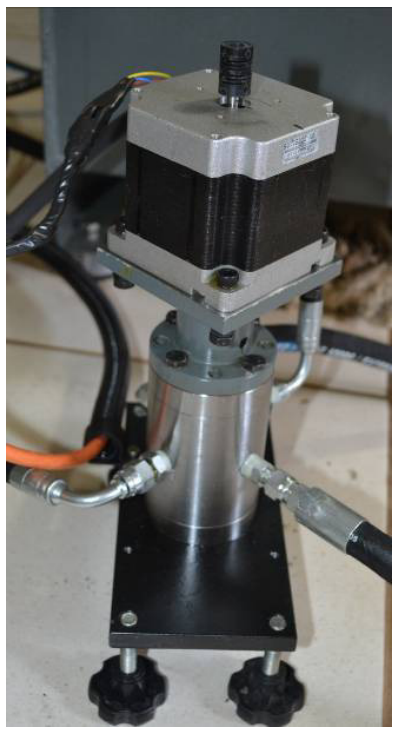

Figure 4.2: PDHV photo.

Each actuator of the platform is controlled by one PDHV, as shown in Fig. 4.3. By changing the valve rotor angle $\theta_{V}$, the pressure drop and the flow rate through the valve change, which changes the pressure in the pipelines and in the actuator chambers, thereby generating a force that moves the piston.

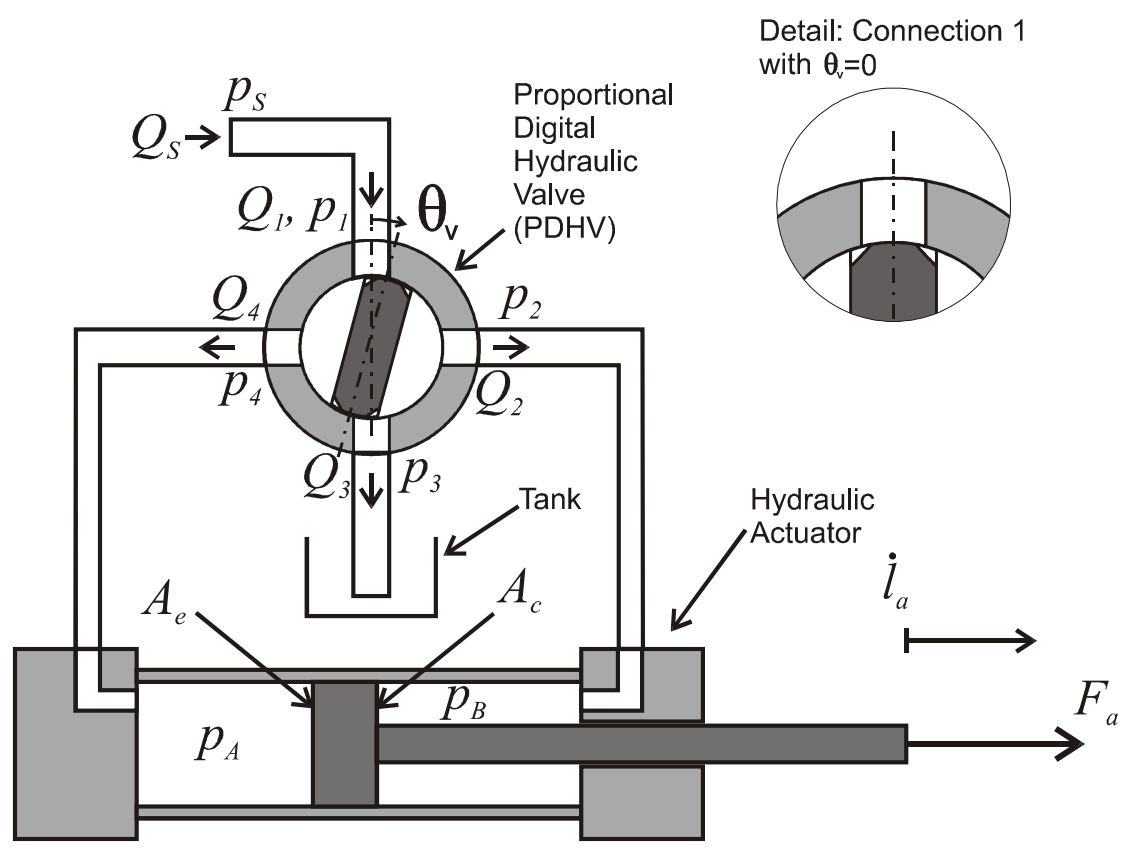

Figure 4.3: Hydraulic scheme. 
In Fig. 4.3, $p_{S}$ and $Q_{S}$ are the supply pressure and flow rate, respectively. The arrows in the pipeline indicate the positive flow sense. $p_{1}, p_{2}, p_{3}$ and $p_{4}$ are the pressures in the valve connections. $Q_{1}, Q_{2}, Q_{3}$ and $Q_{4}$ are the flow rates with the positive direction indicated by the arrows. $p_{A}$ and $p_{B}$ are the pressures in chambers $A$ and $B$, respectively; $A_{e}$ and $A_{c}$ are the areas of the embolus and the crow, respectively; $F_{a}$ is the force acting on the rod; and $i_{a}$ is the piston speed. For a simpler kinematic analysis of the platform, $l_{a}$ is the distance between the centers of the mounting joints of the actuators. The rotary spool has $32 \mathrm{~mm}$ in diameter and each connection hole has $9.5 \mathrm{~mm}$ in diameter.

Using the kinematic model and the measured angular platform position, the actual actuators position $l_{a}$ is calculated. The controller compares the actual positions with the desired ones and changes the valve rotor angle $\theta_{V}$ proportionally to the error.

\section{1. Actuator model}

The platform inertia is much bigger then the actuators inertia. Hence it is neglected and actuator force is given by $[10,36]$,

$$
F_{a}=p_{A} A_{e}-p_{B} A_{c}-F_{f} .
$$

where $F_{f}$ is the friction force.

In the analyzed conditions of the platform, the friction force is well represented as a function of the actuator speed using a Stribeck curve, because the slip velocity on the actuator is significant and a relatively small amount of static/kinetic friction appears in the transition [37]. A more precise model, for example, the LuGre model, shows the time dependence of the friction and could be used in a more general condition or for real-time friction compensation [38].

In the platform simulations, the friction force is obtained experimentally considering constant actuator speed without a load; the fitted function used (continuous line) is depicted in Fig. 4.4 with the experimental data (vertical bars). 


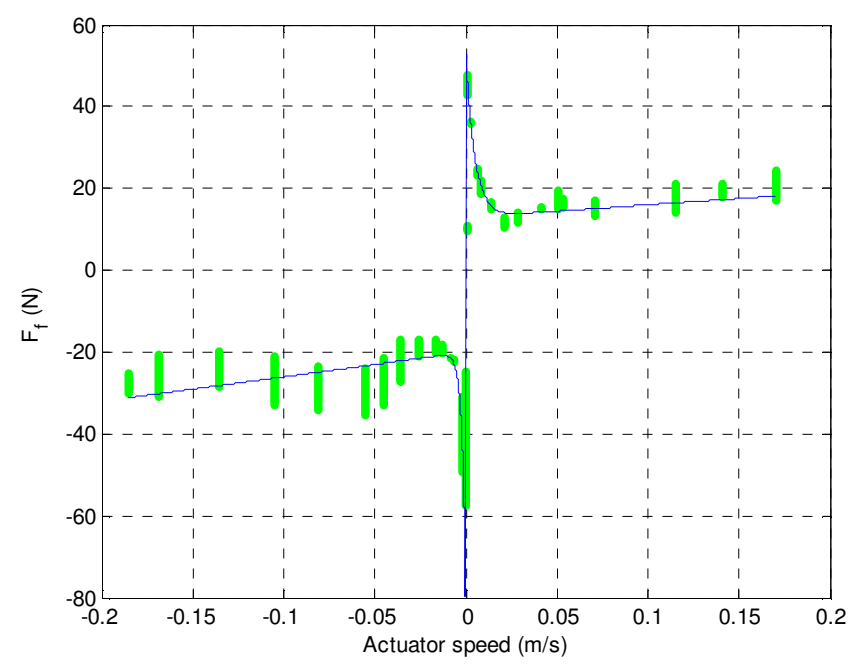

Figure 4.4: Actuator friction force.

The vertical bars have this shape because the experiment is performed for a discrete number of actuator speeds and the variation is due to noise and inaccuracy of the used model. During this experiment, the valve model parameters are also identified.

\section{2. Valve model}

In this work, the pipeline pressure loss due to friction is negligible and is not considered. However, the pump is considered to be capable of maintaining a constant supply pressure during the experiments. This hypothesis was validated.

The pressure drop trough a PDHV is calculated using a variable-area orifice model [10],

$$
Q_{i j}=\alpha_{D i j} A_{i j} \sqrt{\frac{2}{\rho}\left|p_{i}-p_{j}\right|} \operatorname{sign}\left(p_{i}-p_{j}\right),
$$

where $Q_{i j}$ is the flow rate between connections $i$ and $j$; these indices vary from 1 to 4 according to the valve connection depicted in Fig. 4.3. $\alpha_{D i j}$ is the discharge coefficient. $A_{i j}$ is the orifice area, which is a function of the valve spool angle $\theta_{V}$, as shown in Fig. 4.5. 


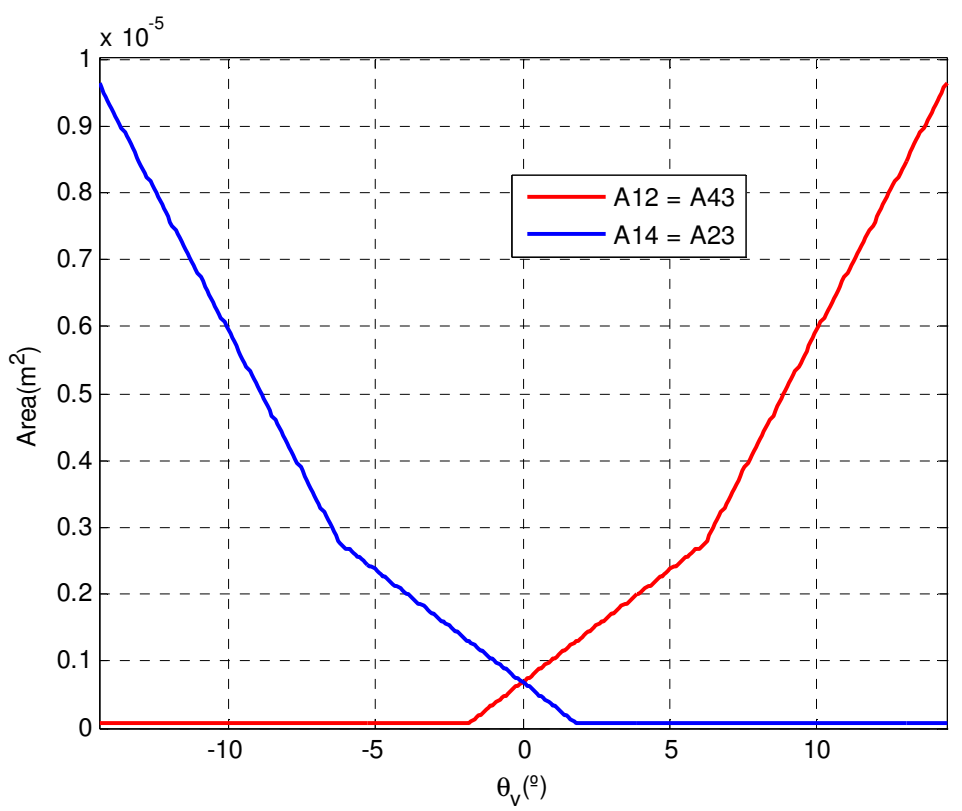

Figure 4.5: Orifice area as a function of the valve position.

Due to the symmetry of the valve, the orifice area between the connections 1 and 2 is numerically equal to the area between connections 4 and 3 . And the orifice area between the connections 1 and 4 is numerically equal to the area between connections 2 and 3 . The function of the orifice area is never zero. It is done to consider the leakage inside the valve and it helps the stability of the numerical simulations.

The continuity equation applied on the hydraulic scheme, considering an incompressible flow, gives the oil flow in the actuators chambers:

$$
Q_{a}=Q_{14}-Q_{43}, Q_{b}=Q_{12}-Q_{23} .
$$

Equations (4.5) and (4.6) show the relationships between the flow rate in the chambers and the speed of the actuators:

$$
Q_{a}=A_{e} i_{a}, Q_{b}=A_{c} i_{a} .
$$

During the experiment that identifies the friction force on the actuator, a discrete number of valve opening positions are demanded. These data are also used to identify the valve parameters. Figure 4.6 shows the valve opening over time for this experiment. 


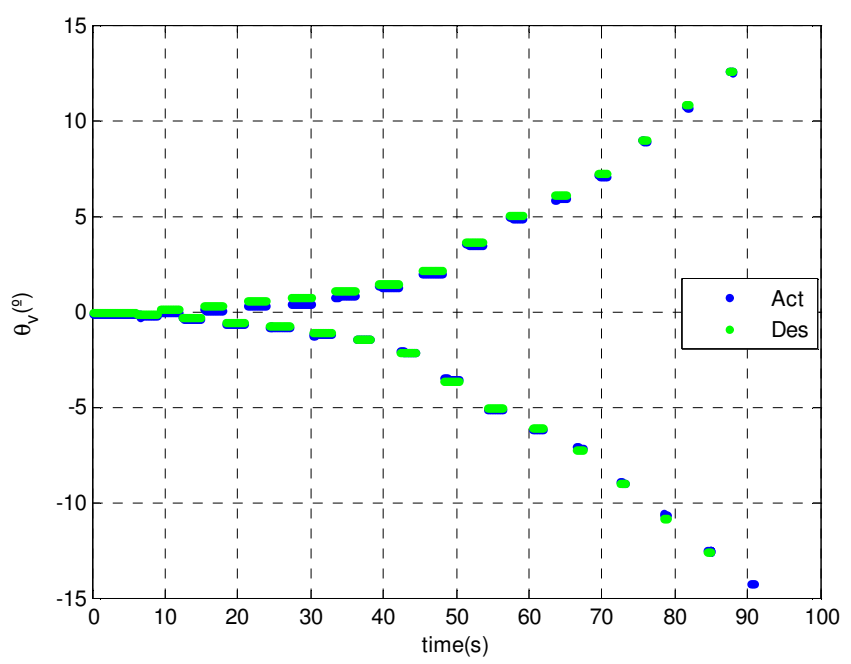

Figure 4.6: Valve opening in no-load experiment.

In this experiment, the discharge coefficient from Eq. (4.2) is also estimated using Eqs. (4.3-4.6). Figure 4.7 shows the oil flow in the actuator chamber $A$ calculated using the speed measure in Eq. (4.5) (green line) and the fitted value for a set of discharge coefficients (blue line).

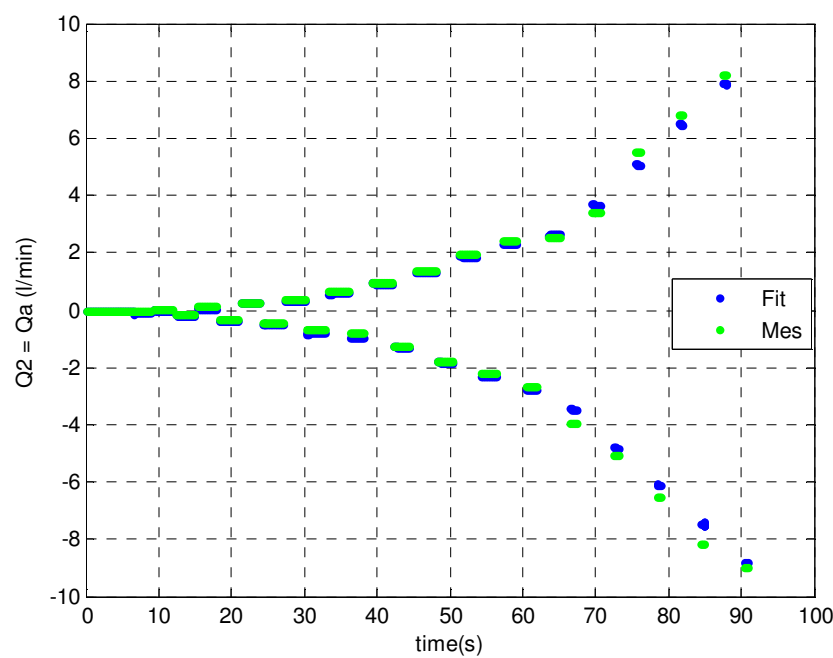

Figure 4.7: Chamber $A$ flow in no-load experiment.

Figure 4.8 shows the oil flow in the actuator chamber $B$ calculated using the speed measure in Eq. (4.6) (green line) and the fitted value the set of discharge coefficients (blue line). 


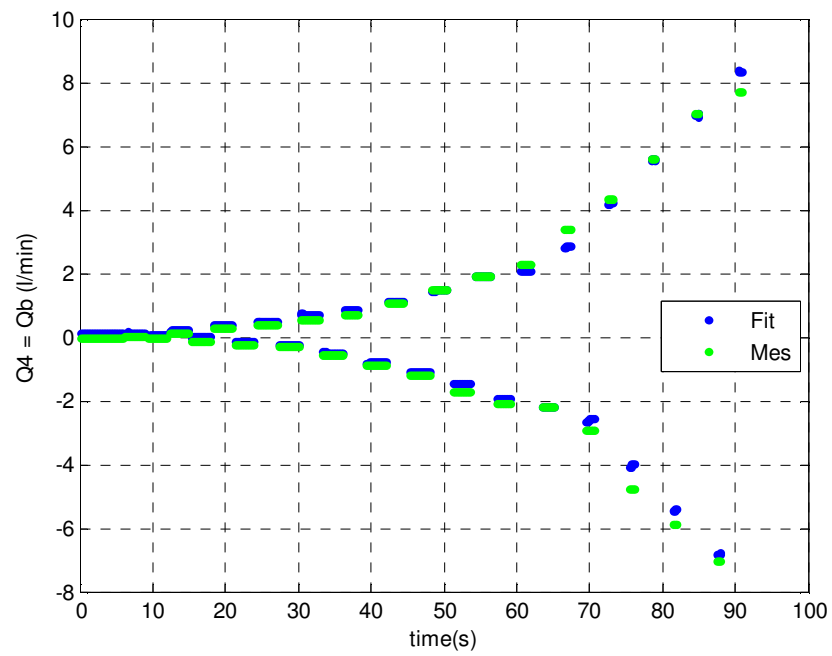

Figure 4.8: Chamber $B$ flow in no-load experiment.

Hence, the chosen set of discharge coefficients and Eq. (4.2) can be used for oil flow modeling in the hydraulic system. The experimental results presented in this section are for HA1, and they are very similar for HA2. Because of the valve axissimetry, the all discharge coefficients used in the fitted flow function are given by,

$\alpha_{D i j}=0.35$.

Using Eqs. (4.2)-(4.7), Fig. 4.9 gives the flow rate characteristic versus the rotary spool shift for several pressure drops. 


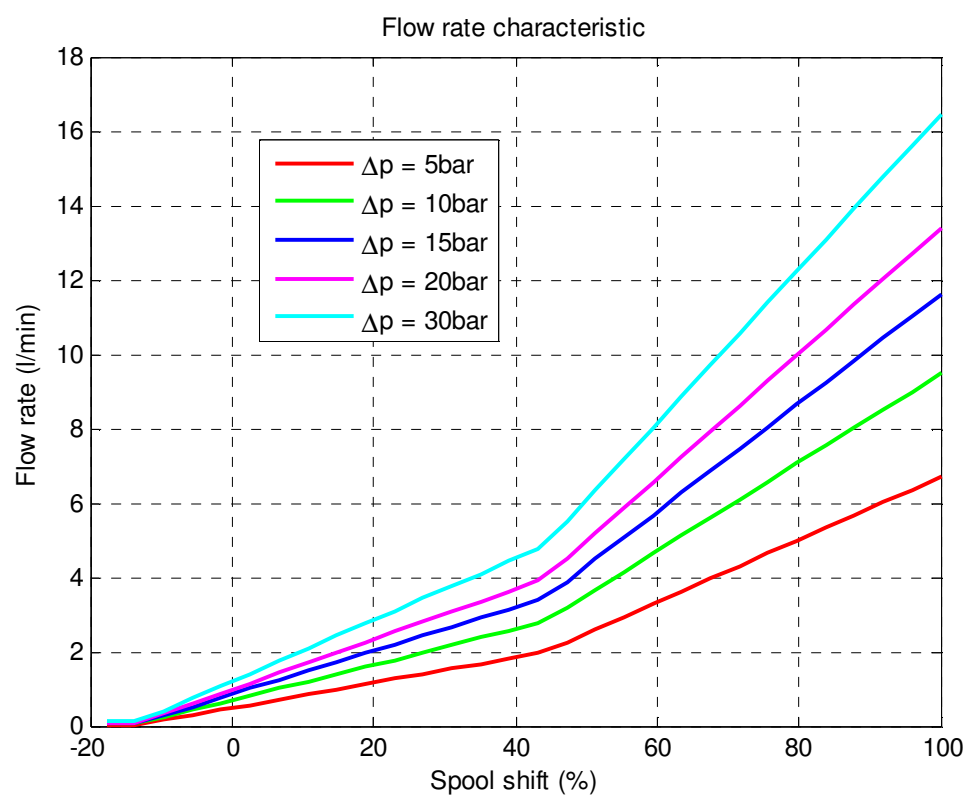

Figure 4.9: Flow rate vs. spool shift.

The non-zero flow for a null spool shift is characteristic of an underlapped valve. In addition, the single rod actuator used in this Thesis gives a positive velocity in a null spool shift condition. The numerical and experimental trials identify that when the spool is deflected form the central position of $-0.08^{\circ}$, the actuators have null velocity in the absence of external forces. Hence, the null valve position $\left(\theta_{V}=0\right)$ is deflected $-0.08^{\circ}$ from the actual central position. This procedure helps the controllability of the single rod cylinder.

The valve is driven by an open-loop stepper motor, where the loss of steps is avoided limiting the maximum angular velocity and acceleration of the spool. The maximum angular velocity and acceleration are obtained with experimental trials where the loss of steps is monitored with a rotary encoder. In addition, during this trial a minimum time of $6.5 \mathrm{~ms}$ (called inversion time) is considered to change the stepper direction. The tests were carried out with 15 bar of supply pressure and with an actuator with no load, where the stepper velocity and acceleration were increased until loss of steps. However, for safety purpose, the valve is driven with half of the maximum angular velocity and acceleration obtained in this trial. Thereby, the maximum valve angular velocity is restricted to $21.0 \mathrm{rad} / \mathrm{s}$ and the acceleration to 1,613 rad/s $\mathrm{s}^{2}$, which gives valve work envelope shown in Fig. 4.10. 


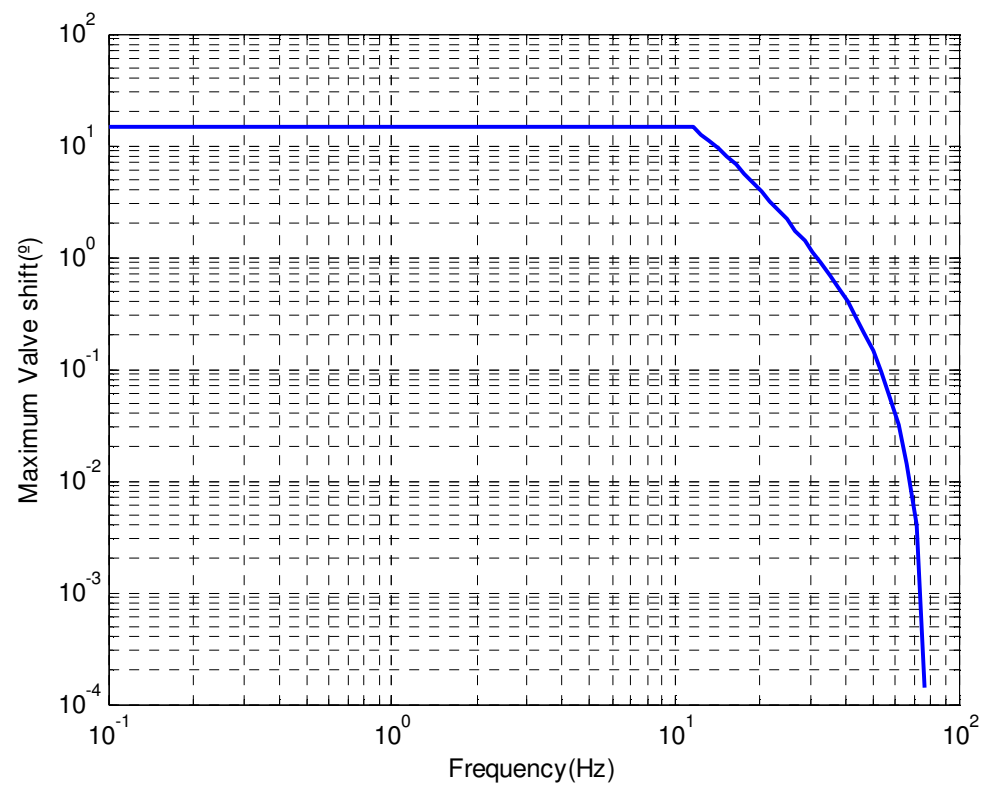

Figure 4.10: Valve shift limitation caused by the stepper controller

In the working envelope, until $11.5 \mathrm{~Hz}$, the valve shift is limited by its maximum stroke of $14.4^{\circ}$. Between $11.5 \mathrm{~Hz}$ and $15.4 \mathrm{~Hz}$, the limitation is caused by the inversion time, maximum angular velocity and acceleration of the openloop stepper control. Between $15.4 \mathrm{~Hz}$ and $76.9 \mathrm{~Hz}$, the limitation is caused by the inversion time and the maximum angular acceleration. After $76.9 \mathrm{~Hz}$, the valve spool does not move, because the minimum inversion time is not reached.

\section{3.}

\section{Other model considerations}

The electrohydraulic power unit has two sets of vane pumps that collect oil through filter elements from a reservoir. Each pump is driven by an AC motor. They also have relief valves to limit the maximum supply pressure. Ensuring that the supply pressure is constant during the system operation, all the modeling of the power unit can be neglected. During the above no load experiment, this hypothesis is tested looking at the supply pressure. Figure 3.9 shows the chambers and supply pressures throughout the no load experiment. 


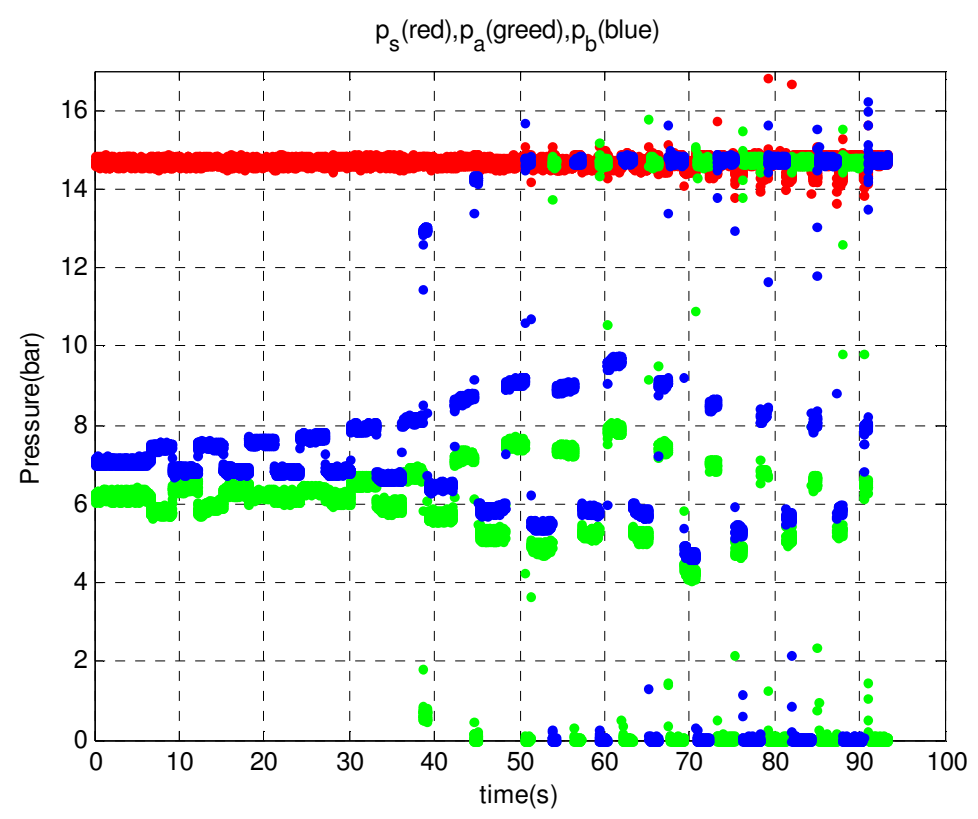

Figure 4.11: Supply and chambers pressure during no-load experiment.

The supply pressure in Fig. 4.9 falls 0.5 bar when using the maximum actuator speed. Since this maximum reduction is only $3.3 \%$ of the supply pressure, it is considered constant for the numerical simulations.

Another consideration involves the pressure drop in the pipelines. Computing the friction using the Moody Chart [39], the numerical simulation of the platform did not converge. The hypothesis of relative small fluid friction solves this problem. This is validated calculating the pressure loss in each section of the pipeline through the Moody Chart. As result, the maximum loss is $0.2 \mathrm{bar}$ in the supply pipeline, $0.3 \mathrm{bar}$ in the hoses and $0.1 \mathrm{bar}$ in the return to the reservoir. The pressure drop is proportional to the actuator speed, thus for low speed it tends to zero. The supply pressure is about 15 bar, hence it is concluded that the pressures losses are relatively small throughout the entire operation range of EHA system.

Furthermore, the dynamics of the hydraulic hoses is not considered. And it is considered that the fluid temperature variation between the tests does not affect the parameters identified and the performance of the system. This hypothesis is validated repeating the no load experiment over a temperature variation between $25^{\circ} \mathrm{C}$ and $35^{\circ} \mathrm{C}$. In this range, the identified parameters and the performance of the 
system does not vary significantly. Thereby, the allowed temperature during the tests is in this range.

In this chapter, the model of the hydraulic system was presented. Gathering with the dynamics model presented in the previous chapter, only the controller model is needed to perform numerical simulations. The controller model is object of the next chapter with the description of the DAQ and the attitude estimation system. 


\section{5 \\ Data acquisition and control system}

Motivated by building a more accessible system, in this Thesis there is a constant quest to reduce costs. The proposal of a novel low-cost control valve does not make sense if the cost of the peripheral system is increased. Thus, in this chapter it is depicted the DAQ system specially built for this work.

Figure 5.1 shows the entire electrical system.

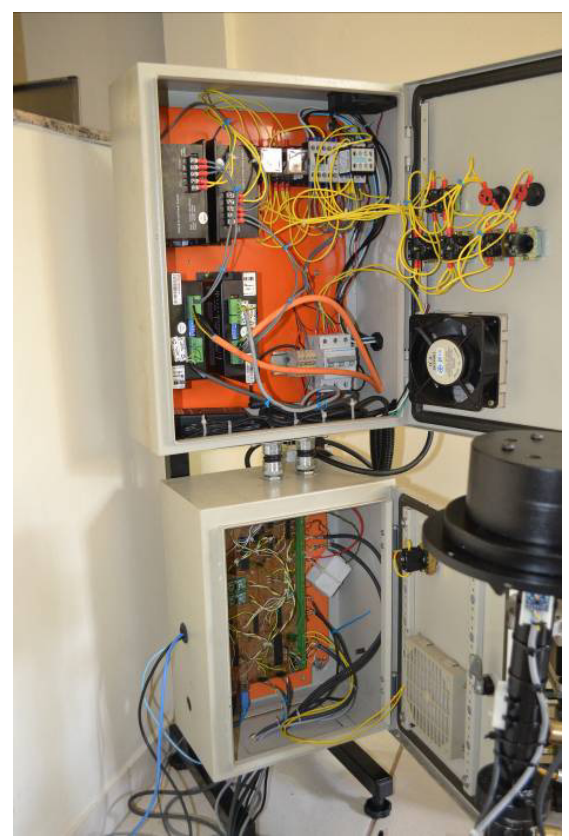

Figure 5.1: Electrical peripheral system.

In this figure, the upper box drives the pumps AC motors and the steppers motors. The bottom box houses de DAQ system.

\section{1. \\ DAQ system}

The data acquisition system (DAQ) consists of two customized microcontrolled circuits. One receives the sensors data and sends these data to a computer with a MATLAB running script. The other one receives the desired 
valves positions from MATLAB script and sends it to the valves controllers. The valves controllers are a variable frequency $(100 \mathrm{~Hz}$ to $25 \mathrm{kHz})$ open-loop microcontrolled circuit specially built for the PDHV. Figure 5.2 shows the DAQ operation scheme.

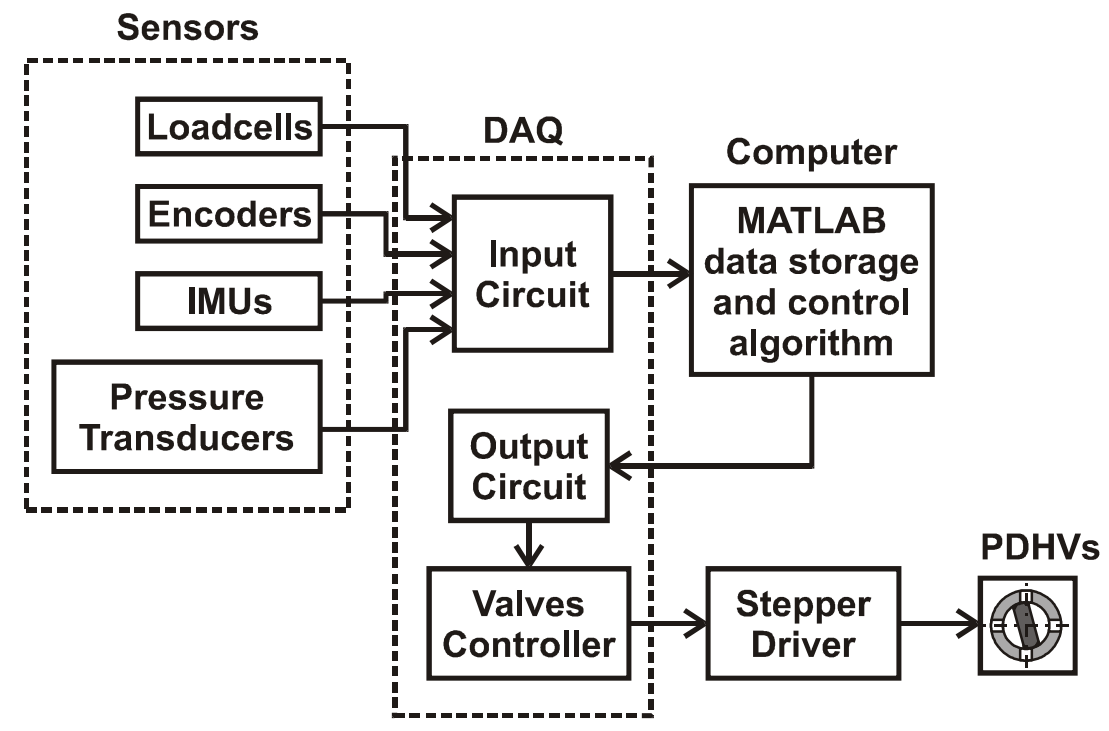

Figure 5.2: DAQ system.

The input circuit has an acquisition frequency of $50 \mathrm{~Hz}$, and it is also the control frequency. The MATLAB script runs the control algorithm. Thereby the time between receiving the sensor data and the response of output variables depends on the complexity of the algorithm and the computer speed. For the proposed algorithm the delay is less than $1 \mathrm{~ms}$, or about $5 \%$ of the control period.

\section{2.}

Controller

The platform has a decentralized proportional controller, hence the position control of each actuator is independent. Figure 5.3 depicts the control loop of the platform. 


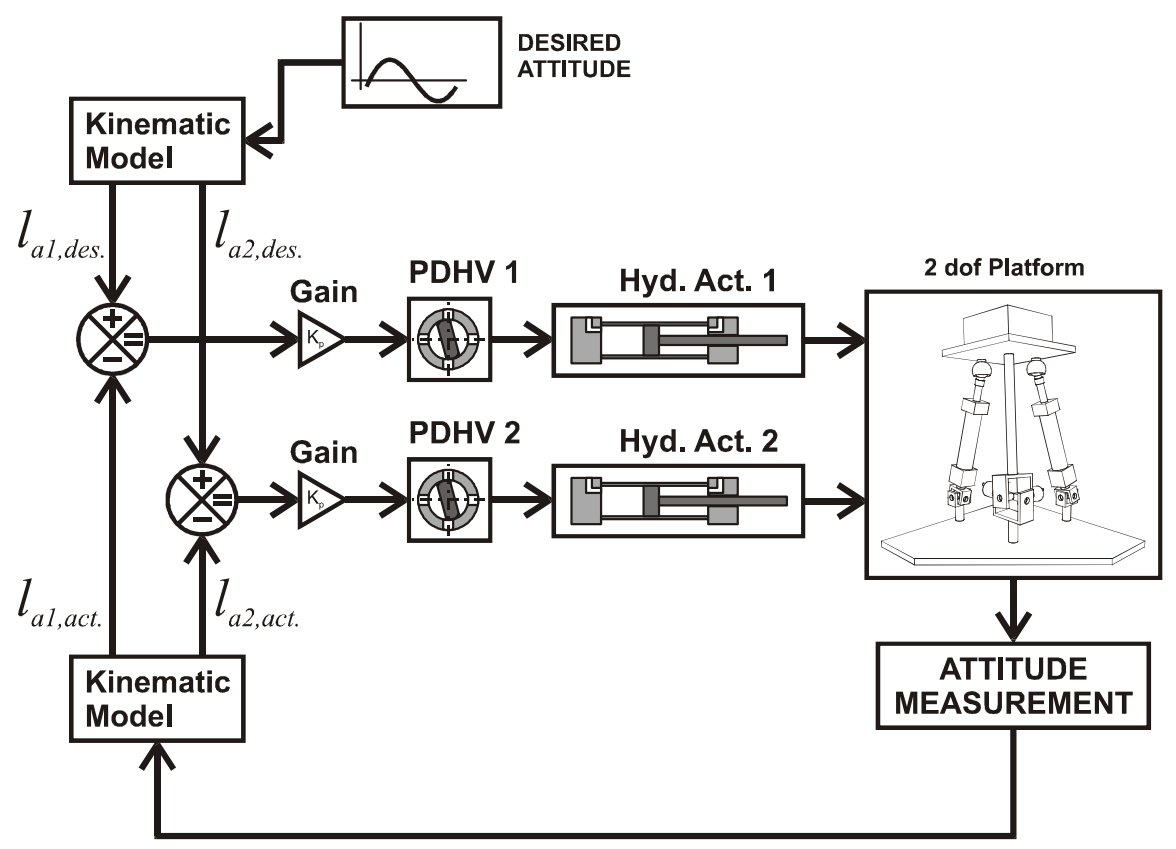

Figure 5.3: 2-DOF platform controller.

The control loop receives a desired angular position for the platform. Using the kinematic model, the desired actuator positions are calculated and compared with the actual positions. The errors are multiplied by a gain and sent to the PDHV controller. The PDHV controller has an open loop to control a stepper motor attached to the valve spool. Accordingly to the spool position, the oil flow to the actuators changes, thus its position changes and the orientation of the platform changes. The proportional gain is chosen trying several values in the simulations, Fig. 5.4 shows the step response of HA1 for several gains on the gimbaled platform. 


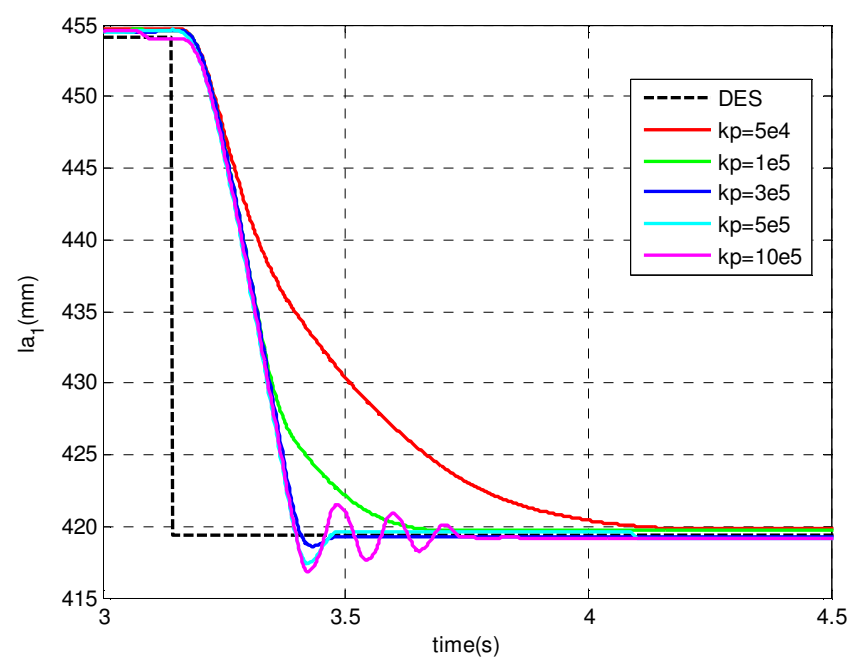

Figure 5.4: Step response of HA1 with different gains.

Using Fig. 5.4, the $\mathrm{k}_{\mathrm{P}}=1 \cdot 10^{5}$ is chosen because there are no oscillations and no overshoots. Due to the symmetry of the actuating system, the HA2 has a similar response, thus the same gain is used. This gain value is considered until Section 6.5, unless another controller is proposed.

In the case of the gimbaled platform, the attitude is measured through angular transducers. Two incremental rotary encoders measure the angular displacements of the main universal joint crosshead. In the homokinetic platform the attitude estimation system, described in the Section 5.4, gives the angular position of the platform.

\section{3.}

Sensors

In this section, the sensors are presented. There is a pressure transducer plugged in a hole on each actuators cap to measure the chambers pressure. The supply pressure is also measured with this sensor. These sensors are built by Sensata Technologies, with the code 100CP2-74, operate between 0 and 250 psi and the resolution is $62.5 \mathrm{psi} /$ Volt.

The measurements of angular velocity and linear acceleration are done by the IMU MPU-6050 from the InvenSense. Although there are several options of range in the IMU, all the experiments of this Thesis have the selected range of \pm 2 $g$ for the accelerometer and $\pm 250 \%$ for the angular velocity. The internal low- 
pass filter is set to $50 \mathrm{~Hz}$. The triaxial gyroscope and the triaxial accelerometer work with 16-bit resolution. The data are read using the $\mathrm{I} 2 \mathrm{C}$ communication protocol.

During the inverted pendulum and no load experiments the position of the PDHV spool is measured with a 1.000 PPR incremental rotary encoder. Manufactured by Autonics with the code E40 S6-1000-3-1-24, it has two phases in quadrature, hence the resolution is $0.09^{\circ}$ /increment

The angular position of the universal joint crosshead of the gimbaled platform is measured with two $500 P P R$ incremental rotary encoders. With two phases in quadrature, the resolution is $0.18 \%$ increment. It is built by Cytron Technologies with the code B-106-23983. This encoder is also used to measure the angular position of the inverted pendulum.

There is a pancake style load cell on each actuator. This load cell was designed specifically for this Thesis. Figure 5.5.a shows an exploded view of the load cell. Figures 5.5.b-c present photos of the assembled load cell. And Figure 5.5.d depicts the strain gage position on the deformable element.

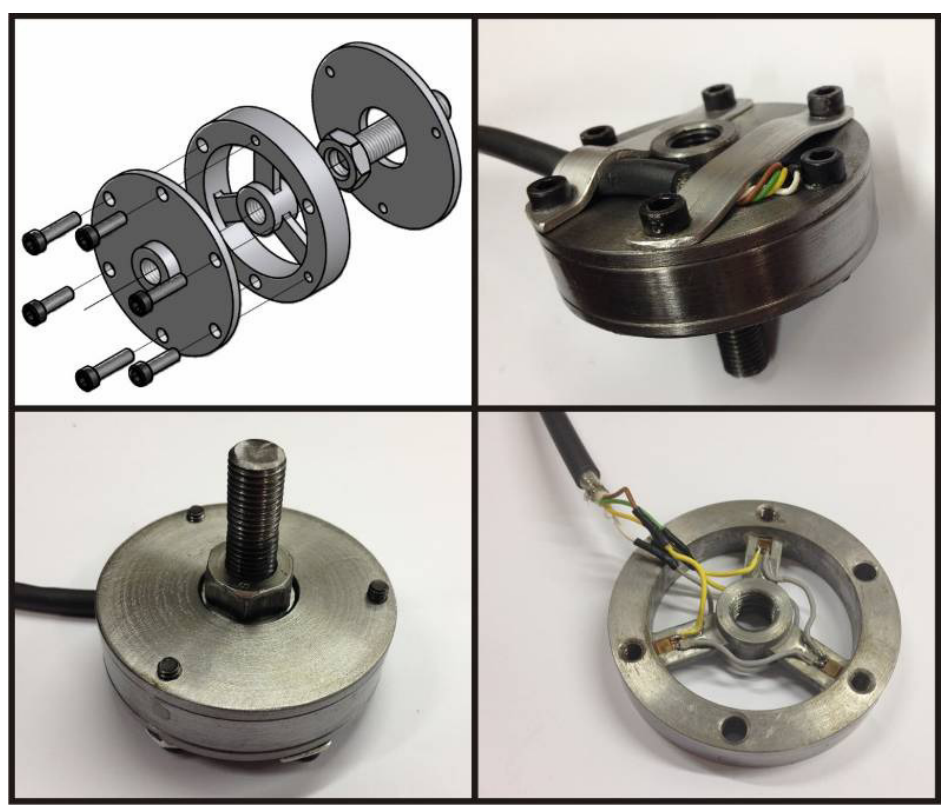

Figure 5.5: Load cell

a (upper left) - Exploded view, b (upper right) - Back view of the assembly, $c$ (bottom left) front view of the assembly, $d$ (bottom right) - the deformable element with strain gages. 
This load cell has six strain gages in half bridge. The deformable element is projected to support $2.500 \mathrm{~N}$ without yielding. It was built in $1045 \mathrm{SAE}$ steel on a tri-axis CNC milling.

\section{4.}

Attitude estimation

To compute the orientation of the platform, a 6-dof IMU is attached to the moving platform. The accelerometer data consist of the linear acceleration of the sensor measured in the embarked frame $\left({ }^{S} \boldsymbol{a}\right)$,

$$
{ }^{s} \boldsymbol{s}={ }^{s} \boldsymbol{a}-{ }^{s} \boldsymbol{g}=\left[\begin{array}{c}
X_{a} \\
Y_{a} \\
Z_{a}
\end{array}\right]
$$

where $X_{a}, Y_{a}$ and $Z_{a}$ are the accelerometer data from the IMU.

Using the kinematic model of the homokinetic platform, the gravity in the embarked frame $S$ is given by,

$$
{ }^{S} \boldsymbol{g}={ }^{S} \boldsymbol{T}^{F F} \boldsymbol{g}=g\left[\begin{array}{c}
-\sin \phi \sin \theta \\
\cos \phi \sin \theta \\
\cos \theta
\end{array}\right] .
$$

Considering ${ }^{S} \boldsymbol{a}=0$ in Eq. (5.1),

$$
\left[\begin{array}{c}
X_{a} \\
Y_{a} \\
Z_{a}
\end{array}\right]=g\left[\begin{array}{c}
-\sin \phi \sin \theta \\
\cos \phi \sin \theta \\
\cos \theta
\end{array}\right] \text {. }
$$

Hence, if $\theta \neq 0, \phi$ from the accelerometer data will be given by

$$
\phi_{a}=\operatorname{atan} 2\left(-X_{a}, Y_{a}\right) \text {. }
$$

For $\theta$, two options are available:

$$
\theta_{a}=\operatorname{atan} 2\left(Y_{a} / \cos \phi, Z_{a}\right), \theta_{a}=\operatorname{atan} 2\left(-X_{a} / \sin \phi, Z_{a}\right) \text {. }
$$

To keep distance from singularities, Eq. (5.5) is used only when $k \pi-\pi / 4 \leq \phi_{a} \leq k \pi+\pi / 4$, where $k$ is an integer, otherwise Eq. (5.6) is used. With $\phi_{a}$ and $\theta_{a}$, the attitude matrix by the accelerometer data is presented as 


$$
{ }^{F} \boldsymbol{T}_{a}^{S}=\left[\begin{array}{ccc}
1-\left(1-\cos \theta_{a}\right) \sin ^{2} \phi_{a} & \left(1-\cos \theta_{a}\right) \sin \phi_{a} \cos \phi_{a} & \sin \phi_{a} \sin \theta_{a} \\
\left(1-\cos \theta_{a}\right) \sin \phi_{a} \cos \phi_{a} & 1-\left(1-\cos \theta_{a}\right) \cos ^{2} \phi_{a} & -\cos \phi_{a} \sin \theta_{a} \\
-\sin \phi_{a} \sin \theta_{a} & \cos \phi_{a} \sin \theta_{a} & \cos \theta_{a}
\end{array}\right]
$$

The attitude matrix is integrated rearranging the Eq. (3.40),

$$
{ }^{F} \dot{\boldsymbol{T}}^{S}={ }^{F} \boldsymbol{T}^{S}{ }_{F}^{S} \tilde{\boldsymbol{\omega}}_{S} .
$$

The IMU gyroscope gives ${ }_{F}^{S} \omega_{S}$. Eq. (5.9) shows how the attitude matrix from the gyroscope is integrated,

$$
{ }^{F} \boldsymbol{T}_{g}^{S}={ }^{F} \boldsymbol{T}^{S}+{ }^{F} \boldsymbol{T}^{S}{ }_{F}^{S} \tilde{\boldsymbol{\omega}}_{S} \Delta t,
$$

where the sample period is $\Delta t=0.02 s$.

The sensor fusion is preformed using a complementary filter,

$$
{ }^{F} \boldsymbol{T}_{C F}^{S}=w^{F} \boldsymbol{T}_{g}^{S}+(1-w)^{F} \boldsymbol{T}_{a}^{S},
$$

where the gyroscope weight is $w=0.98$.

In this Thesis, the base is considered fixed. Indeed, in a real TVC system, the attitude of the base also requires estimation; however, this analysis is beyond the scope of this work.

\subsection{1. Attitude Estimation Validation}

The attitude estimation using the IMUs is validated for the gimbaled platform using the encoders data. The gimbaled platform has two encoders and two IMUs, the lower is $192 \mathrm{~mm}$ above the cardan center, and the upper one is 302 $m m$ above. In this procedure, the attitude matrix ${ }^{F} \boldsymbol{T}^{S}$ of the CF is from the gimbaled platform, instead of the matrix presented in the last section. The attitude estimation using the lower IMU has achieved the best result, as shown in Fig. 5.4 using $\theta$ and $\phi$ angle. 

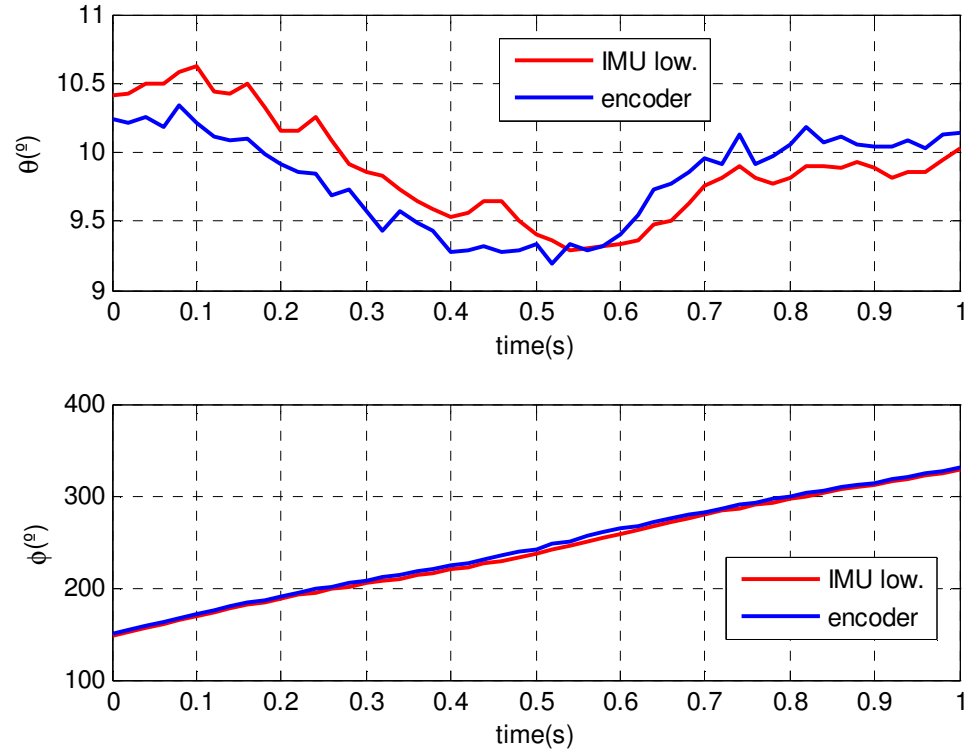

Figure 5.6: Attitude estimation validation with $\theta$ and $\phi$ angle.

The comparison using $\alpha$ and $\beta$ angle, depicted by Fig. 5.7, shows the error on the encoder axes.
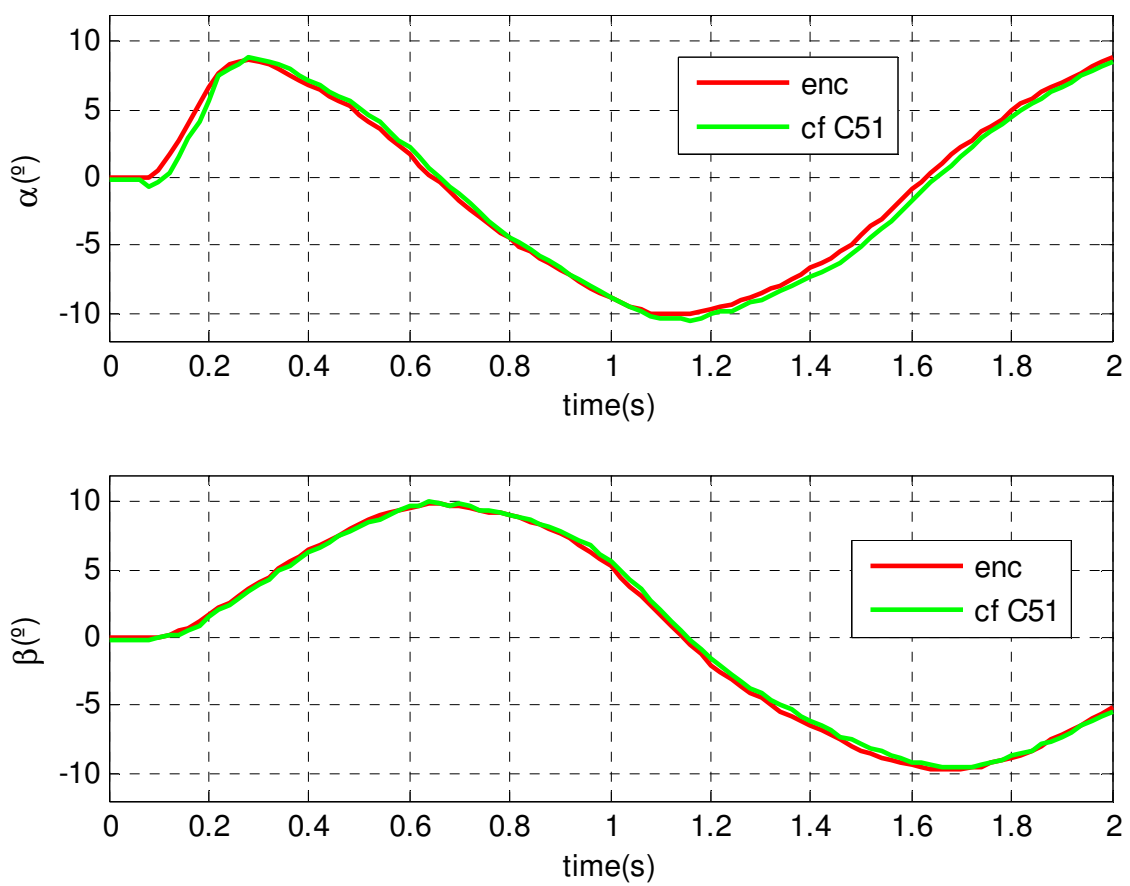

Figure 5.7: Attitude estimation validation with $\alpha$ and $\beta$ angle. 
The maximum absolute error in $\alpha$ during this test is $1.3^{\circ}$ and the mean $0.2^{\circ}$. In $\beta$ the maximum absolute error is $0.6^{\circ}$ and the mean is $0.1^{\circ}$. Thus, the attitude estimation has an average error close to the encoder resolution. Hence it is concluded that the attitude estimator is validated. However, it is important to highlight that this procedure considers only the short run of the system. During all tests, the platform starts in the vertical position, measured through a hand level. And before the controller starts to follow a trajectory, the platform is let stopped in this vertical position for $2 s$. The bias of the IMU gyroscope and accelerometer is corrected using these data. For the long run of the system, the bias of the sensors may vary and deteriorates the performance of the proposed system, but this is not evaluated in this work.

\section{5 .}

About the simulations

The controller algorithm is external to the numerical solution which simulates the discretization of the DAQ. The numeric solver time span is equal to the control period, hence is runs several times to accomplish the total simulation time. Figure 5.8 depicts the simulation loop.

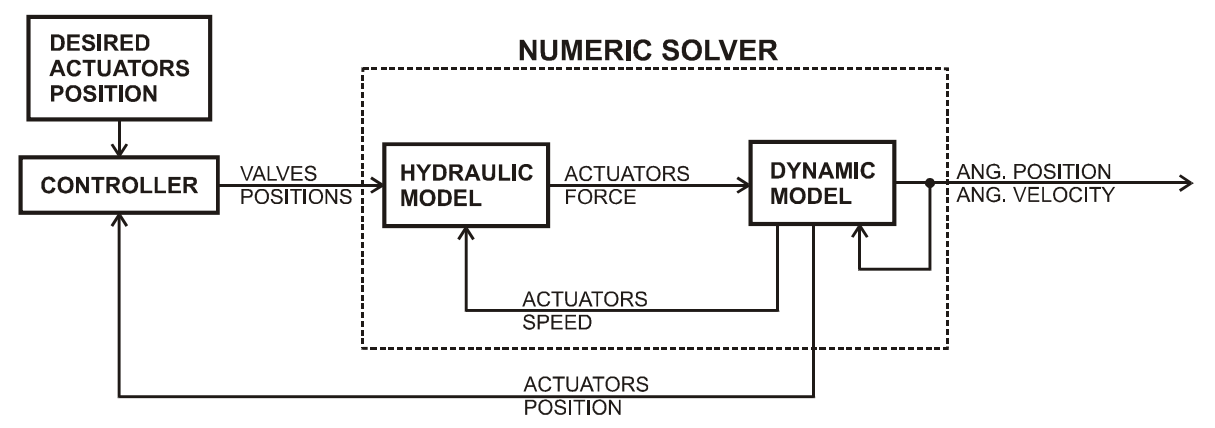

Figure 5.8: Simulation loop.

Using the valves position as input for the hydraulics model, the movement equation given by the dynamic model is integrated using the Matlab's solver ode $15 \mathrm{~s}$ with the maximum time step of $2 \mathrm{~ms}$. The ode 45 and ode 23 were also tried, but ode15s gives a similar accuracy with much smaller simulation time. The simulations of the next chapter use this simulation loop. 


\section{6 \\ Results}

In this section, the angular positioning accuracy of the gimbaled platform is initially evaluated. Then, the ability to follow trajectories is tested. The homokinetic platform is compared with the gimbaled platform for a particular desired trajectory. The performance of the gimbaled platform is also tested with various loads fixed on the moving table. Indeed, the aim of these tests is to observe the correspondence between the experiments and the numerical results. After ensuring the numerical reliability, other controller algorithms are tested through computer simulations. It saves time and avoids experiments with unstable controllers that can damage the system. Finally, the controllers with best performance are validated experimentally.

\section{1. \\ Gimbaled platform}

The experimental data are compared with the numerical simulation and the desired position. The trajectory is defined using articulation angle $\theta$ and angle $\phi$ defined as the angle between the -y-axis and the projection of $z^{\prime \prime}$-axis on the $x y$ plane. These angles are shown in Figure 6.1.

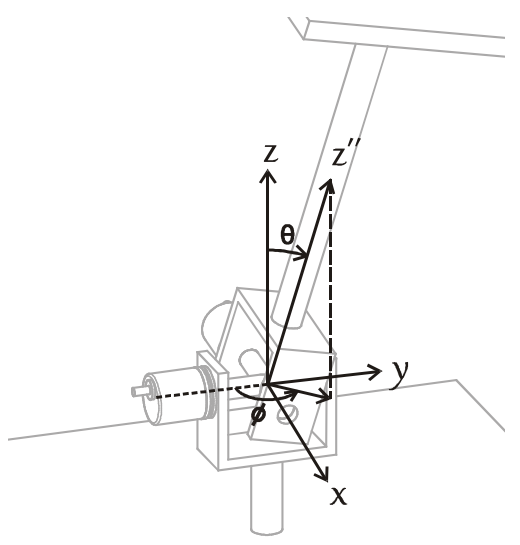

Figure 6.1: $\theta$ and $\phi$ definition 
These $(\theta, \phi)$ coordinates give the same orientation for the embarked $z$-axis in the homokinetic platform. Therefore, the comparison between the homokinetic and the gimbaled platforms is done using the same input trajectory. However, the rotation around the embarked z-axis is due to the kinematic constraint of the joint, which is different for the gimbaled and for the homokinetic platform.

Table 1 shows the parameters of the platform, load and controller. 
Table 1 - Parmeters

\begin{tabular}{|c|c|}
\hline Parameter & Value \\
\hline$l_{P}$ & $260.0 \cdot 10^{-3} \mathrm{~m}$ \\
\hline$m_{P}$ & $4.52 \mathrm{~kg}$ \\
\hline$I_{1 P}$ & $75.7 \cdot 10^{-3} \mathrm{kgm}^{2}$ \\
\hline$I_{3 P}$ & $16.9 \cdot 10^{-3} \mathrm{kgm}^{2}$ \\
\hline$l_{L}$ & $421.1 \cdot 10^{-3} \mathrm{~m}$ \\
\hline$m_{L}$ & $17.32 \mathrm{~kg}$ \\
\hline$I_{1 L}$ & $53.6 \cdot 10^{-3} \mathrm{kgm}^{2}$ \\
\hline$I_{3 L}$ & $45.8 \cdot 10^{-3} \mathrm{kgm}^{2}$ \\
\hline${ }^{F} \underline{g}$ & {$\left[\begin{array}{lll}0 & 0 & -9.81\end{array}\right]^{T} \mathrm{~m} / \mathrm{s}^{2}$} \\
\hline${ }^{S} \underline{r}_{C 31}$ & {$\left[\begin{array}{lll}-65 & 0 & 207.9\end{array}\right]^{T} \cdot 10^{-3} \mathrm{~m}$} \\
\hline${ }_{O}^{F} \underline{r}_{C 21}$ & {$\left[\begin{array}{lll}-135 & 0 & -30\end{array}\right]^{T} \cdot 10^{-3} \mathrm{~m}$} \\
\hline${ }^{S} \underline{r}_{C 32}$ & {$\left[\begin{array}{lll}0 & -65 & 207.9\end{array}\right]^{T} \cdot 10^{-3} \mathrm{~m}$} \\
\hline${ }_{O}^{F} \underline{r}_{C 22}$ & {$\left[\begin{array}{lll}0 & -135 & -30\end{array}\right]^{T} \cdot 10^{-3} \mathrm{~m}$} \\
\hline${ }_{O}^{F} \underline{r}_{C}$ & {$\left[\begin{array}{lll}0 & 0 & 354.3\end{array}\right]^{T} \cdot 10^{-3} \mathrm{~m}$} \\
\hline$k_{p}$ & $1.00 \cdot 10^{5}$ \\
\hline
\end{tabular}

The first trial for the gimbaled platform evaluates its proficiency to achieve a desired position.

\subsection{1.}

\section{Alternating between two positions}

In this case, the desired orientation of the platform alternates each second between $\left(\theta=15^{\circ}, \phi=0^{\circ}\right)$ and $\left(\theta=15^{\circ}, \phi=90^{\circ}\right)$, thus $\phi$ is a square wave with $45^{\circ}$ of amplitude and $1 \mathrm{~Hz}$ frequency and $\theta$ is constant. Figure 6.2 shows the numerical simulated (Sim) and the experimental (Act) result for $\theta$ and $\phi$ with the desired (Des) as reference. 

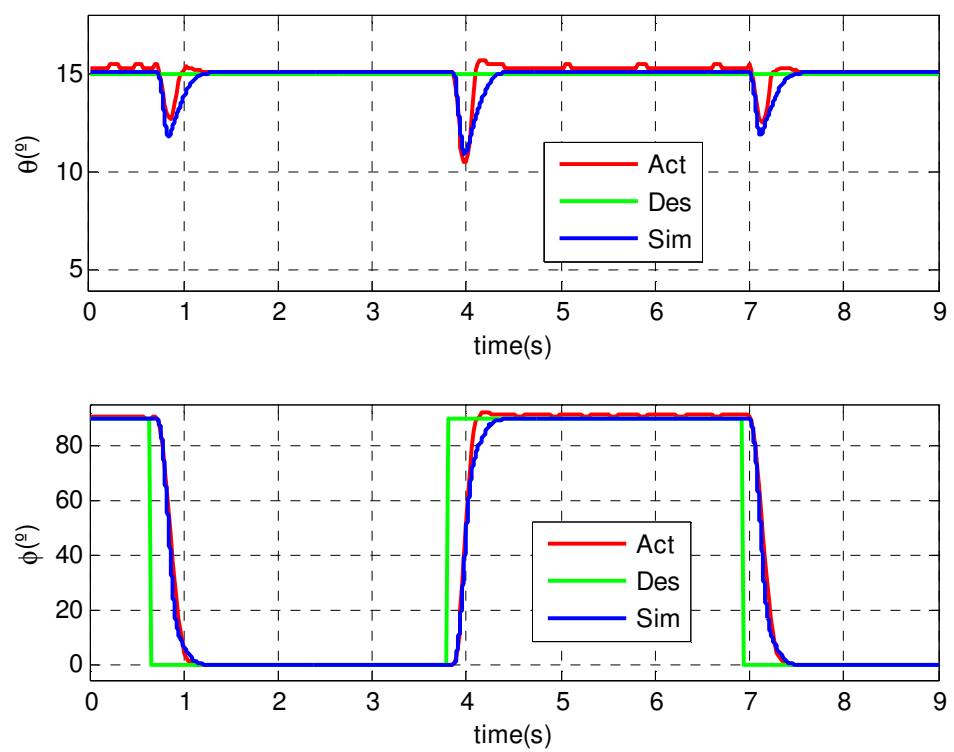

Figure 6.2: $\theta$ and $\phi$ result when alternating between two points.

The $\theta$ and $\phi$ results show a good correspondence between the experimental and the numerical result, although the actual result presents small overshoots. These angles are measured indirectly, using the encoders on the gimbal and the kinematic model of the system. Figure 6.3 shows results for the angles $\alpha$ and $\beta$.
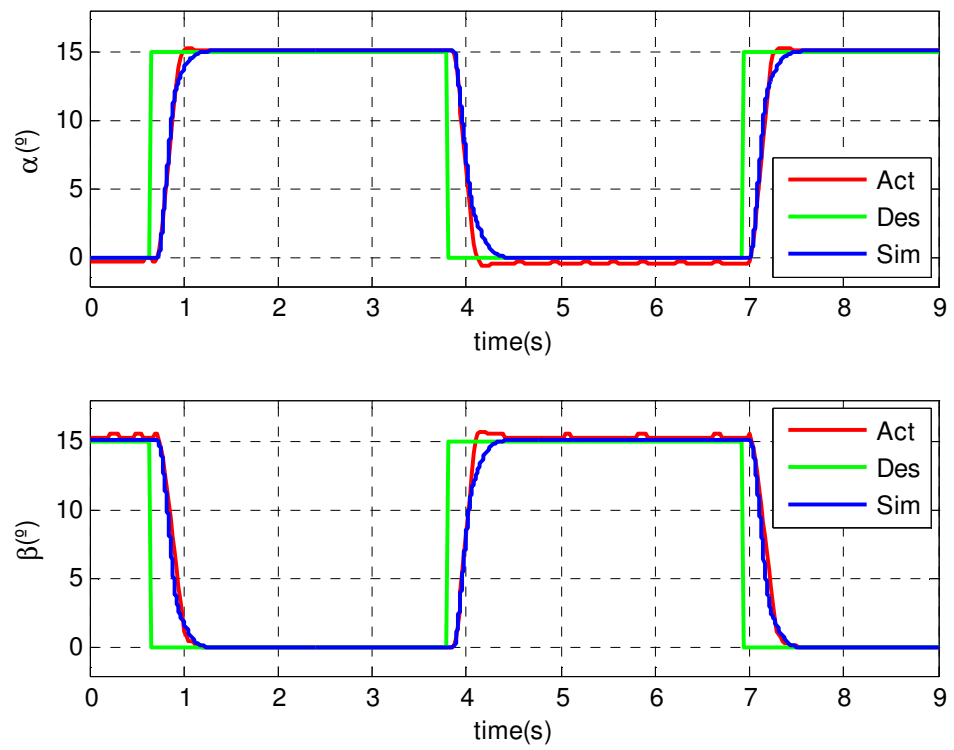

Figure 6.3: $\alpha$ and $\beta$ results when alternating between two points. 
The actual results of the angles $\alpha$ and $\beta$ are measured directly with the rotary encoder at the gimbal. Again, the numerical simulation presents a good insight about the behavior of the system and the actual $\alpha$ and $\beta$ have only small overshoots. After the settlement time, the $\alpha$ and $\beta$ deviation from the desired value is less than $0.36^{\circ}$, which corresponds to two increments of the encoder. During most of the time, the angular accuracy is lesser than the encoder increment. Hence, the final platform position accuracy is close to the best possible value allowed by these encoders. Figure 6.4 shows the results for the actuators length.
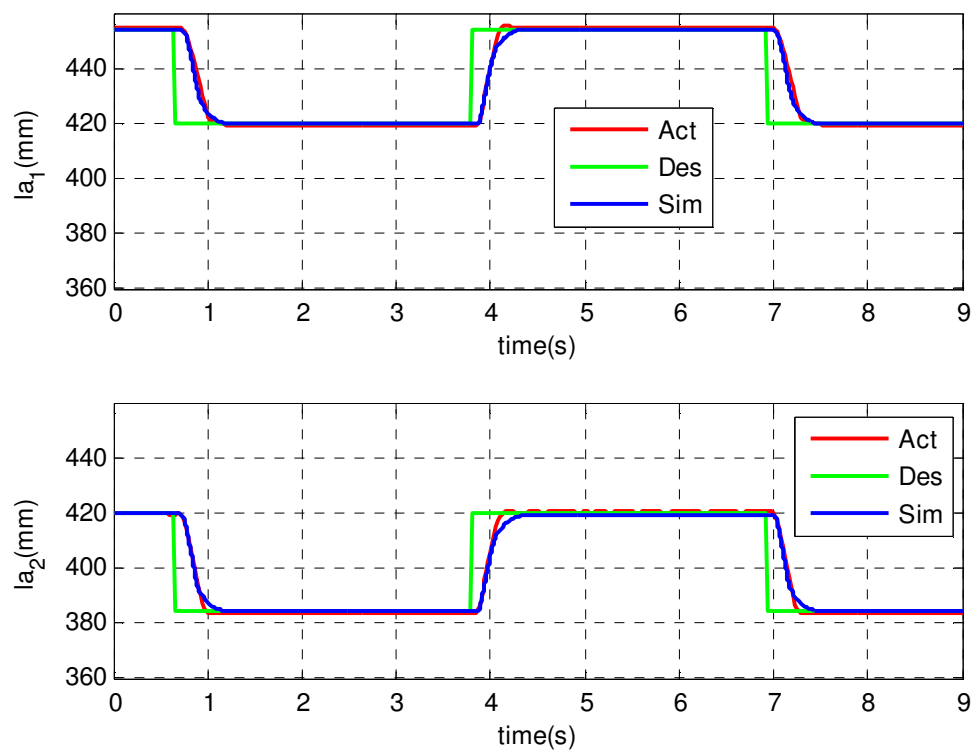

Figure 6.4: Actuators length when alternating between two points.

In this figure, the plot limits correspond to the maximum stroke of the actuators. The final position error is $0.5 \mathrm{~mm}$ in the average. The error in the controller is equal to the error in the actuators length. Indeed, the decentralized controller works like two independent controllers that receive the deviation of the actuators length from the desired position. Figure 6.5 shows the force done by the actuators. 

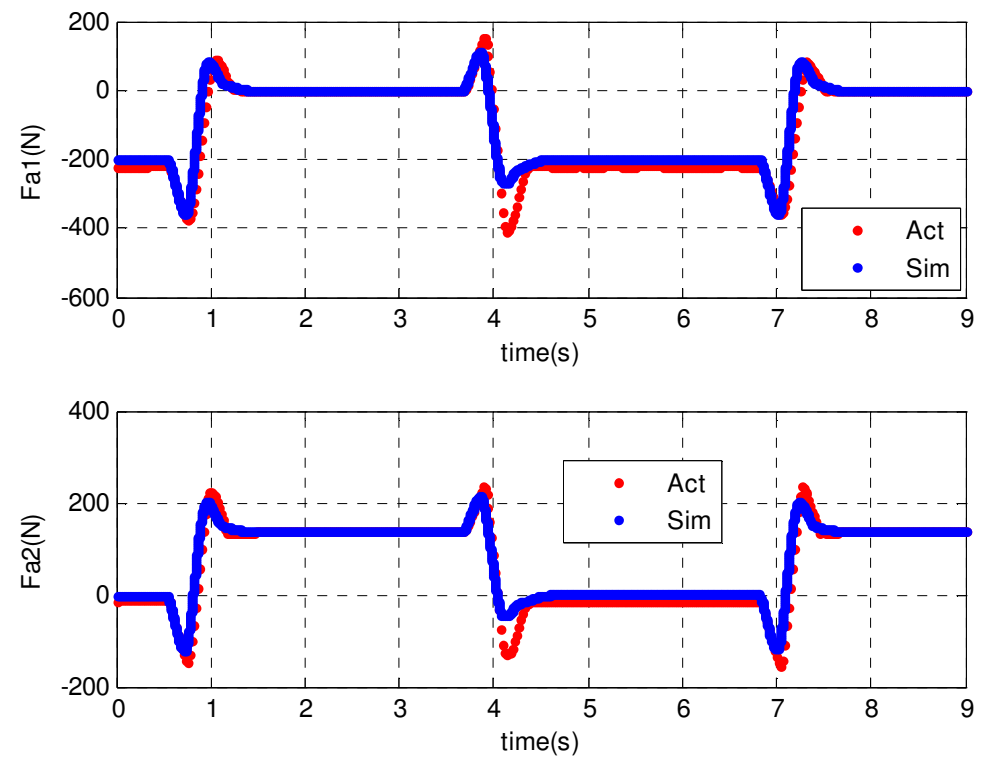

Figure 6.5: Actuator force when alternating between two points.

Once again, there is a good correspondence between the actual and simulated results. However, the small positioning overshoots allowed by the controller leads to bigger force peaks in the actual results. Figure 6.6 shows the controller outputs.
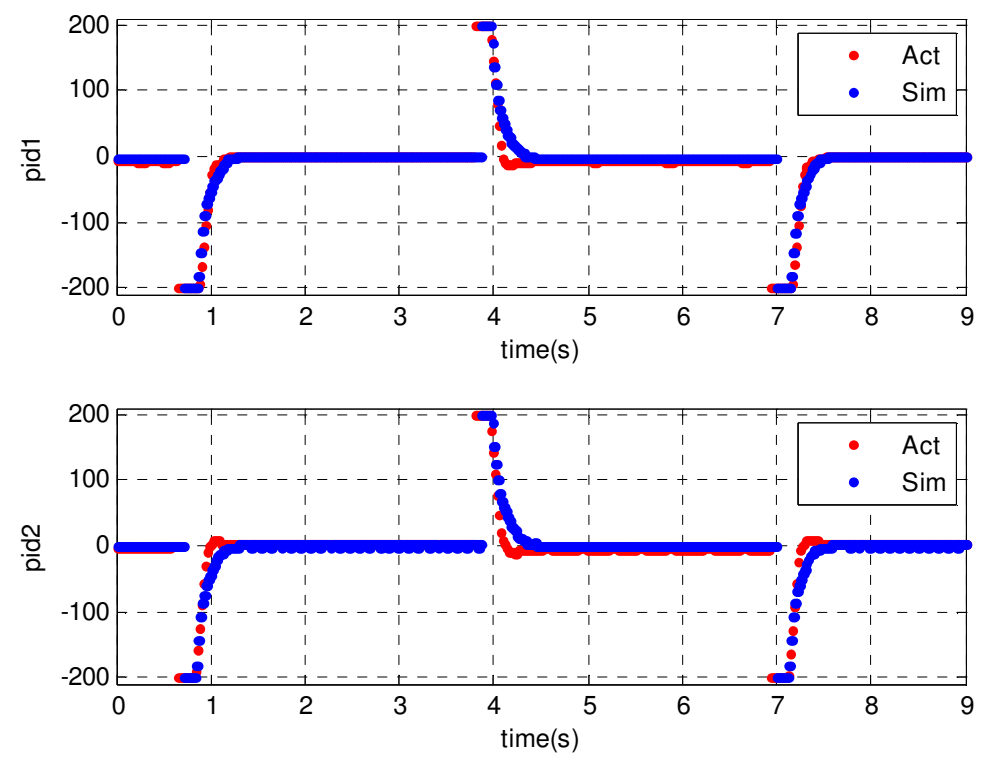

Figure 6.6: Controller output when alternating between two points. 
The absolute maximum controller output is 200 , which corresponds to the angular displacement of $14.4^{\circ}$ from the valve spool central position. The angular position of the valve can be positive or negative depending on the desired direction. The controller output demands full opening of the valve just after the change of the desired position. Figure 6.7 shows a top view of the table center trajectory.

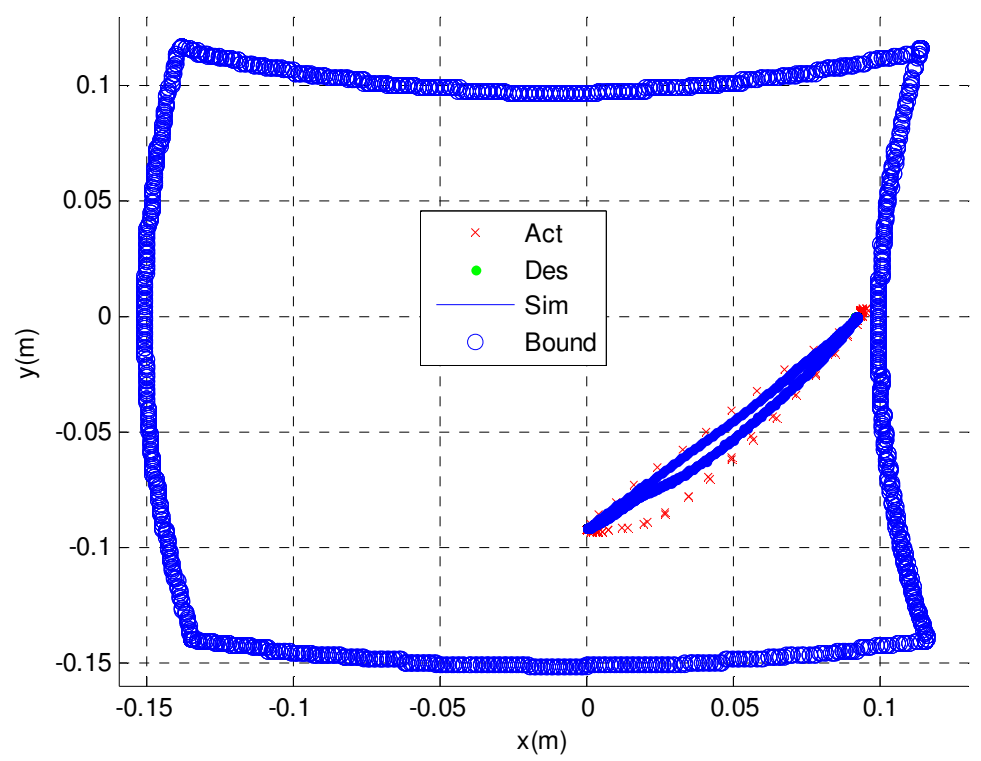

Figure 6.7: Table center trajectory when alternating between two points.

The blue circles in this figure define the working space of the platform. The table center position is limited to the maximum stroke of the linear hydraulic actuators. This graph reveals that the trajectory of the platform center is close to a straight line when it travels from the position closer to the boundary to the other position. This results as a peculiarity of the decentralized controller with the hydraulic actuators.

This case shows that the gimbaled platform is able to achieve a desired position with accuracy and stability. The next case evaluates the ability of the system to follow a desired trajectory. 


\subsection{2.}

Tricuspoid

In this case, the desired position of the table center projected on the $x y$-plane is a tricuspoid. Figure 6.8 shows a top view of the table center trajectory.

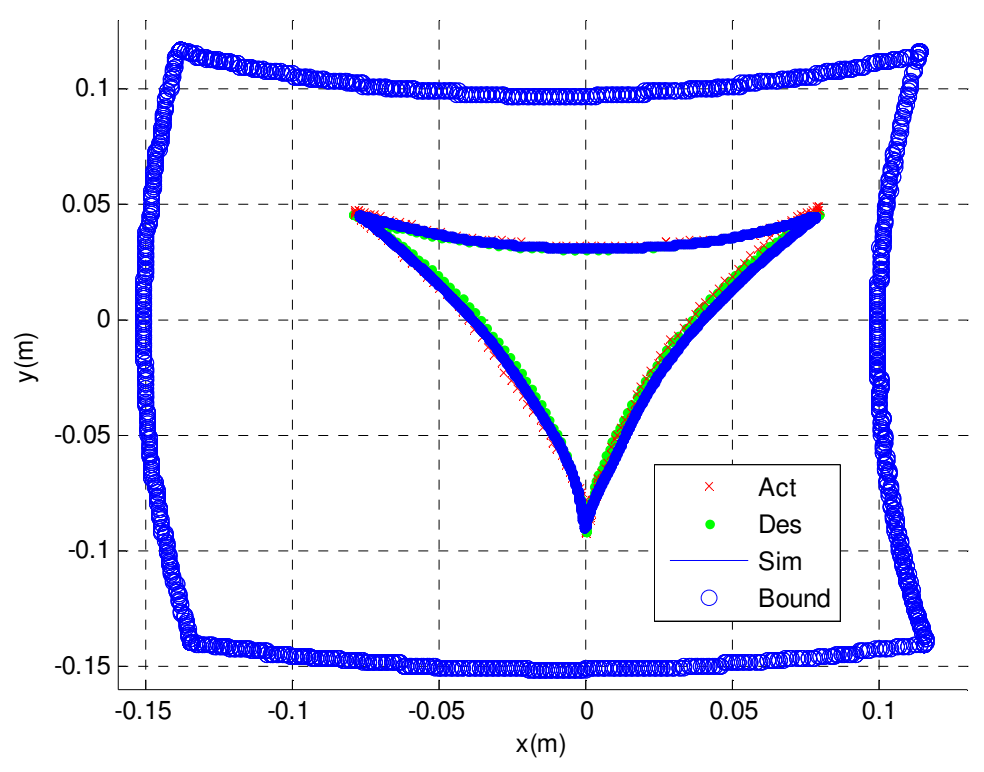

Figure 6.8: Table center trajectory for the tricuspoid.

The actual and the numerical simulated table center trajectory are close to the desired; however, the phase error cannot be seen in this plot. Fig. 6.9 shows the numerical and experimental results for $\theta$ and $\phi$. 

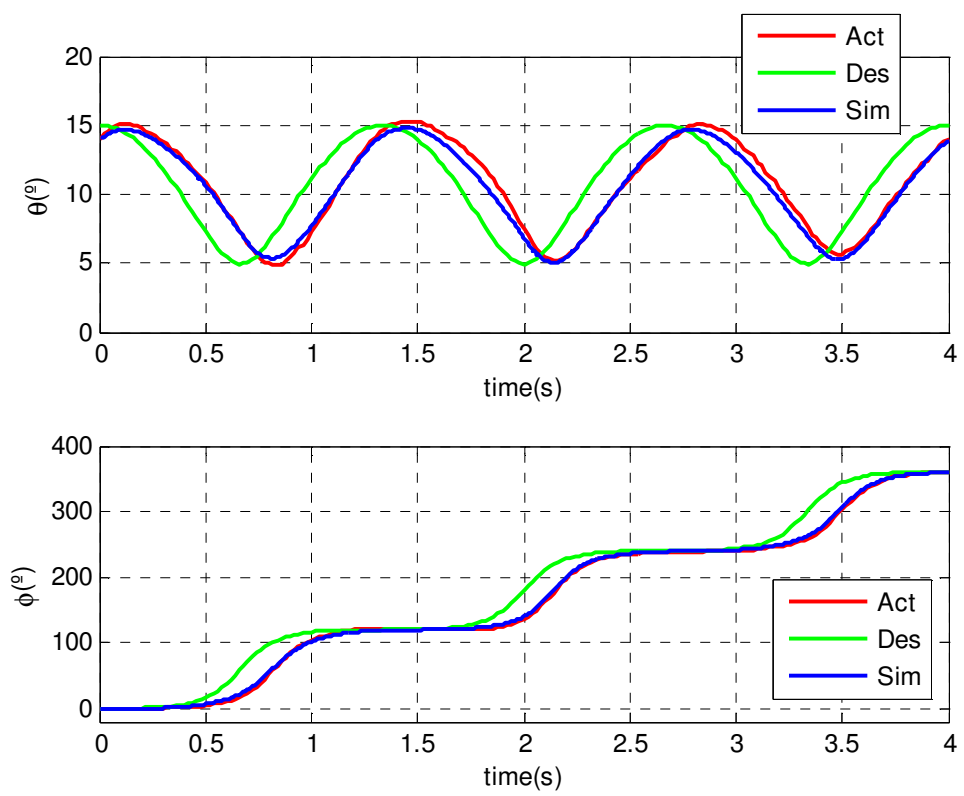

Figure 6.9: $\theta$ and $\phi$ results for the tricuspoid.

The $\theta$ and $\phi$ results show that the gimbaled platform performs the trajectory with a delay. This variable delay reaches $0.16 \mathrm{~ms}$ which corresponds to eight times the control loop period. Figure 6.10 shows results for $\alpha$ and $\beta$.
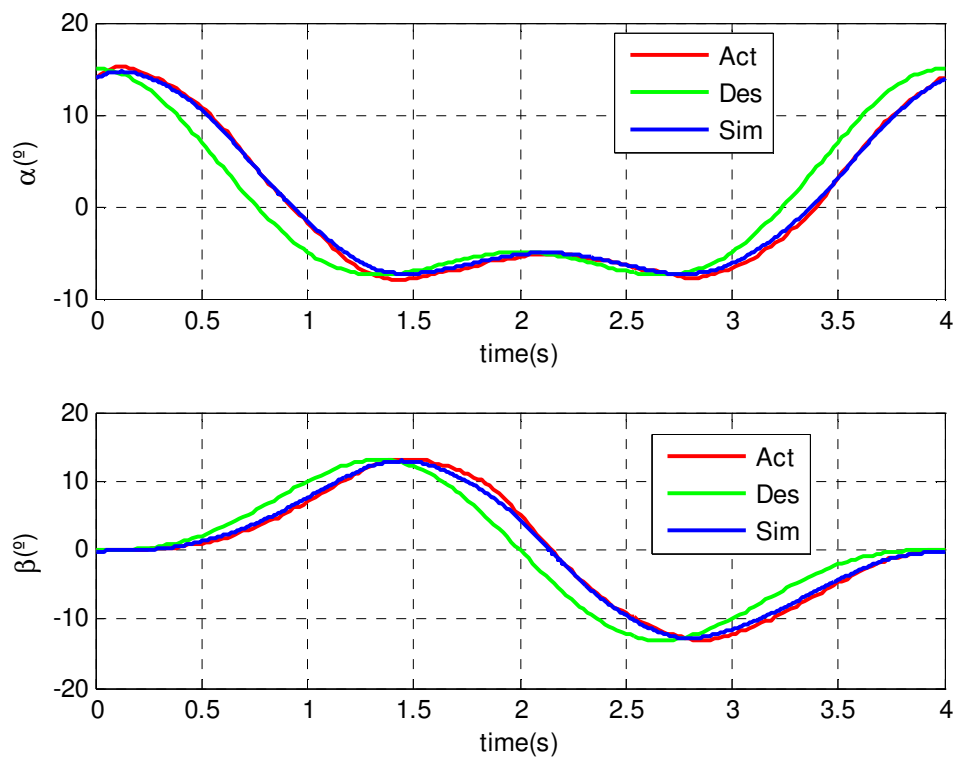

Figure 6.10: $\alpha$ and $\beta$ results for the tricuspoid. 
The numerical results of $\alpha$ and $\beta$ are close to the actual measurements with the encoders. Figure 6.11 shows the force done by the actuators.
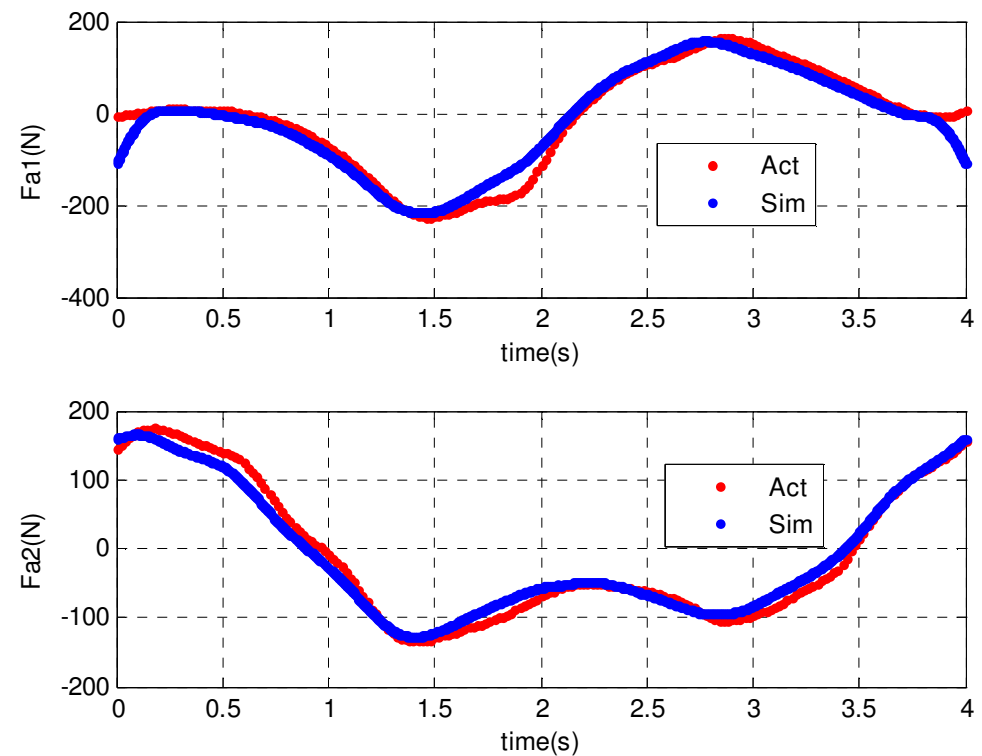

Figure 6.11: Actuator force for the tricuspoid.

The numerical and experimental results for the actuator forces allow the accuracy analysis of the dynamics modeling. In the experiments, the actuators forces are measured through load cells mounted on the piston rod. In the numerical simulations, the actuators inertia is considered negligible. Figure 6.12 shows the output control variable for this case.
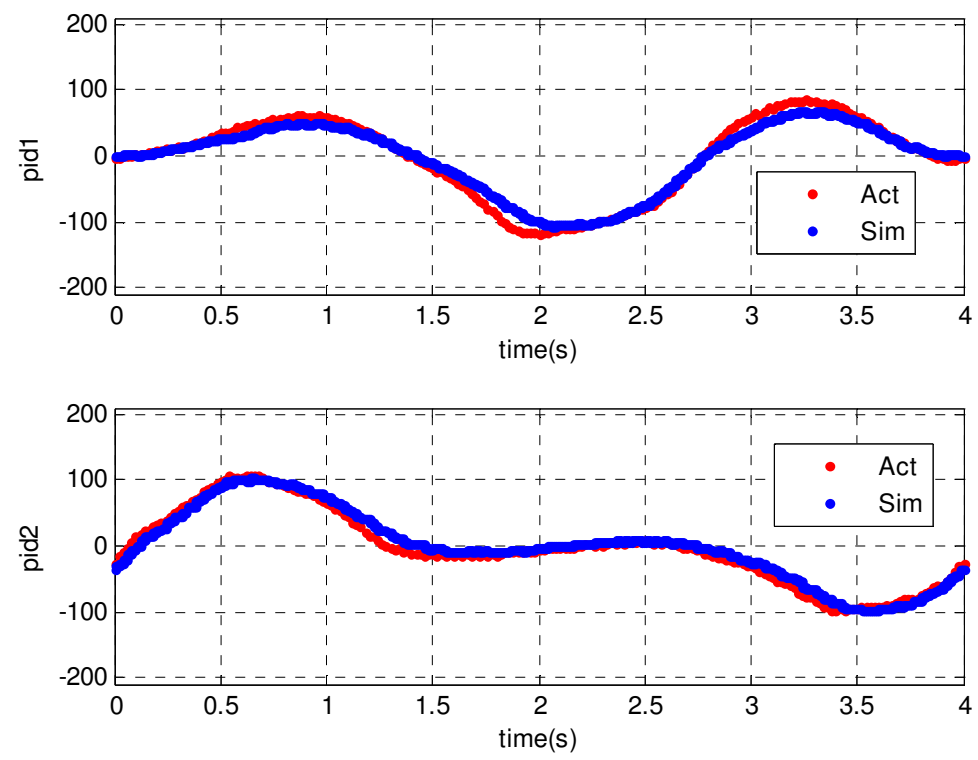

Figure 6.12: Controller output for the tricuspoid. 
The controller output shows that the valve opening during the experiment is close to $50 \%$ of the maximum. Figure 6.13 shows the actuators displacement.
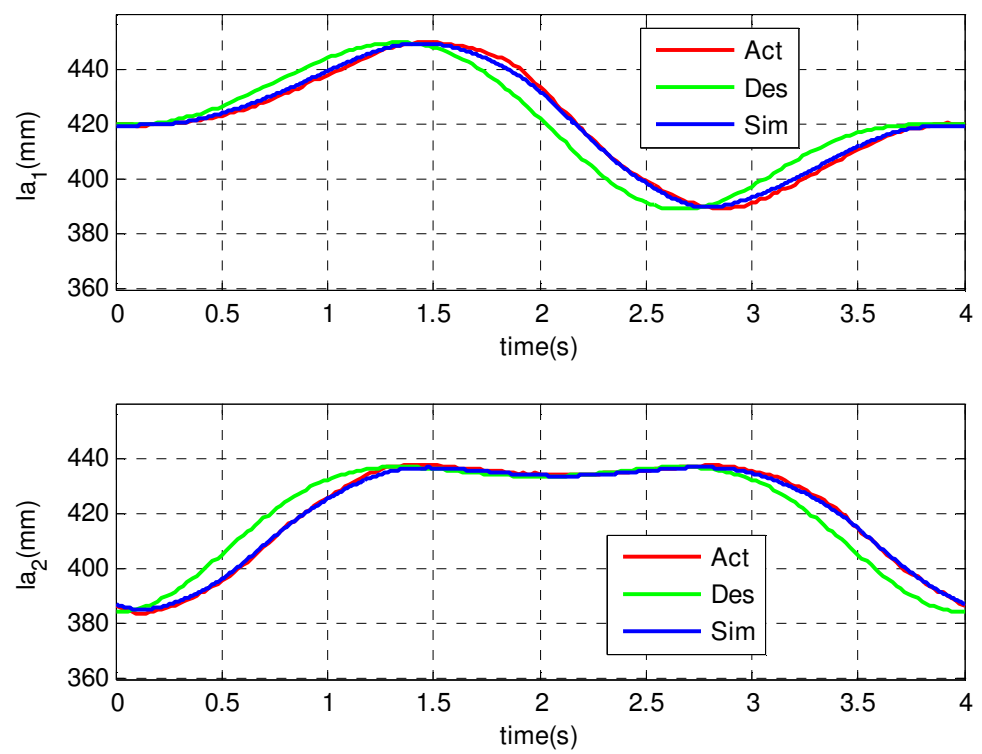

Figure 6.13: Actuators length for the tricuspoid.

These graphs reveal that the system is stable and able to follow the trajectory with precision, in spite of the time delay. The comparison between the actual results and the simulations indicates the consistency of the modeling. The next section evaluates the performance of the system for a simpler trajectory.

\subsection{3.}

\section{Circular trajectory}

In this case, the desired articulation angle is constant, $\theta=10^{\circ}$, and the $\phi$ angle changes with constant angular velocity $\dot{\phi}=\pi \mathrm{rad} / \mathrm{s}$ from 0 to $2 \pi$, thus the table center projection on $x y$-plane performs a circle. The top view of the table center trace can be seen in Figure 6.14 with the platform limits (Boundaries). 


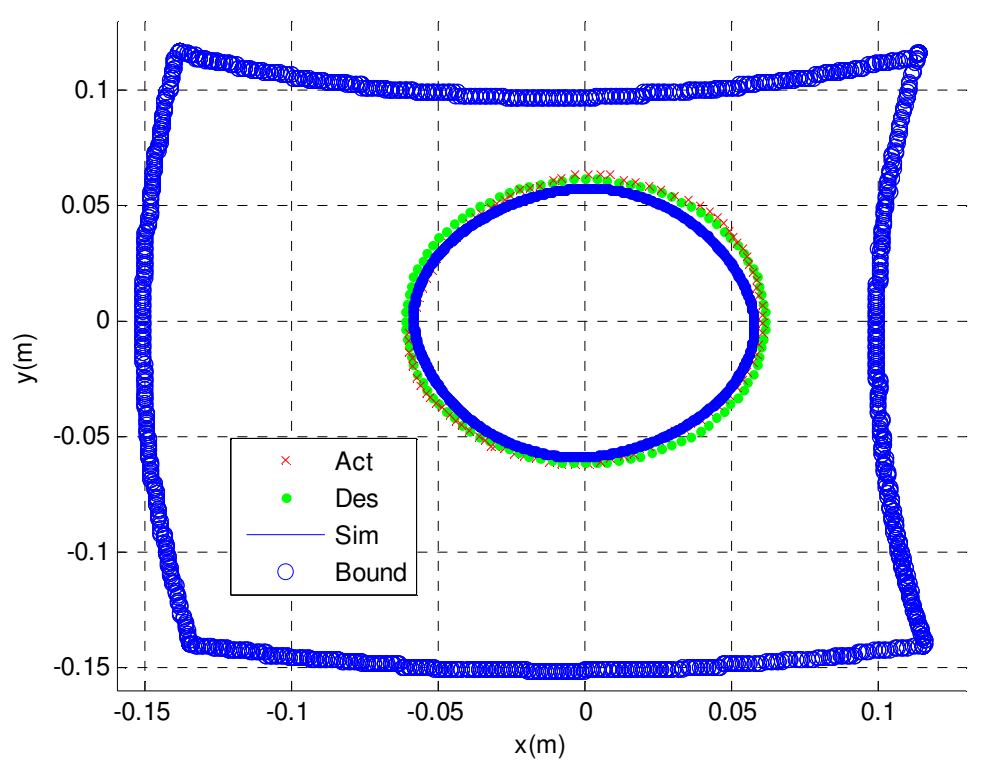

Figure 6.14: Top view of the table center trace for the circular trajectory.

In this figure, the upper right corner of the boundary, defined by the blue circles, will be achieved with the minimum length of the actuators. The lower left corner correspond to the maximum length of the actuators. The maximum divergence from the desired position is close to the minimum length of the actuator. Figure 6.15 shows the experimental results for $\alpha$ and $\beta$ comparing the numerical simulation and the desired positions.
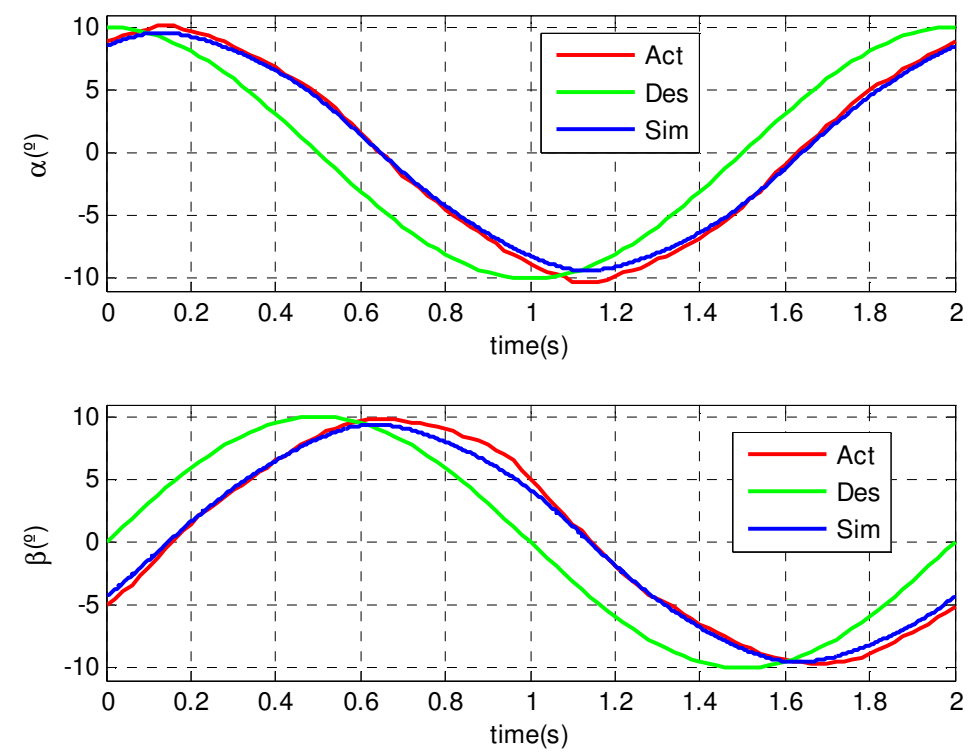

Figure 6.15: Platform attitude for the circular trajectory. 
The deviation between $\alpha$ simulated and the experimental result is $2,2^{\circ}$ in maximum and $1,2^{\circ}$ in average. The $\beta$ deviation is $1.8^{\circ}$ in maximum and $0.9^{\circ}$ in average. In this case, Figure 6.16 shows the actuators length where the plots limits are the actuator limits.
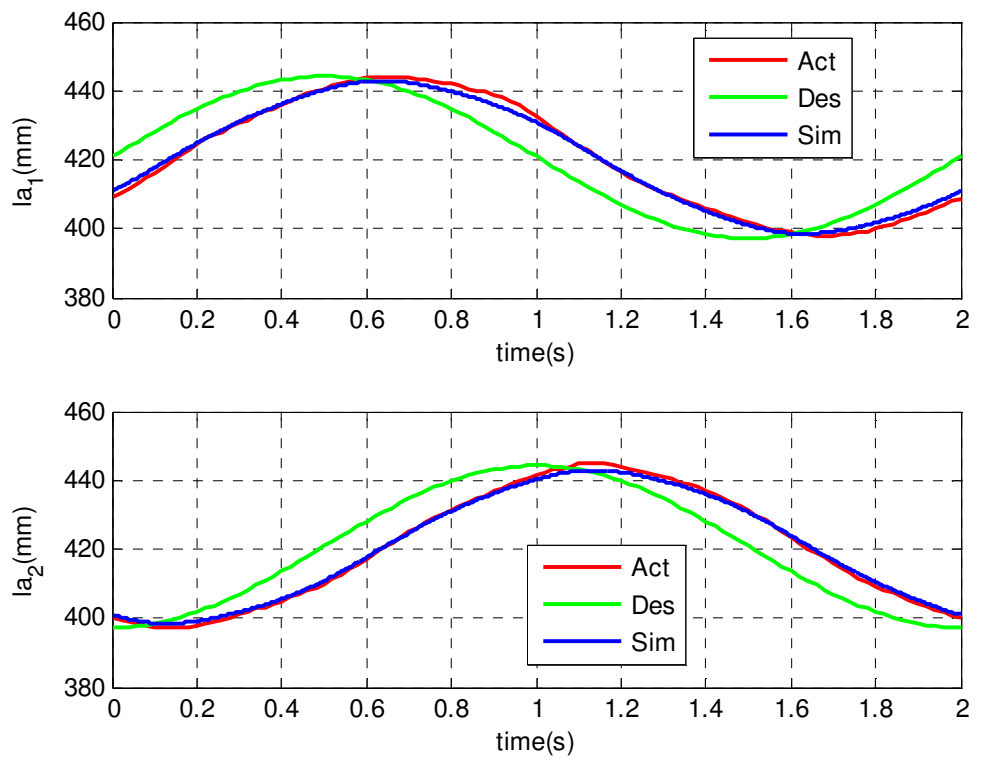

Figure 6.16: Actuators length for the circular trajectory.

The average error between simulated and the experimental actuators length results is $2 \mathrm{~mm}$, and this error achieves $4 \mathrm{~mm}$ for HA1 and $5 \mathrm{~mm}$ for HA2 in the maximum.

The difference between the actual (or the simulated) position and the desired position is mainly due the simplicity of the control algorithm. This proportional control with higher gain leads to stability problems, essentially chattering. The deviations between simulated and the experimental results are mostly due to model simplifications and platform assembly problems, like backlash and joint friction.

In the next section, another setup of the platform is tested: the homokinetic configuration. A method to compare both platform configurations is proposed. 


\section{2.}

\section{Homokinetic platform}

Similar to the gimbaled platform, in the homokinetic platform the experimental data are compared with the numerical simulations and desired position. The desired trajectory is the same circular trajectory demanded for the gimbaled platform.

Figure 6.17 shows the $\theta$ and $\phi$ angles for the experimental data compared with the numerical simulation and the desired position.
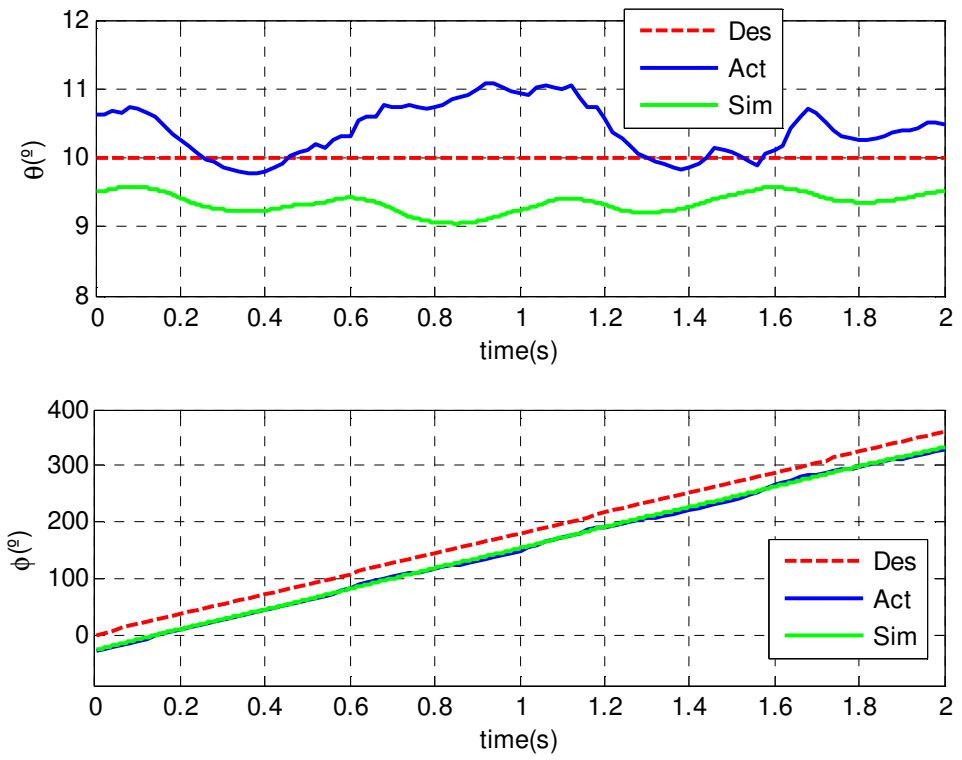

Figure 6.17: Simulated and experimental homokinetic platform orientation.

In this case, the $\theta$ and $\phi$ angles are estimated using the attitude estimation system presented in Chapter 5. The deviation between the simulated and experimental results for $\theta$ is $1.6^{\circ}$ at maximum and $0.8^{\circ}$ on average. The $\phi$ deviation is $2.8^{\circ}$ at maximum and $1.2^{\circ}$ on average. In this case, Figure 6.18 shows the actuators length, where the plots limits are the actuator limits. 

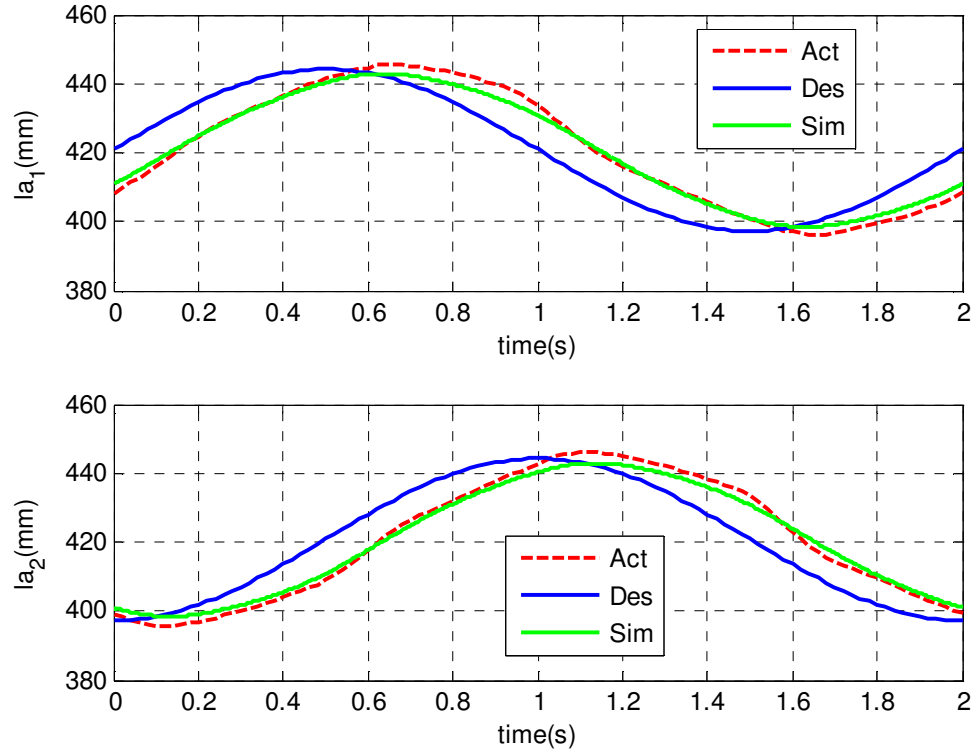

Figure 6.18: Homokinetic platform actuator length.

The average error between the simulated and the experimental actuator length results is $1.2 \mathrm{~mm}$, and this error reaches $2.2 \mathrm{~mm}$ for HA1 and $1.5 \mathrm{~mm}$ for HA2 at the maximum. The top view of the actual table center trace is shown in Figure 6.19 with the platform limits (Bound).

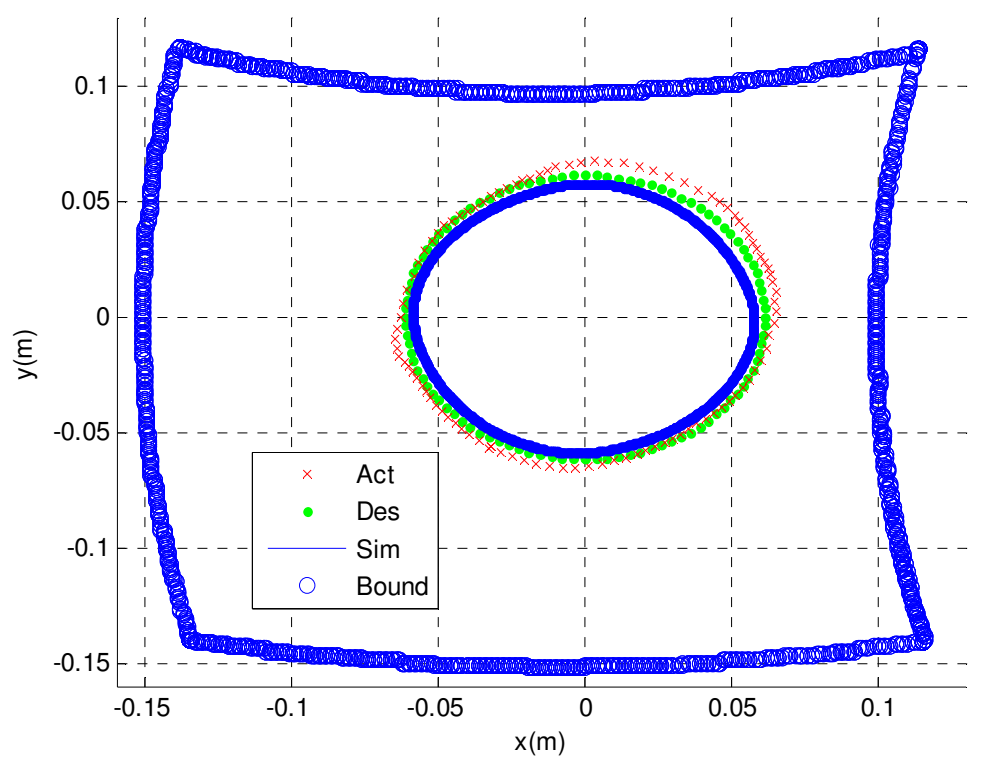

Figure 6.19: Top view of the homokinetic platform table center trace. 
As in the gimbaled platform, the simple P-controller limits the performance of the system and trying high gain leads to instabilities. The main cause of the deviation from the simulations is due to model simplification. Including friction losses in the pipelines, actuators inertia and a more realistic actuators friction model should reduce these differences.

\section{3.}

Comparison between gimbaled and homokinetic platform

In this section it is presented a comparison between the gimbaled platform and the homokinetic platform using the same conditions of the previous section. The position controller follows the same circular desired trajectory in both platforms. Hence the comparison begins showing in Fig. 6.20 the actual and the desired angular position of both platforms.
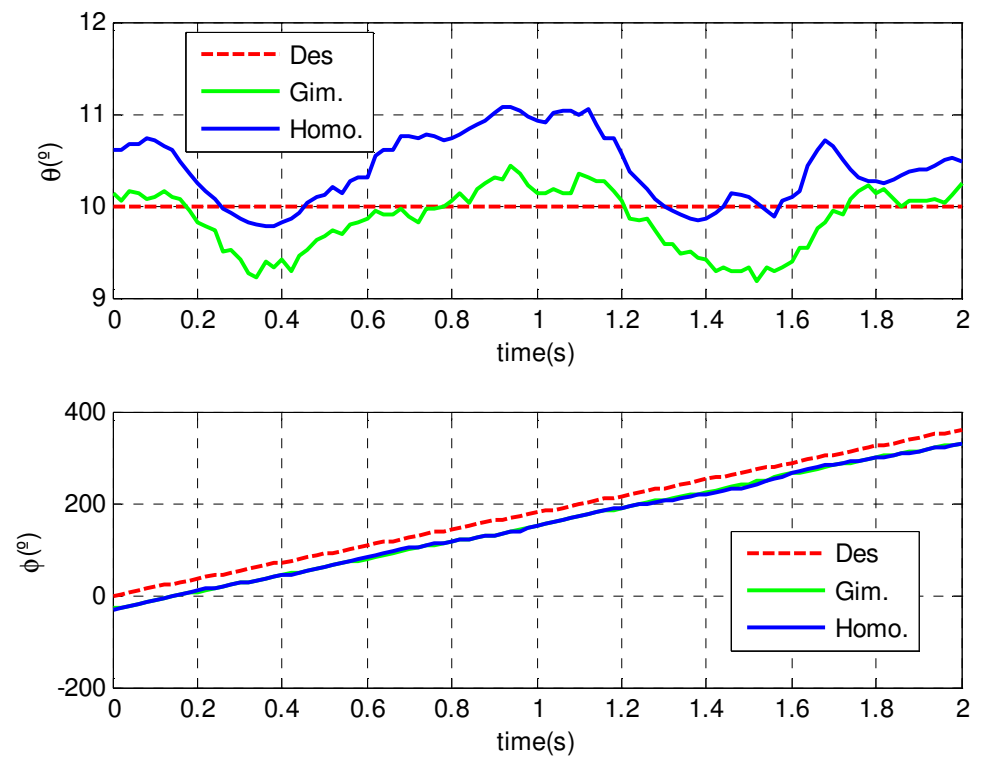

Figure 6.20: Attitude comparison between gimbaled and homokinetic platform.

For comparison purpose, the $\theta$ and $\phi$ angles of Fig. 6.20 are measured through the attitude estimation system based on an IMU as presented in Chapter 5 . Hence, the final comparison should consider the error of the attitude estimation system in the homokinetic platform. The gimbaled platform uses the encoders to measure the attitude, and its errors are significantly smaller as the estimated attitude using the IMU. The difference between actual and desired $\theta$ is about $0.5^{\circ}$ 
smaller in the gimbaled platform. And the error for the $\phi$ angle is $2^{\circ}$ smaller in the gimbaled configuration. Hence, the angular position tracking of the gimbaled platform is slightly better in this comparison using the attitude estimator for both platform configurations.

Through load cells on the rods the longitudinal forces generated in the actuators are measured. These values are compared with the simulated values for both platforms in Fig. 6.21.
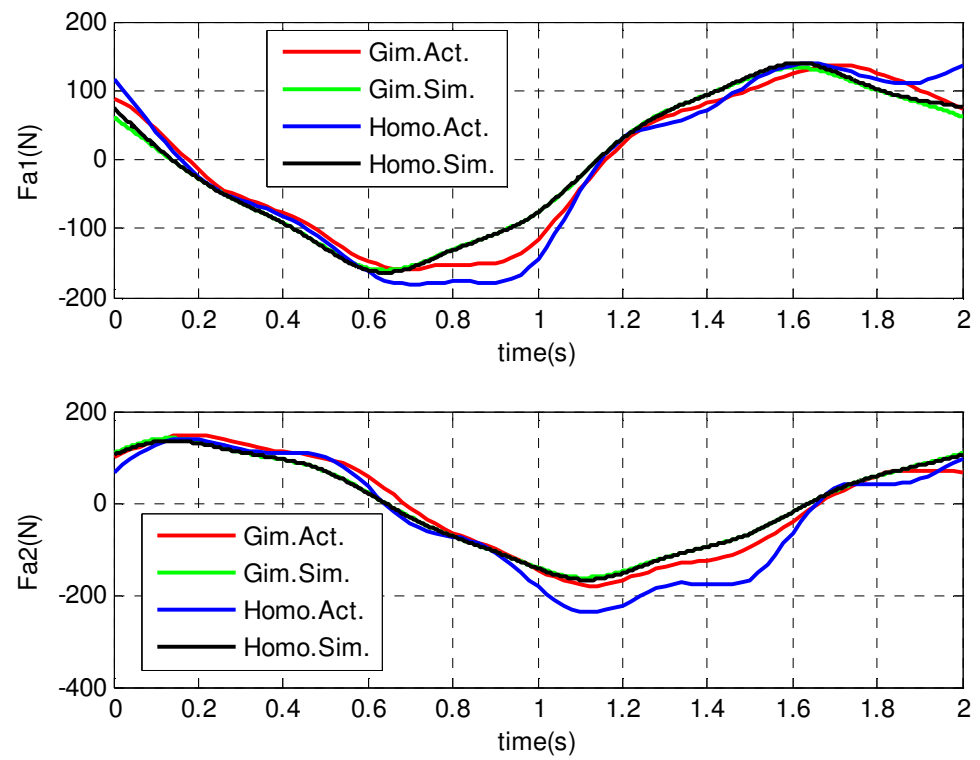

Figure 6.21: Force comparison between gimbaled and homokinetic platform.

There is a small difference between the gimbaled and the homokinetic platform. Hence, the actuator forces for both platforms are similar. However, the actual result for the gimbaled platform is closer to the simulated. Figure 6.22 presents the actual and simulated chambers pressure for the HA1 in both configurations. 

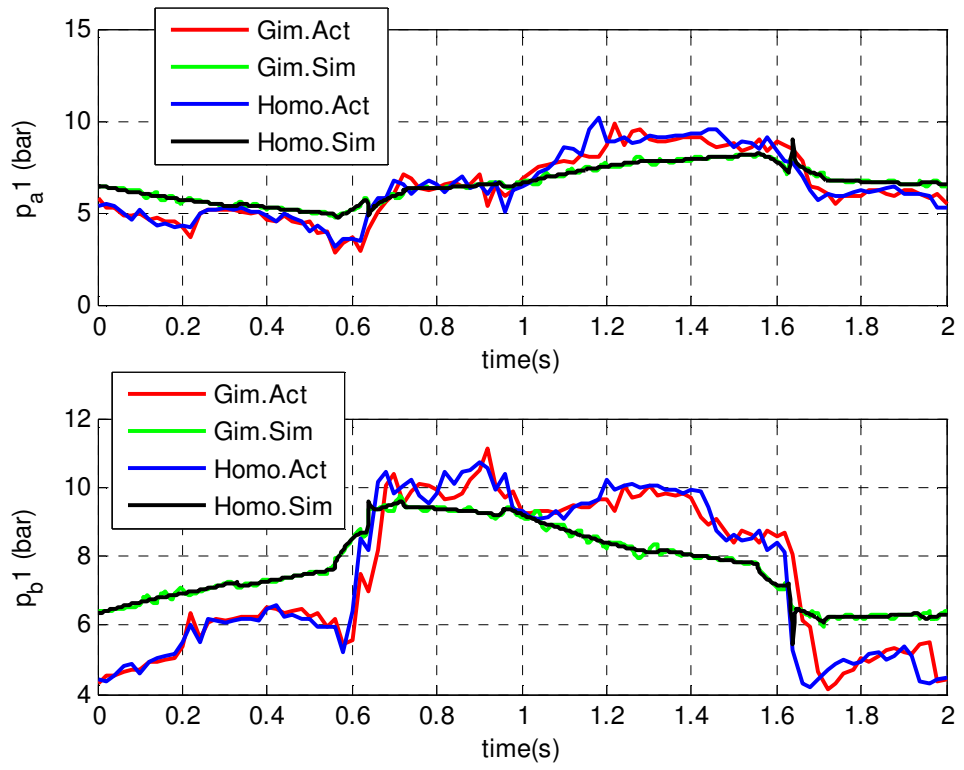

Figure 6.22: HA1 chambers pressure comparison.

Pressure transducers mounted directly at the actuator measures the chambers pressure. This result for HA2 is depicted in Fig. 6.23.
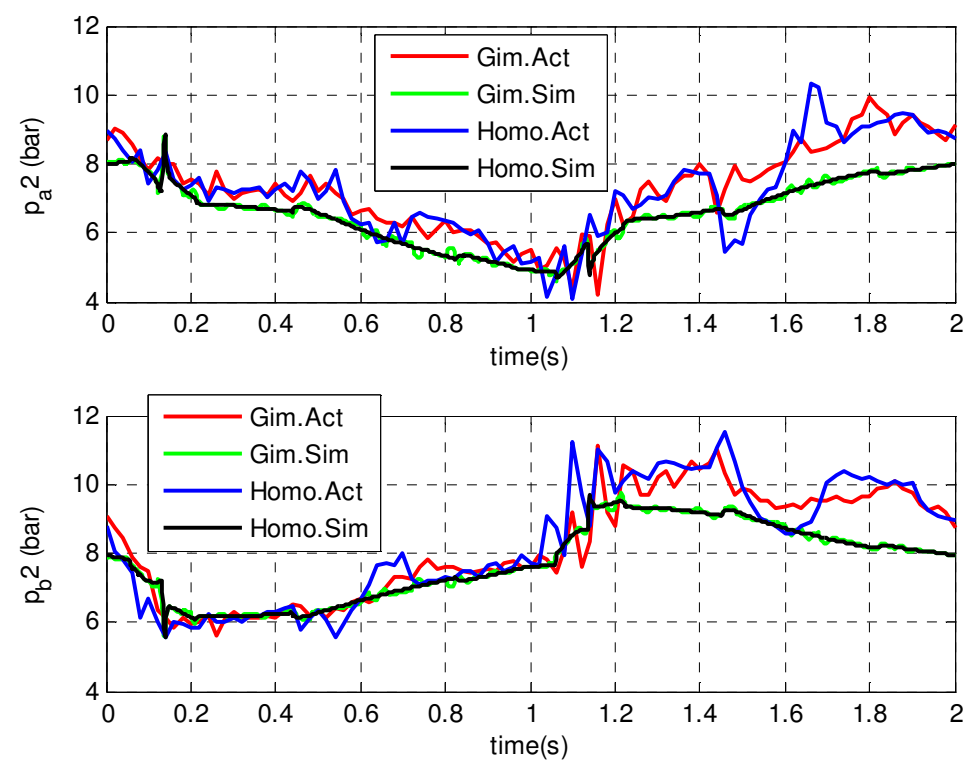

Figure 6.23: HA2 chambers pressure comparison.

The actuators chambers pressure in the gimbaled platform is similar to the pressure in the homokinetic platform. There is a difference between the simulated and the actual results. It is important to remember that the forces generated by the 
actuators are related to the area of the piston and the difference of pressure between the chambers. In the hydraulic model of the pipes, losses were not considered. Then, to achieve the same force in the actuator rod of the real system there is the necessity to reach higher pressure difference between the chambers. This explains the main part of the deviation of the numerical result.

\subsection{1. Spin rate comparison}

The angular velocity along the axis of the moving platform is called spin rate and it is different from the gimbaled version to the homokinetic version for the same table center trajectory. This difference is due to the different constraints of the gimbal when compared to the homokinetic joint. Different spin rates lead to different dynamics, thus a different moment acts on the fixed base. In this Thesis, the base of the platform is considered fixed. However, in a TVC system the base is attached to the rocket frame. The transversal moment in $x$ and in $y$ direction is balanced through TVC system itself. The longitudinal moment on the $z$ direction is controlled in single TVC system through aerodynamic devices or reaction rockets. As a consequence of the conservation of momentum, changes in the spin rate leads to torque on moving platform axis, which is transmitted through the joint to the rocket frame, or, in the analyzed platforms, to the fixed base. Figure 6.24 present a comparison between the gimbaled and the homokinetic platform spin rate using the numerical simulations data. 


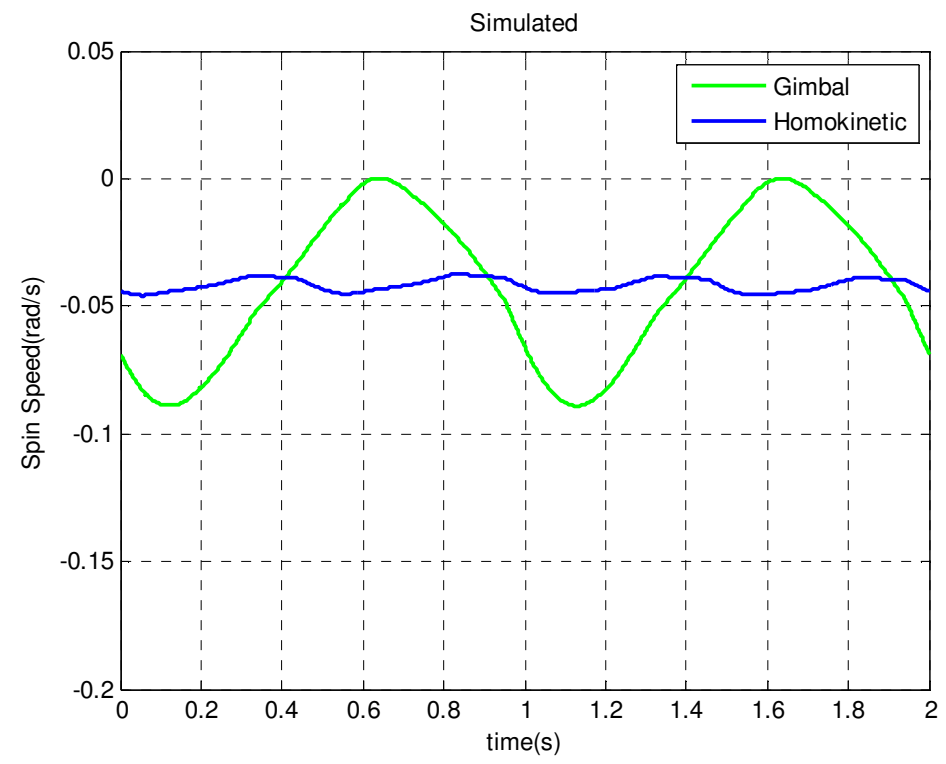

Figure 6.24: Numerical results for the platforms spin rate.

This result shows that for this trajectory, the spin rate in the homokinetic platform is better behaved. It is important to check if the joint behaves as predicted. Figure 6.25 shows the numerical results of the spin rate compared with the measured value using the IMU gyroscope.

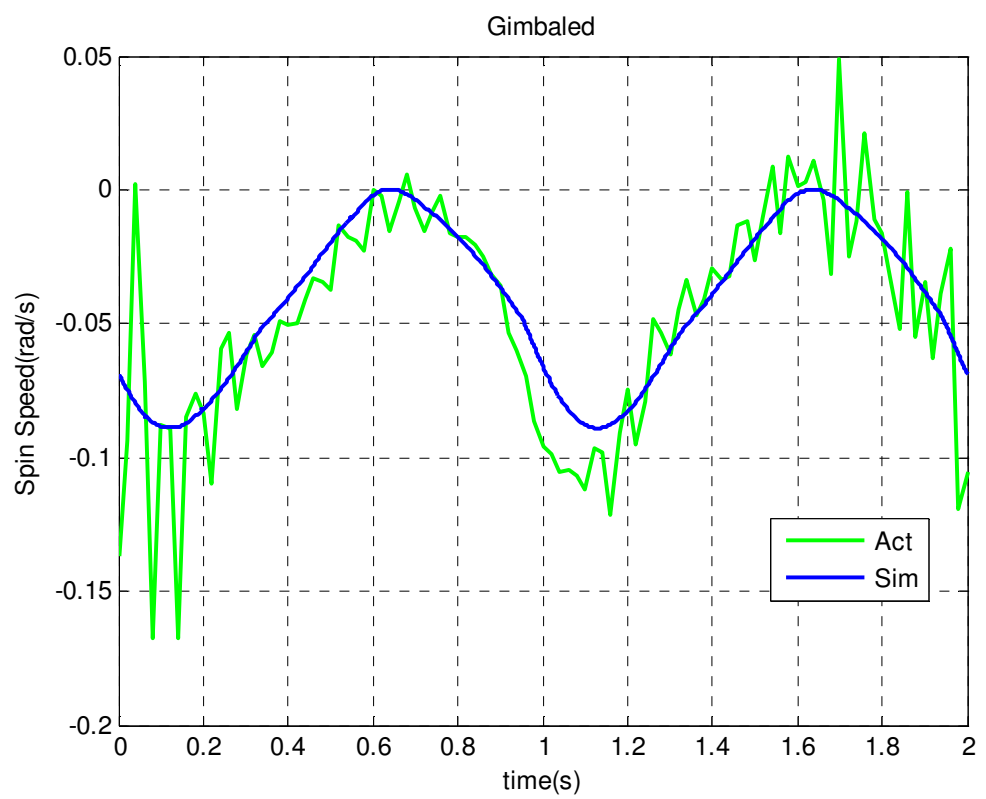

Figure 6.25: Gimbaled platform spin rate. 
This figure shows that the gimbal behaves as predicted, the difference is due to noise and backlash on several mechanical parts. Fig. 6.26 shows the results for the homokinetic moving table.

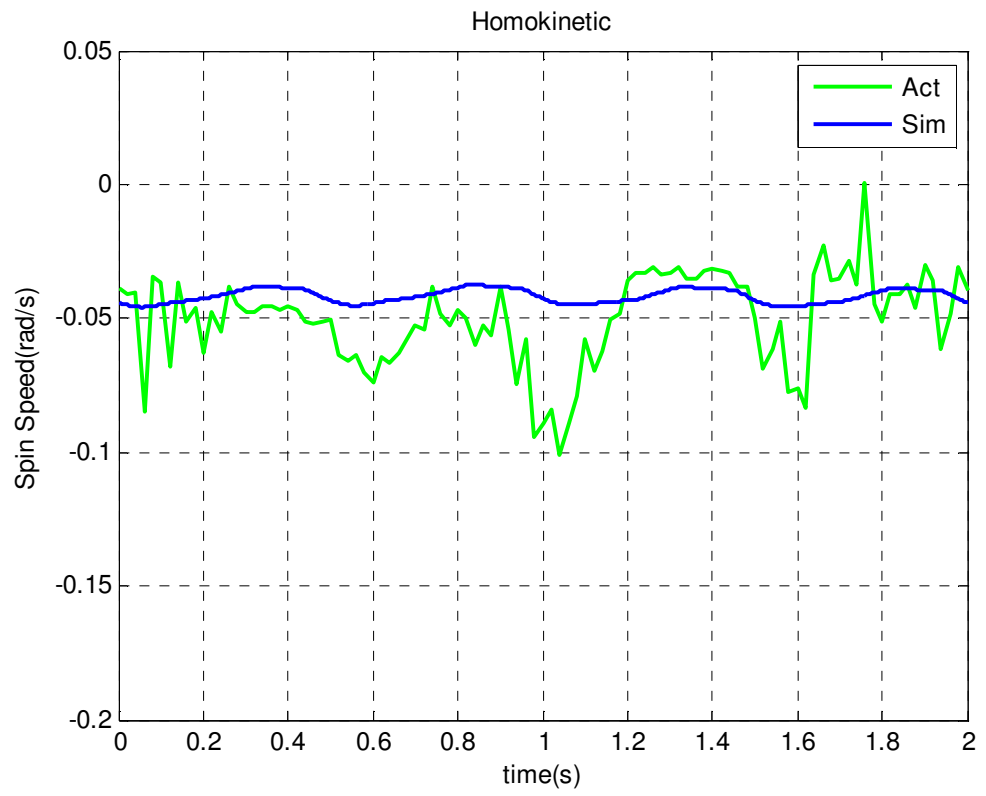

Figure 6.26: Homokinetic platform spin rate.

This comparison shows the similarities between experimental and numerical results, although the signal-to-noise ratio is higher than in the gimbal results. Indeed, the amplitude of homokinetic spin rate is smaller which leads to a poorer signal-to-noise ratio. And it is important to highlight that the CVJ used in this system is a rebuilt one and the gimbal was new. Hence, the improved performance of the homokinetic joint, showed by spin rate numerical results in Fig. 6.24, does not correspond to the experimental measurements. Indeed, Fig. 6.27 shows that the experimental homokinetic platform spin rate is not so much smaller, as predicted by numerical simulations. 


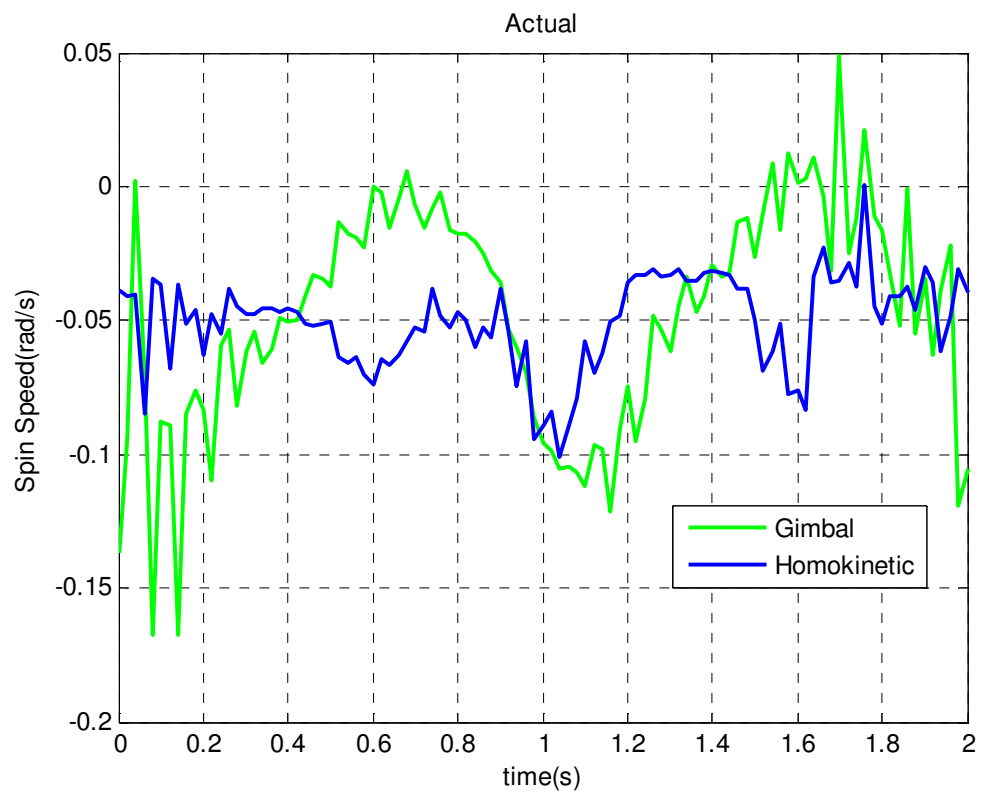

Figure 6.27: Experimental results for the platforms spin rate.

Experimental results show that the homokinetic platform does not have the entire improvement shown by the numerical results considering the spin rate. However, this is due to the low quality of the CVJ used and the low-cost sensors.

In spite of the simulations showing better behavior of the homokinetic platform spin rate, the analysis of the reaction moment on the base shows that this is not relevant. Figure 6.28 depicts the numerical results for the moment done by the base on the fixed z-axis for the gimbaled and the homokinetic platform for the circular trajectory of the table center. 


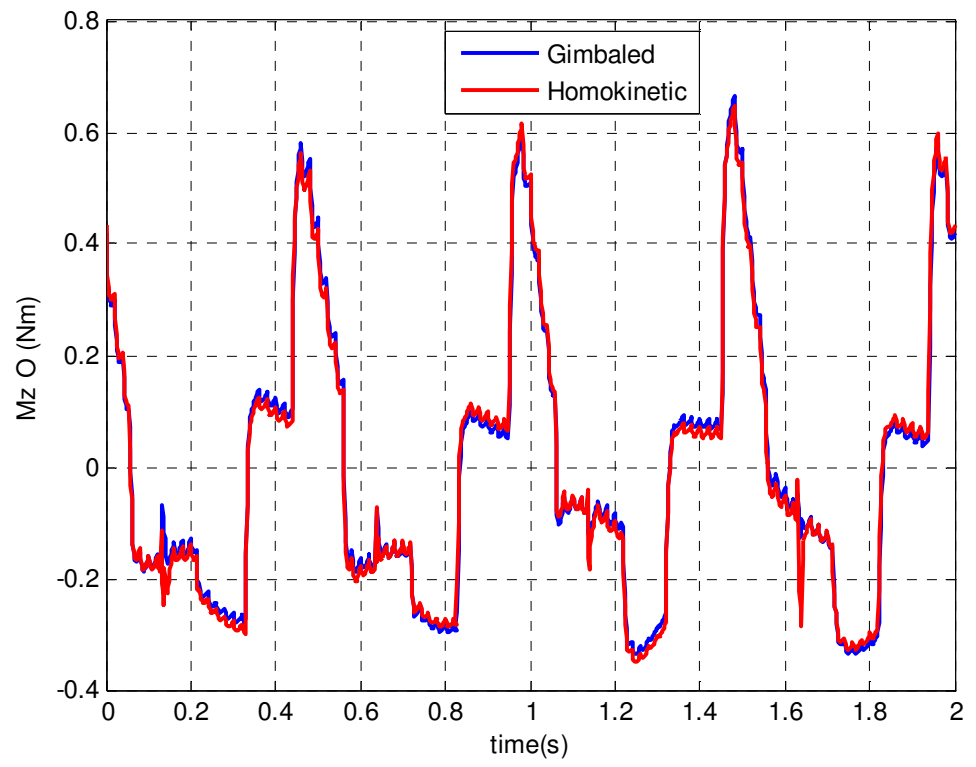

Figure 6.28: Moment done on $\mathrm{z}$ direction by the fixed base.

This figure shows that this moment is very similar for both platforms. Thus, the device responsible for rocket spin control requires similar power on both configurations. Unfortunately, there is no base torque transducer to validate this result experimentally. However, this moment is a consequence of the CVJ and the gimbal kinematics. Thus, the validation of the experimental embarked angular velocity data from IMU gyroscope assures that the gimbal and the CVJ behave as predicted. The angular velocity measured transversal to the moving table axis is a function of the trajectory, and the results are very similar for both platform configurations.

\section{4.}

Various load conditions on the gimbaled platform

In this section the results for three loads fixed to the moving table are considered:

- no load: There is no additional load over the moving table;

- load 1: The load is the same as in the cases presented above and shown in Table 1; 
- load 2: More load is added in this case. The load parameters changes to $l_{L}=482.8 \cdot 10^{-3} \mathrm{~m}, \quad m_{L}=31.67 \mathrm{~kg}, \quad I_{1 L}=234.5 \cdot 10^{-3} \mathrm{kgm}^{2} \quad$ and $I_{3 L}=80.1 \cdot 10^{-3} \mathrm{kgm}^{2}$.

The trajectory is the same circular trajectory presented in the last section. Figure 6.29 shows the actuators forces for these load conditions.
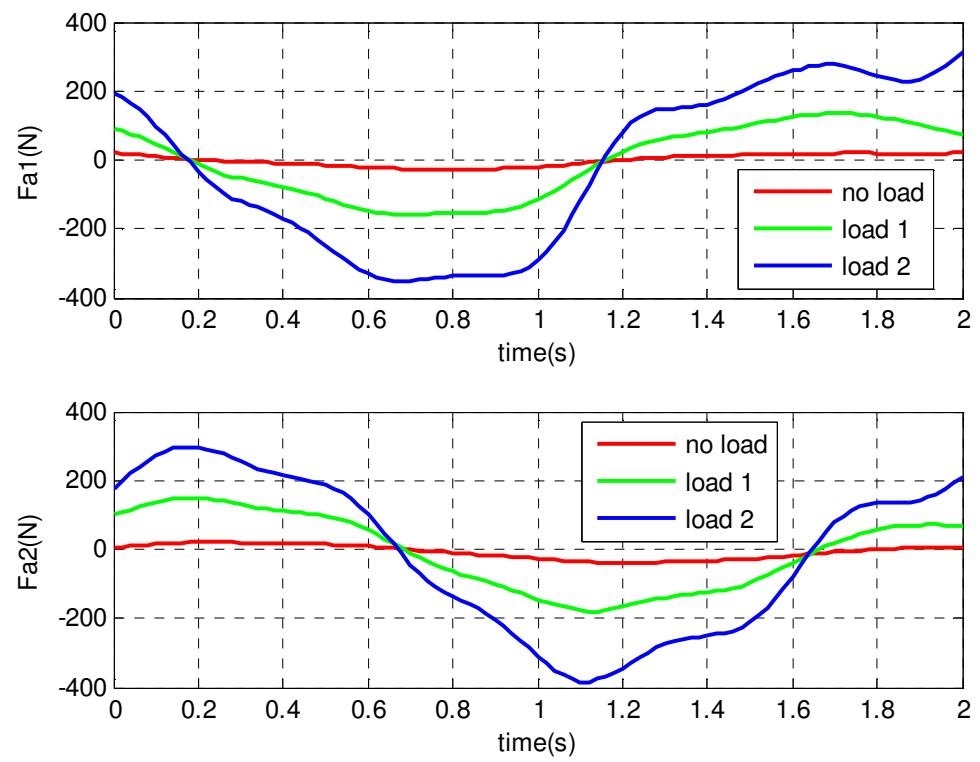

Figure 6.29: Actuators forces with different loads.

Hence, by the figure, from "no load" to "load 2", the force increases one order of magnitude. However, Fig. 6.30 shows that the controller output variable does not vary, thus the valve opening is almost the same for all load conditions. 

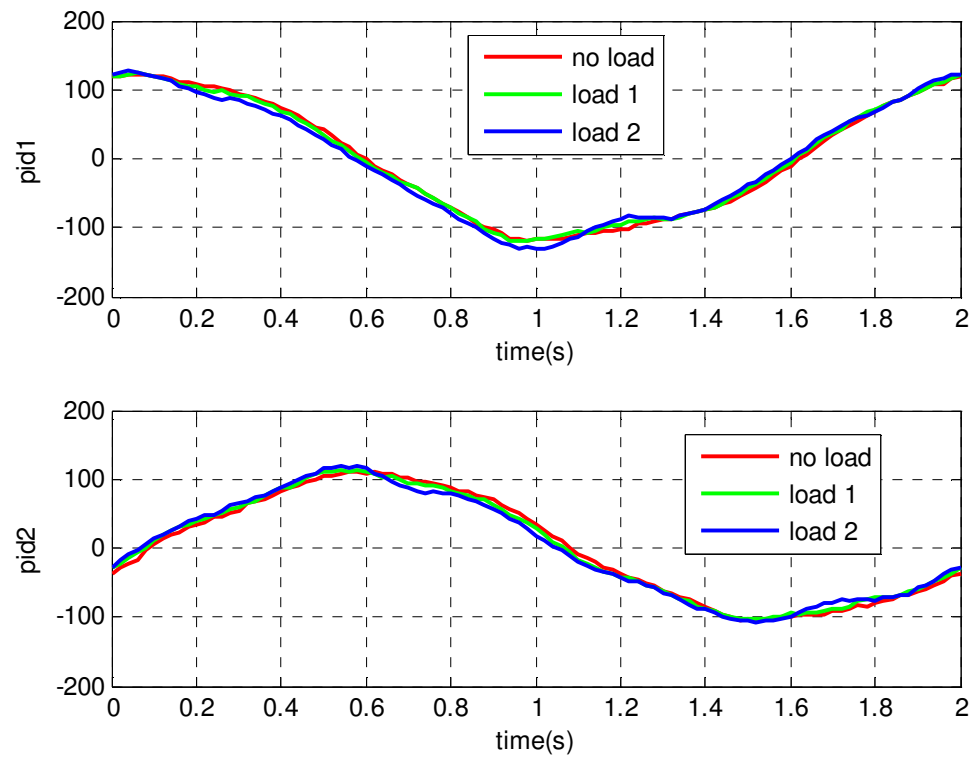

Figure 6.30: Controller output on different loads.

In spite of a force ten times bigger of the actuators and the same controller output, Fig. 6.31 shows that the displacement of the actuators is very similar, independent of the load.
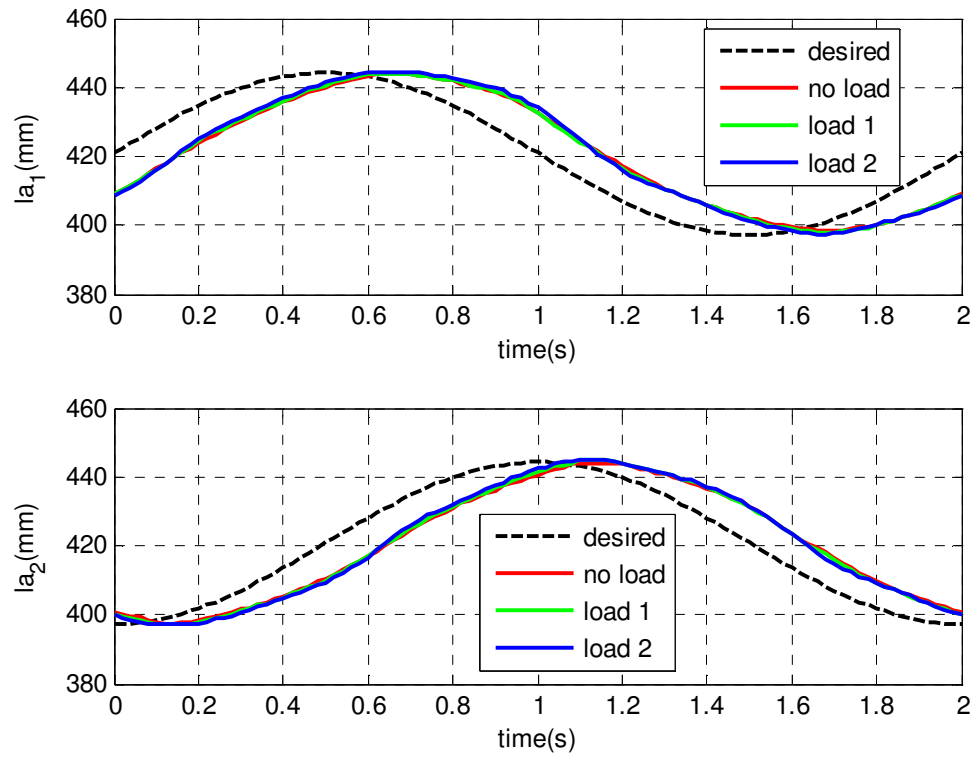

Figure 6.31: Actuators displacement on different loads.

These results reveal an insensibility of the hydraulic system to the load. This phenomenon happens in a limited force range. Since the "load 2" is the maximum 
payload of the platform, the next experiment increases the desired articulation angle to increase the actuators forces. In these experiments the desired articulation angle $\theta$ is $15^{\circ}$ during the circular trajectory of the table center, and the other parameters keep the same. Figure 5.32 shows the actuators forces in this condition.
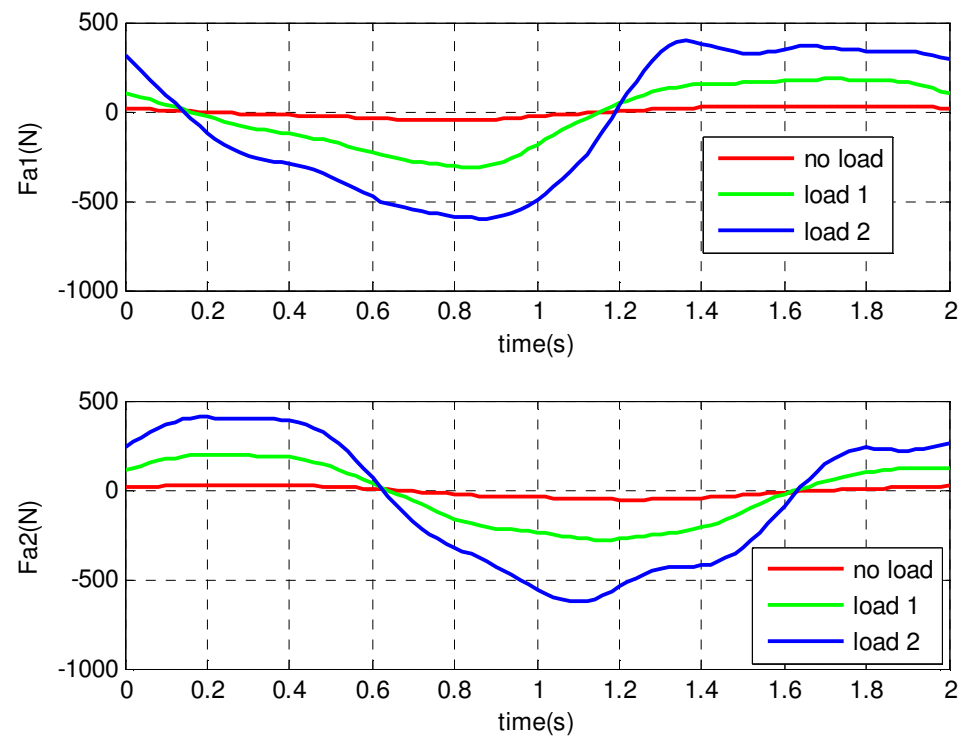

Figure 6.32: Force for different loads on a wider trajectory.

Figure 6.33 shows that in this case, the result of the controller output with the "load 2" increases, reaching the saturation on HA1 controller around the $t=1 \mathrm{~s}$.
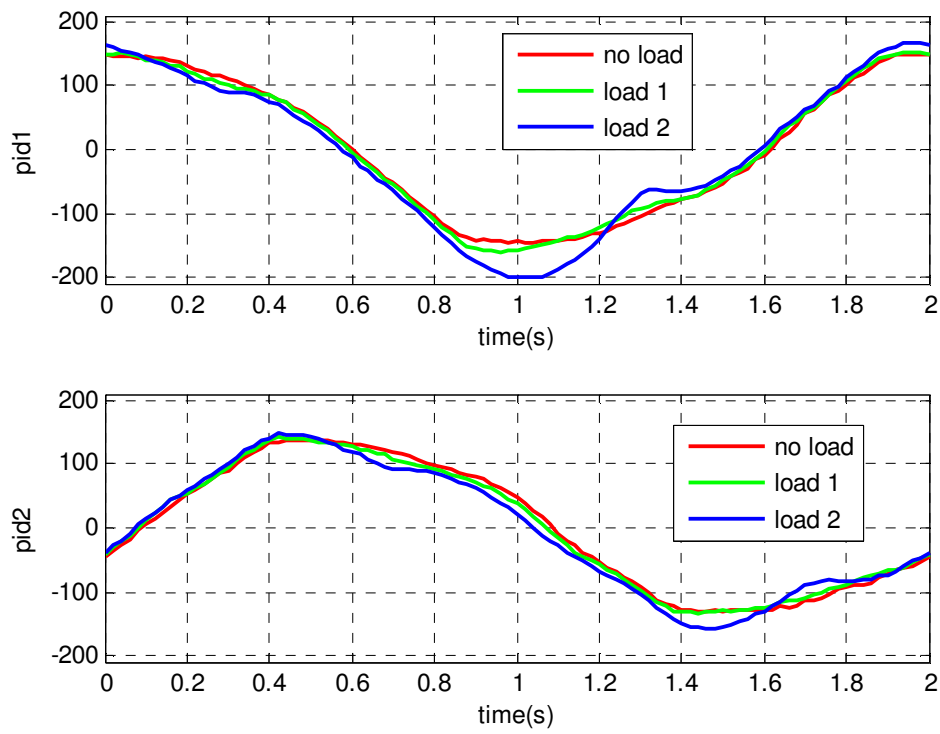

Figure 6.33: Controller output for different load on a wider trajectory. 
The effect of the HA1 reaching the saturation is seen in Fig. 6.34, where, in that instant, the deviation increases.
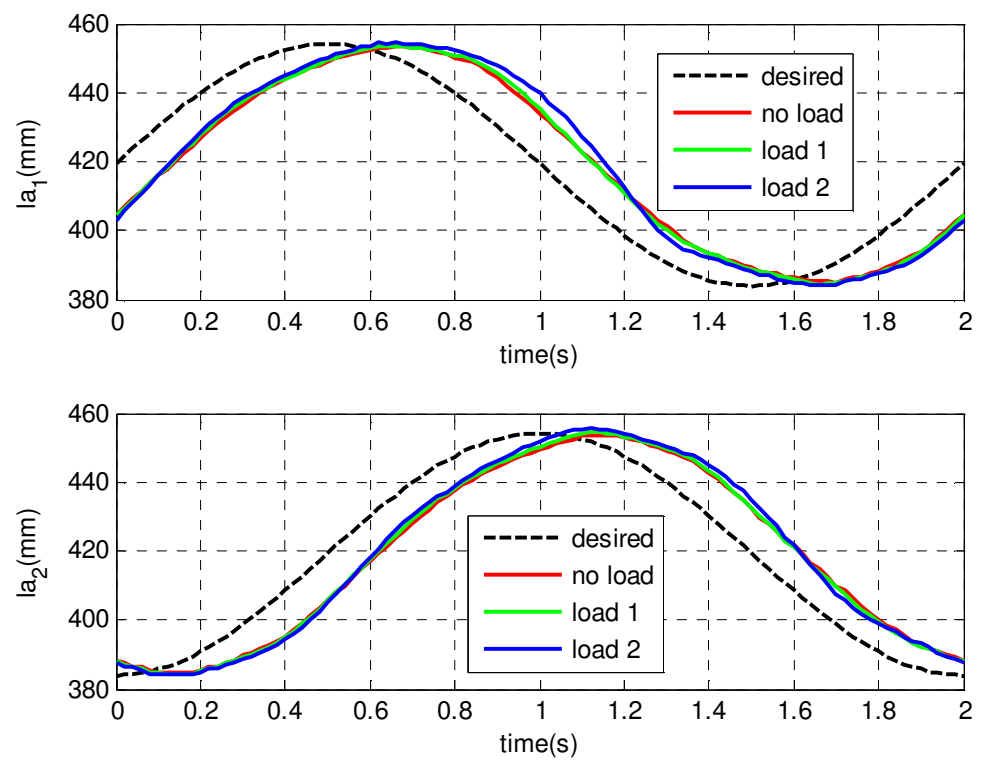

Figure 6.34: Actuators displacement for different loads on a wider trajectory.

Increasing even more the load, the system performance would degrade quickly, because of the controller saturation. Further tests with wider angles are not done because it compromises the stability. If the platform reaches the boundary with high speed and that large inertia, the impact may damage the system.

\section{5 .}

\section{Controller}

In the previous sections it was shown that the modeling gives a good insight to the behavior of the system. Indeed, the simulation predicts well the performance of the proposed system. Therefore, with the validated numerical simulation, the controller improvements are tested in the computer before the implementation in the platform. This procedure assures that the novel control will be stable. It also saves time, because the numerical test is faster than an experimental one. It is important to assure stability, because instabilities may 
damage the platform. After trying several controllers, the control parameters of the best result were validated experimentally.

The controller in all simulations and experiments presented in the previous section is a full proportional control with gain $k_{p}=1.00 \cdot 10^{5}$. After several attempts, an enhanced performance is achieved with the proportional gain $k_{p}=1.50 \cdot 10^{5}$ and an integral gain $k_{i}=2.00 \cdot 10^{3}$. The overshoots are reduced with an anti-windup control to reset the integral when the controller output is over a certain limit. Figure 5.35 shows a top view of the table center trajectory of this PI controller.

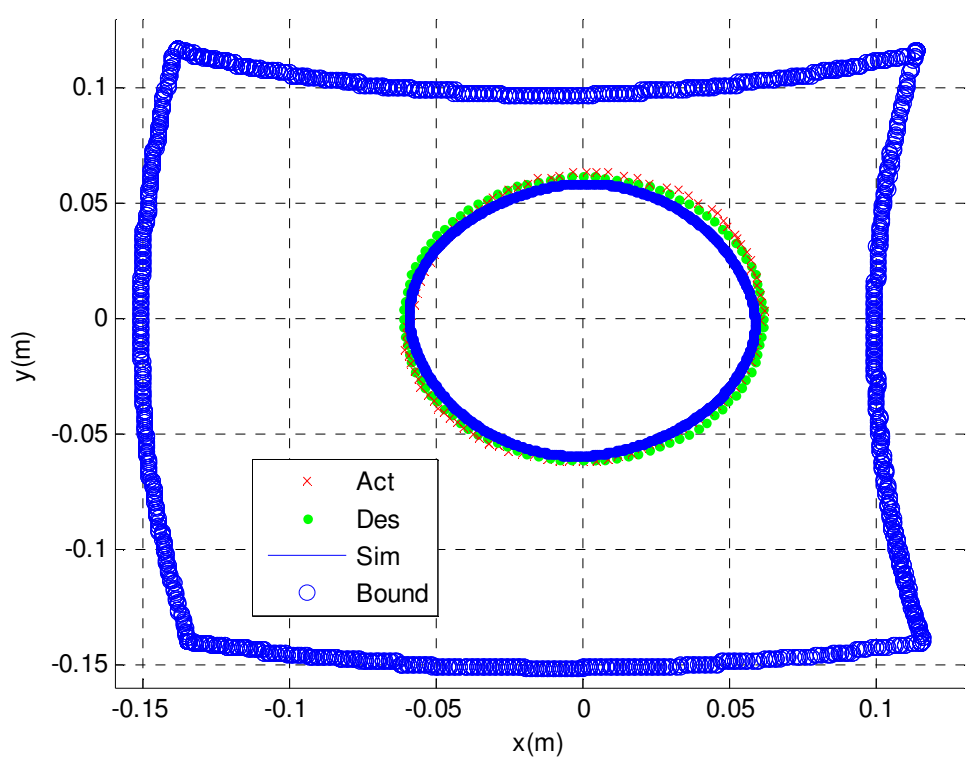

Figure 6.35: Table center trajectory with the PI controller.

Figure 6.36 presents the angular position error comparison between the $\mathrm{P}$ controller and the novel PI controller. 

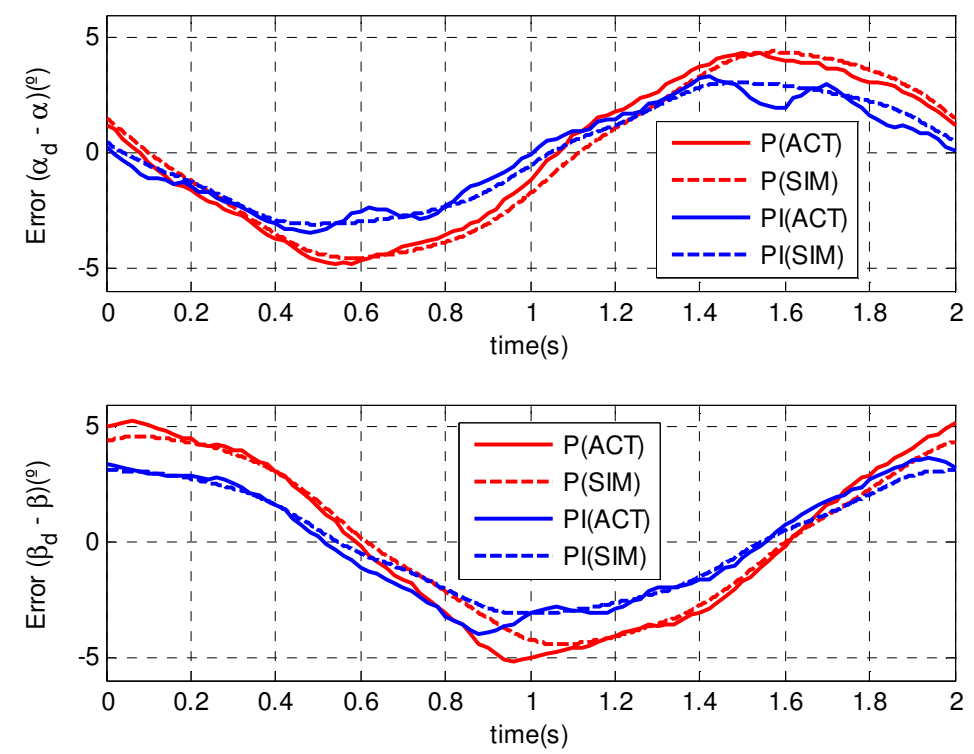

Figure 6.36: Angular position error.

Since it is a decentralized controller, each actuators length error is computed independently in the controller algorithm. Figure 6.37 presents the actuators error comparison between the PI controller and the P controller.
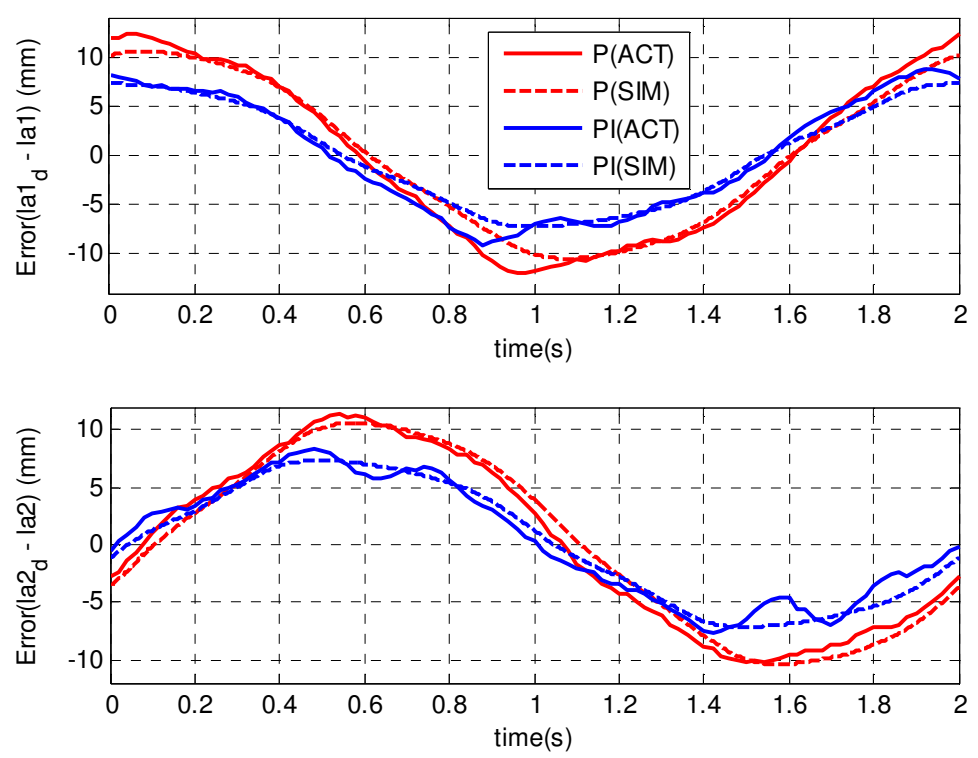

Figure 6.37: Actuators length error. 
These figures show the enhanced performance of the PI controller. The maximum error of the novel controller is about $30 \%$ smaller. And this PI controller is still very simple.

More sophisticated controller algorithms can be tried; however, the idea of this section is to present an approach to evaluate a new controller with reliable numerical results. Controllers with unstable results were not implemented in this method, avoiding the collision of the platform with its boundaries. 


\section{7 Conclusion and suggestions for future work}

In this work, two configurations of a 2-dof electrohydraulic actuated platform were developed for a low-cost TVC system. The most widespread TVC solution for liquid-propellant rockets is investigated using a gimbaled platform. In addition, the use of a $\mathrm{CVJ}$ as support for the moving table was validated as an alternative for the gimbal.

In a CVJ, the force is distributed in parallel between the spheres. In addition, there is no kinematic restriction for the amount of spheres. Therefore, the increase of the amount of spheres lead to a redundancy in the force distribution and a bigger thrust force capability. In a gimbaled device, the force distribution is serial, e.g. all forces are transmitted from one fork to the crosshead and from this to the other fork. Hence, a mechanism using a CVJ is able to deal with a bigger thrust force, thus it could replace the elastic joint used in solid-propellant rockets. The benefit of a CVJ over an elastic joint is the bigger joint angle capability. Indeed, the advantage of a gimbaled mechanism is the possibility to use encoders to measure the angular position. In a TVC with a CVJ or with an elastic joint, the actuators position could be measured for control purpose, or as shown in this work an IMU could be used.

The kinematic and the dynamic modeling of both platform versions were presented. In the homokinetic platform kinematics an approach to study CVJ is introduced. The models of the electrohydraulic system and the controller were also shown. These models were used to perform numerical simulations. The comparison between the numerical and the experimental results reveal that the presented model provides a good insight into the behavior of the system.

Traditionally, the position control of a hydraulic control system requires a servovalve; however, in this project the commercial valve was out of budget. Therefore, this Thesis proposed a novel hydraulic control valve built specially for the control of the platform. It provided a feasible electrohydraulic control system. The operation of the novel control valve was validated to control the actuators 
position of the platform. Finally, it is concluded that, in spite of the low bandwidth, the proposed hydraulic control system is able to control a low-cost TVC system. This approach is interesting for upper stage TVCs in vacuum, where high precision, and not velocity is the challenge.

The homokinetic platform proposed the replacement of the gimbal that supports the moving table by a CVJ. In the gimbaled platform, the attitude of the moving table is measured with rotary encoders. The homokinetic platform demands a different solution: an attitude estimation system. The system uses an IMU and a CF based on the orientation matrix. First, the real-time attitude estimation system is implemented in the gimbaled platform to validate the estimation using the rotary encoders data. The validation shows $1.3^{\circ}$ of maximum angular error, and $0.2^{\circ}$ in the average, of the estimated attitude in the encoder axis. Hence, the attitude estimation system was implemented for the homokinetic platform to measure its angular position.

The experimental validation showed that both platforms with the proposed control valve achieved good position tracking without instabilities during the tests. The performance of both platforms is similar. In spite of the similarity, the position error of the homokinetic platform carries the error of the attitude estimation, leading to a worse performance. However, the angular position measurement system of the gimbaled platform is thirty times more expensive than the attitude estimation system of the homokinetic configuration.

The behavior analysis of the gimbaled platform over different load conditions reveals the robustness of the hydraulic control system. The position tracking performance remains reliable, even when the load is substantially increased. The simple $\mathrm{P}$ controller demands the same opening for the valves, almost independent from the load on the actuators. This desirable characteristic is due to the proportional control valve manufactured for this Thesis. The commercial proportional control valves have two unwanted features: hysteresis and dead-band. But the proposed valve is driven by a stepper motor, instead of the solenoids of the commercial valve. The open-loop stepper motor digital control leads the valve to a tiny dead band and a small hysteresis.

After validating the numerical simulations and testing the hydraulic control system, an improved controller is proposed. The idea is to illustrate an application of the numeric simulation to evaluate novel control strategies. This demonstration 
begins proposing several setups of PI controllers with anti-windup and testing them with numerical simulation. After that, the controller with minimum position tracking error was implemented experimentally. It reveals that the proposed PI controller reduces the maximum error in $30 \%$. The method is a safe strategy, avoiding experimental tests with unstable controllers. The numerical implementation of a controller is faster than experimental trials, thus this method also saves time.

\section{1.}

\section{Future works}

Much of the time spent on this Thesis was dedicated to the construction of the experimental test bench. The novel control valve, DAQ and peripheral devices required much time to be functional. A significant part of time also was spent on the preliminaries test benches to identify the actuators friction and valve model. Now with everything finished a lot can be done to improve the platform performance and the accuracy of the modeling.

In this Thesis the pipeline losses are not considered in the numerical simulations, because of convergence difficulties in numerical simulations. As a future work, the linearization of some parts of the model, e.g. the pressure drop in the valve, should by tried to work around this issue.

In this work a decentralized PI controller achieves a stable position tracking, however a more sophisticated algorithm should improve the performance. Based on the result for parallel manipulators showed by Taghirad [34], the robust inverse dynamic control achieves an improved performance. In the literature gravity compensation [40] and self-tuning PD computed torque [41] have shown an enhanced performance. Therefore, the future works should try these control techniques to improve the accuracy.

A significant part of nonlinear behavior of the proposed platform is due to the actuators friction. In this Thesis, the actuator friction is considered speed dependent only. In future, a more precise model, e.g. the LuGre friction model [38], could be used for real-time compensation.

In future, the performance of the PDHV could be improved through a closed loop control of the stepper motor. As an alternative for an angular transducer 
feedback, a sensorless stepper control could improve the performance without a significantly increased cost [42-45]. 


\section{Bibliography}

[1] Li, Y.; Lu, H.; Tian, S.; Jiao, Z.; Chen, J.T.. Posture Control of Electromechanical-Actuator-Based Thrust Vector System for Aircraft Engine. IEEE Transactions on Industrial Electronics. Vol. 59, No. 9, 3561-3571, 2012.

[2] Wekerle, T.; Barbosa, E.G.; Batagini, C.M.; Costa, L.E.V.L.; Trabasso, L.G.. Brazilian Thrust Vector Control System Development: Status and Trends. Propulsion and Energy Forum, 52nd AIAA/SAE/ASEE Joint Propulsion Conference, Salt Lake City, UT, 2016.

[3] Li, Y.; Mathematical modeling and characteristics of the pilot valve applied to a jet-pipe/deflector-jet servovalve. Sensors and Actuators A 245 150-159, 2016.

[4] Wekerle, T.; Brito, A.; Trabasso, L.G.. Requirements for an Aerospace Actuation System derived from the Control Design Point of View, 23rd ABCM International Congress of Mechanical Engineering, Rio de Janeiro, RJ, Brazil, December 6-11, 2015.

[5] Huzel, D.K.; Huang, D.H.; Design Of Liquid Propellant Rocket Engines. Rocketdyne Division, North American Aviation, Inc., NASA, 1967.

[6] Brito, A.G.. Characterization of Electrohydraulic Actuators: Technical and Experimental Aspect. Experimental Techniques, Society for Experimental Mechanics, 2015.

[7] Lazic, D.V.; Ristanovic, M.R.. Electrohydraulic thrust vector control of twin rocket engines with position feedback via angular transducers. Control Engineering Practice 15, 583-594, 2007.

[8] Wekerle, T.; Barbosa, E.G.; Batagini, C.M.; Costa, L.E.V.L.; Trabasso, L.G.. Closed-loop actuator identification for Brazilian Thrust Vector Control development. Proceedings of the 20th IFAC Symposium on Automatic Control in Aerospace, Sherbrooke, Quebec, Canada, 2016.

[9] Ghosh, B.B.; Sarkar, B.K.; Saha, R.. Realtime performance analysis of different combinations of fuzzy-PID and bias controllers for a two degree of freedom electrohydraulic parallel manipulator. Robotics and ComputerIntegrated Manufacturing 34 62- 69, 2015.

[10] Manring, N.. Hydraulic control systems. John Wiley \& Sons, New Jersey, USA, 2005.

[11] Yu, J.; Zhuang, J.; Yu, D.. Modeling and Analysis of A Rotary Direct Drive Servovalve. Chinese Journal Of Mechanical Engineering, Vol. 27, No. 5, 2014.

[12] Cantelli, L.; Muscato, G.; Nunnari, M.; Spina, D.. A Joint-Angle Estimation Method for Industrial Manipulators Using Inertial Sensors. IEEE/ASME Transactions On Mechatronics, Vol. 20, No. 5, 2015.

[13] Ramaswamy, B.. Kalman Filter Based Estimation Of Inertial Measurement Unit Parameters In A Portable Biomechanical Assessment 
Suite (PBAS). The Pennsylvania State University The Graduate School College of Engineering, Thesis in Electrical Engineering, 2011.

[14] Kraft, E.. A Quaternion-based Unscented Kalman Filter for Orientation Tracking. Physikalisches Institut, University of Bonn, (2003).

[15] Edwan, E.; Knedlik, S.; Loffeld, O.. Constrained Angular Motion Estimation in a Gyro-Free IMU. IEEE Transactions On Aerospace And Electronic Systems Vol. 47, No. 1, 2011.

[16] McGinnis, R.S.; Perkins, N.C.. Inertial sensor based method for identifying spherical joint center of rotation. Journal of Biomechanics 46, 2546 $-2549,2013$.

[17] Rosario, M.B.D.; Lovell, N.H.; Redmond, S.J.. Quaternion-Based Complementary Filter for Attitude Determination of a Smartphone. IEEE Sensors Journal, Vol. 16, No. 15, 6008-6017, 2016.

[18] Tian, Y.; Wei, H.; Tan, J.. An Adaptive-Gain Complementary Filter for Real-Time Human Motion Tracking With MARG Sensors in Free-Living Environments. IEEE Transactions On Neural Systems And Rehabilitation Engineering, Vol. 21, No. 2, 254-264, 2013.

[19] Ghanbari, M.; Yazdanpanah, M.J.. Delay Compensation of Tilt Sensors Based on MEMS Accelerometer Using Data Fusion Technique. IEEE Sensors Journal, Vol. 15, No. 3, 1959-1966, 2015.

[20] Cockcroft, J.; Muller, J.H.; Scheffer, C.. A Complementary Filter for Tracking Bicycle Crank Angles Using Inertial Sensors. Kinematic Constraints, and Vertical Acceleration Updates, IEEE Sensors Journal, Vol. 15, No. 8, (8), 4218-4225, 2015.

[21] Metni, N.; Pflimlin, J.M.; Hamel, T.; Souères, P.. Attitude and gyro bias estimation for a VTOL UAV. Control Engineering Practice 14, 1511-1520, 2006.

[22] Fourati, H.. Heterogeneous DataFusion Algorithm for Pedestrian Navigation via Foot-Mounted Inertial Measurement Unit and Complementary Filter. IEEE Transactions On Instrumentation And Measurement, Vol. 64, No. 1, 221-229, 2015.

[23] Andersson, S.; Yan, L.. Capture of a Walker's Leg Using Inexpensive Inertial Sensors with Optimized Complementary Filter Design. (IJACSA) International Journal of Advanced Computer Science and Applications, Vol. 6, No. 1, 177-181, 2015.

[24] Pennestrì, E.; Rossi, V.; Salvini, P.; Valentini, P.P.; Pulvirenti, F.. Review and kinematics of Rzeppa-type homokinetic joints with straight crossed tracks. Mechanism and Machine Theory 90, 142-161, 2015.

[25] Kimata, K.; Nagatani, H.; Imoto, M.. Analysis of Ball-Type ConstantVelocity Joints Based on Dynamics. JSME International Journal, Vol. 47, No. 2, 736-745, 2004.

[26] Kimata, K.; Nagatani, H.; Imoto, M.; Kohara, T.. Numerical Analyses and Experiments on the Characteristics of Ball-Type Constant-Velocity Joints. JSME International Journal, Vol. 47, No. 2, 746-754, 2004. 
[27] Watson, I.; B. Prusty, G.; Olsen, J.. Conceptual design optimisation of a constant-velocity coupling. Mechanism and Machine Theory 68 18-34, 2013.

[28] Seherr-Thoss, H.C.; Schmelz, F.; Aucktor, E.. Universal Joints and Driveshafts. 2.ed; Berlin; 2006; 351p.

[29] Cardozo, W; Weber, H.. Analysis and develoment of a parallel 2-dof manipulator. 23rd ABCM International Congress of Mechanical Engineering; Rio de Janeiro, 2015.

[30] Cardozo, W; Weber, H.. Numerical and Experimental Analysis of a Parallel 2 DOF Manipulator. DINAME 2017 - Proceedings of the XVII International Symposium on Dynamic Problems of Mechanics; ABCM; São Sebastião, SP, Brazil; 2017

[31] Cardozo, W; Weber, H.. Dynamic modeling of a 2-dof parallel electrohydraulic-actuated homokinetic platform. Mechanism and Machine Theory 118, 2017.

[32] Wekerle, T.. Technology nationalization framework: nationalization and industrialization of high technology products for the Brazilian space sector. Thesis of Doctor of Science - Aeronautics Institute of Technology, São José dos Campos, 2016, 164p.

[33] Wang, Z.; Jia, Y.; Jin, L.; Duan, J.. Thrust vector control of upper stage with a gimbaled thruster during orbit transfer. Acta Astronautica 127, 2016 359-366.

[34] Taghirad, H.. Parallel robots: mechanics and control. Ed. CRC Press, Ney York, NY, 2013, 533p.

[35] Weber, H.I.. Raciocinando Dinâmica de Rotação. Book under development, not published yet, 2017.

[36] Jelali, M.; Kroll, A.. Hydraulic Servo-systems Modelling, Identification and Control. Springer, London, 2nd edition, 2003.

[37] Piatkowski, T.. Dahl and LuGre dynamic friction models - The analysis of selected properties. Mechanism and Machine Theory 73, 2014, 91100.

[38] Yao, J.; Deng, W.; Jiao, Z.. Adaptive Control of Hydraulic Actuators With LuGre Model-Based Friction Compensation. IEEE Transactions On Industrial Electronics, Vol. 62, No. 10, 2015.

[39] Moody, L.F.. Friction Factors for Pipe Flows. Transactions of the A.S.M.E. 66, 1944, 671-684.

[40] Yang, C.; Huang, Q.; Jiang, H.; Peter, O.; Han, J.. PD control with gravity compensation for hydraulic 6-DOF parallel manipulator. Mechanism and Machine Theory 45, 2010, p.666-677.

[41] Le, T.; Kang, H.; Suh, Y.; Ro, Y.. An online self-gain tuning method using neural networks for nonlinear PD computed torque controller of a 2dof parallel manipulator. Neurocomputing 116, 2013, p.53-61.

[42] Liu, J.; Cheng, K. W. E.; To, S.; Dual Closed Loop Controller of Bus Stepper Motor Based on Back-EMF. 3rd International Conference on Power Electronics Systems and Applications; 2009. 
[43] Bendjedia, M.; Ait-Amirat, Y.; Walther, B.; Berthon, A.; Sensorless Controlo of Hybrid Stepper Motor. European Conference on Power Electronics and Applications; 2-5 September, 2007.

[44] Bendjedia, M.; Ait-Amirat, Y.; Walther, B.; Berthon, A.; DSP Implementation of Rotor Position Detection Method for Hybrid Stepper Motors; Power Electronics and Motion Control Conference. p. 1-5; 14-16 August, 2006.

[45] Obermeier, C.; Kellermann, H.; Brandenburg, G.; Sensorless Field Oriented Speed Control of a Hybrid and a Permanent Magnet Disk Stepper Motor Using an Extended Kalman Filter. Electric Machines and Drives Conference Record; Milwaukee, WI; May, 1997. 


\section{A \\ Preliminary test benches}

\section{A.1 No load test bench}

As a first trial for the electrohydraulic control system, an actuator with no load is considered. Figure A.1 presents a photo of this system.

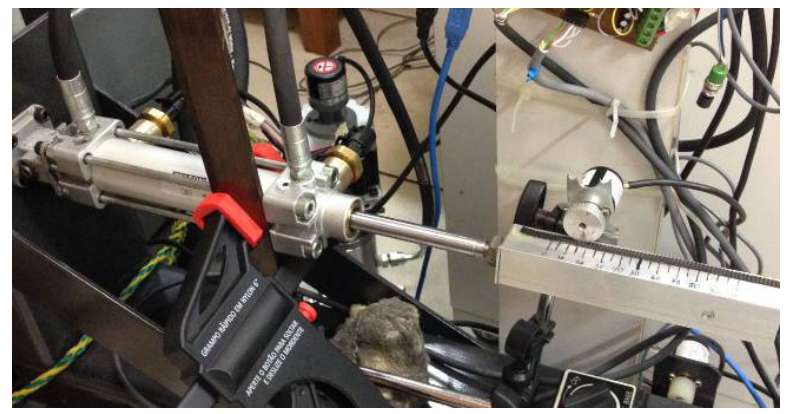

Figure A.1: No load test bench.

This experiment shows that the PDHV is able to control the actuator without a load. In this test bench, a rotary encoder with a rack pinion arrangement measures the linear displacements of the actuator. Pressure transducers measure the actuator chambers pressure and the supply pressure. And an encoder measures the angular position of the spool of the PDHV. The DAQ system sends the data to a Matlab script that runs a control algorithm. After processing these input signals, the output control variable is sent back to the DAQ. This DAQ has a valve controller.

\section{A.2}

Inverted pendulum test bench

After checking that the proposed control system is able to control a no load system, a load is proposed. As the 2-DOF platform load is composed largely by a 
gravitational component, a test bench with a large gravity component is proposed. The inverted pendulum built for this purpose is shown in Fig. A.2.

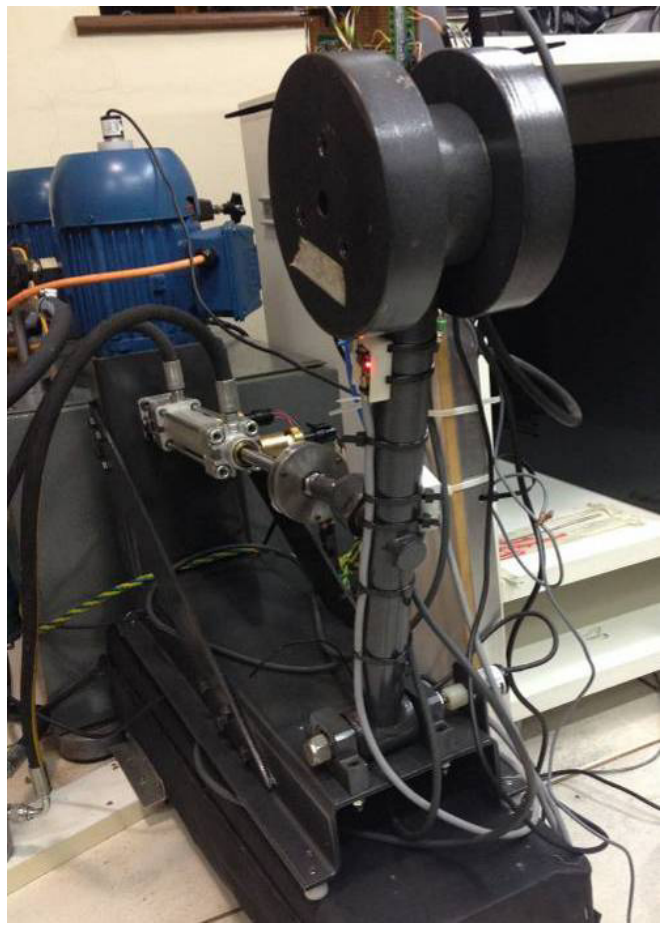

Figure A.2: Inverted pendulum test bench.

A rotary encoder measures the angular position of the inverted pendulum. A load cell measures the force done by the actuator. Two IMUs measure the linear acceleration and the angular velocity of two points. This test bench shows a good position tracking of the system with load.

\section{A.3 Actuator friction identification}

Besides testing the system with and without a load, these two preliminary test benches are used to quantify the friction force on the actuator. This is important to perform simulations and it is not provided by the manufacturer. A direct friction measurement is difficult, hence all other components of the actuator forces are measured. The difference between the sum of these components and the total force is the estimated friction. This is the most widespread method to measure the hydraulic actuator friction; however, the condition of the experiment 
varies a lot. Figure A.3 shows the friction estimation with four different conditions for the same actuator:

- No load test bench, and a desired sinusoidal trajectory with $1 \mathrm{~Hz}$ frequency and $30 \mathrm{~mm}$ of amplitude (red dots);

- Inverted pendulum test bench, and a desired sinusoidal trajectory with $1 \mathrm{~Hz}$ frequency and $10^{\circ}$ of amplitude (blue dots);

- Inverted pendulum test bench, and a square wave as desired trajectory with $0.1 \mathrm{~Hz}$ frequency and $10^{\circ}$ of amplitude (black dots);

- No load test bench under constant speed (green dots).

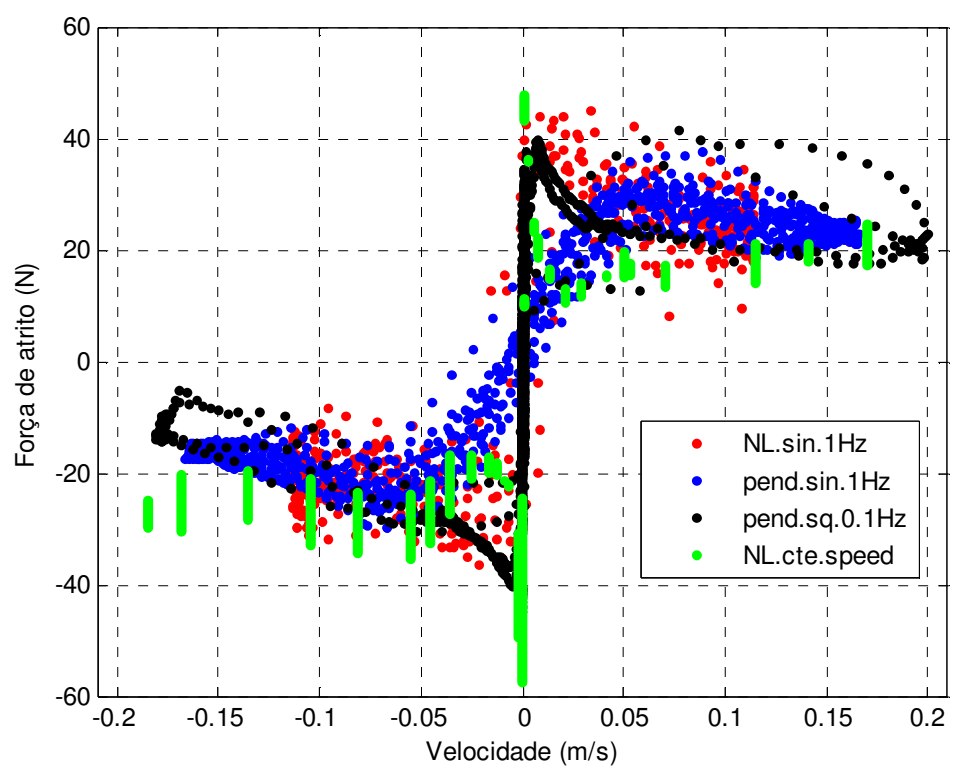

Figure A.3: Four different friction estimations.

Hence, these four different measures give four different results for the same actuator. As presented by [37] and [38], the friction is time dependent. Therefore, the difference between the experiments agrees with them showing that friction is not speed dependent only. However, in this Thesis, it is considered that friction is a speed function only, due to the simplicity of the model. 
B

\section{Electrohydraulic circuit}

In this section, the electrohydraulic system is described. Figure B.1 present a diagram.

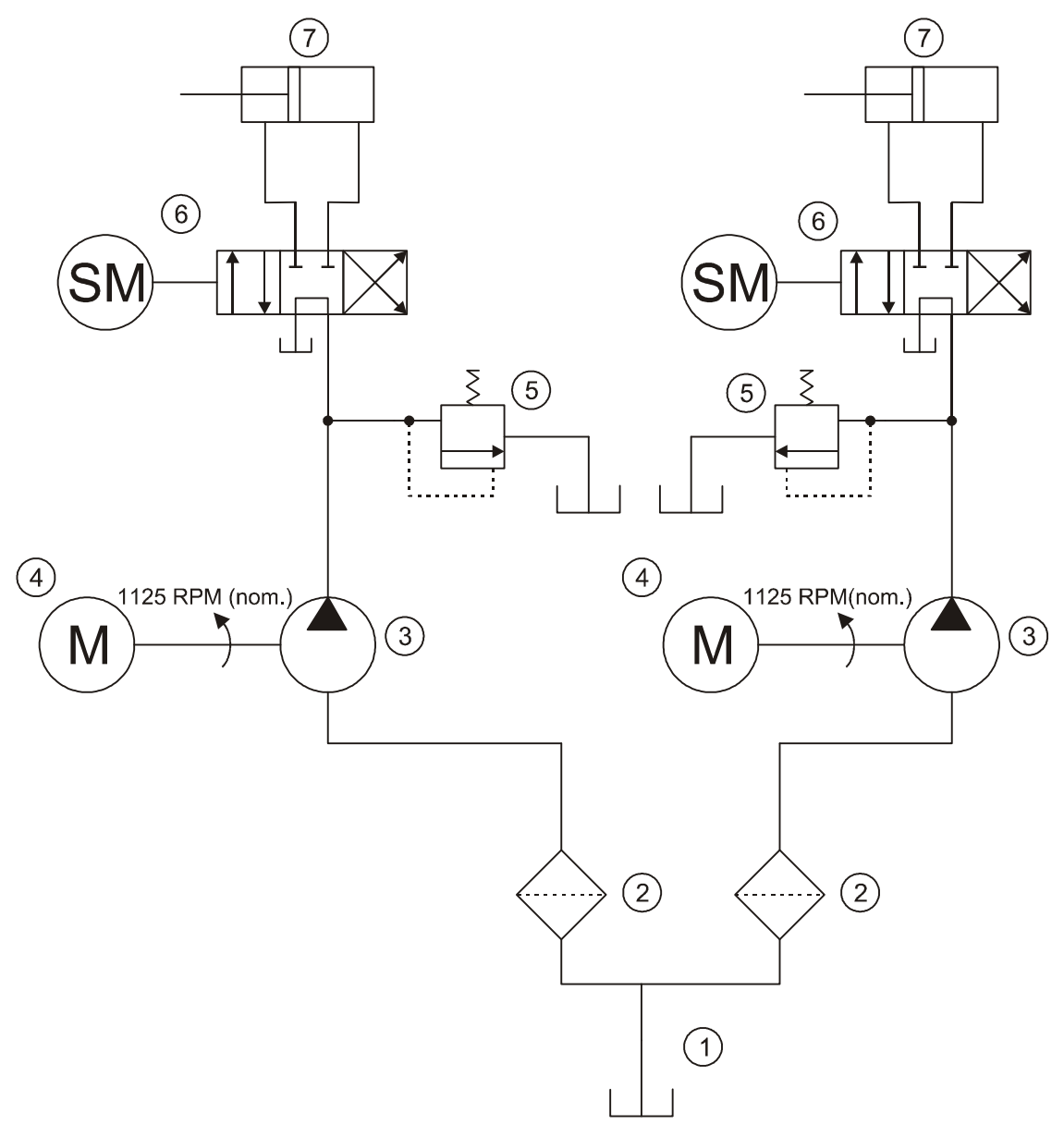

Figure B.1: Electrohydraulic diagram.

Where the numbers on this figure represents:

1. Steel tank (or reservoir) with 70 liters of Castrol Hyspin AWS 32.

2. Suction Filter HDA FTS - 45

3. Vane Pump EATON V10-1P3P-1A-20

4. AC Motor Weg 1,1 kW 6P 90S 220V B5 
5. Relief Valve CT-06-B-50 with the pressure range between 125 psi and $1.000 \mathrm{psi}$.

6. Proportional Digital Hydraulic Valve (PDHV). It is a rotary spool 4 ways valve with the stepper motor Akiyama AK85H/3.5-1.8 driving the rotary spool.

7. Linear hydraulic actuator. Built by Belton with the code APBS032D-Z01. It has $100 \mathrm{~mm}$ stroke, $32 \mathrm{~mm}$ embolus diameter and $12 \mathrm{~mm}$ rod diameter. This actuator is a pneumatic one, with the sealing changed to hydraulic application. Hence, the pressure is limited to 30 bar.

The hydraulic unit also has a vertical manometer D.63 MV-63 70 bar glic. The coupling between pump and the AC motor is done with a HDA AC-28. The reservoir has a level display HDA VI76 and the air filter FA44-40/2. 


\section{C \\ Data acquisition system}

Figure C.1 depicts the input part of the DAQ system.

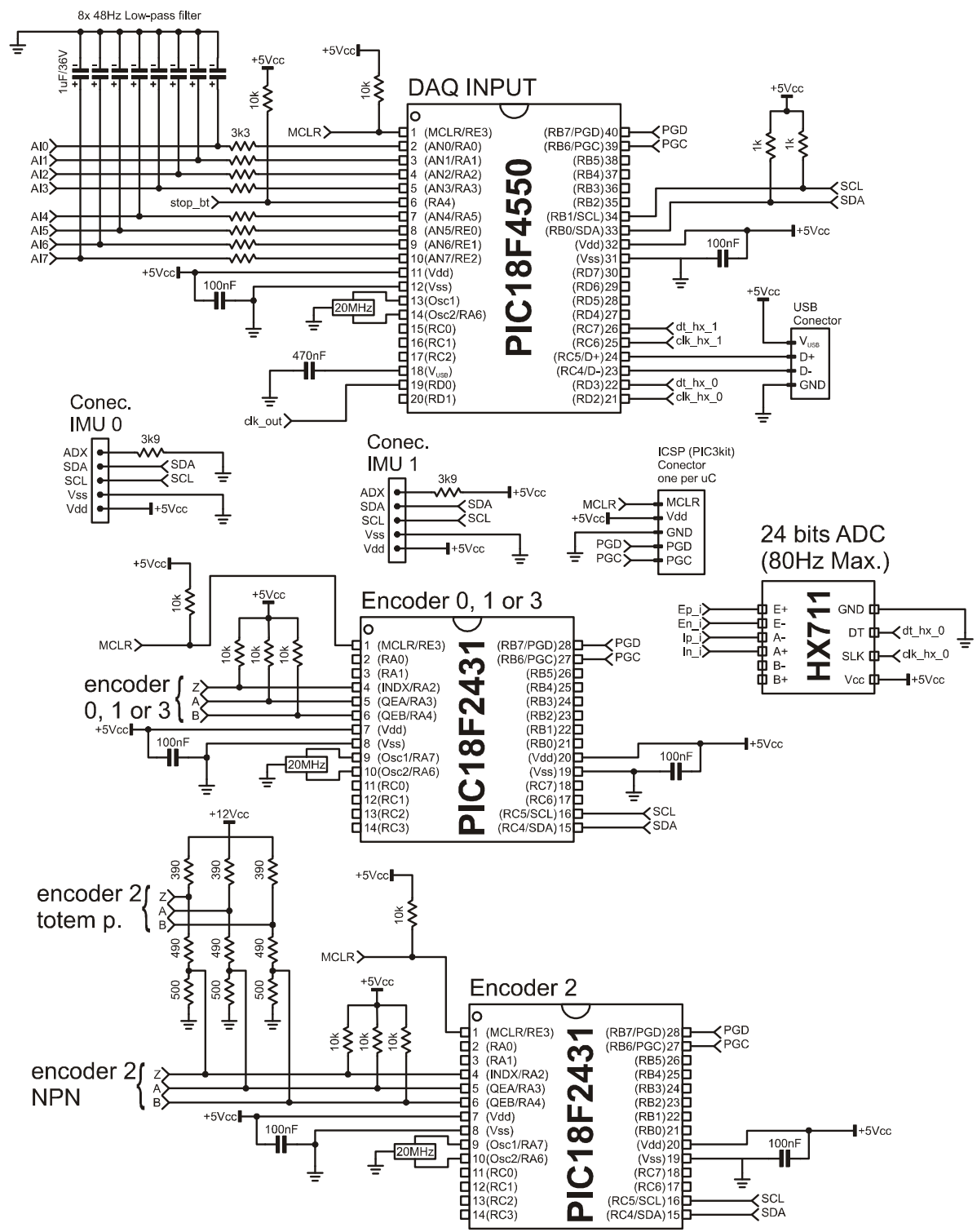

Figure C.1: DAQ input circuit. 
The PIC18F4550 microcontroller receives the data from two IMUs and from three incremental encoder microprocessors through an $\mathrm{I} 2 \mathrm{C}$ communication line. Eight 10 bits analog-digital converter (ADC) receive the low-pass filtered signals from pressure transducers. Two 24 bits ADC modules send the digitalized load cells signals to the microcontroller. All these data are sent though a Universal Serial Bus (USB) connection to a running script on a computer with Matlab®. This script stores the sensors data on vectors and runs the control algorithm. The output variables are sent to a microcontroller in the DAQ output circuit, depicted in Figure C.2.
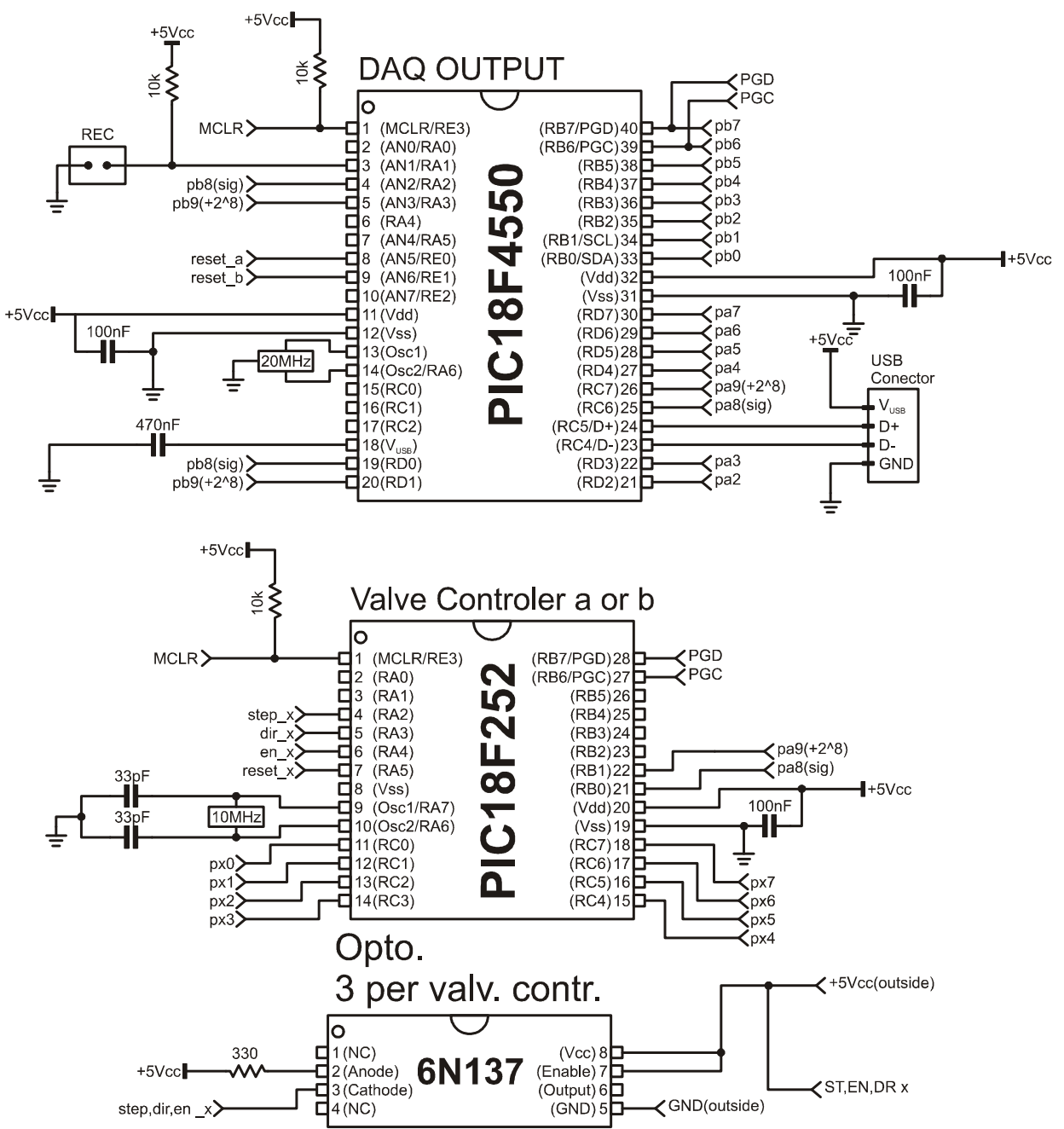

Figure C.2: DAQ output circuit.

The output variables received by the PIC18F4550 microcontroller are the demanded angular position for the PDHVs. These demanded positons are sent to 
valve controllers through a parallel connection. These valve controllers send the step and direction pulses to the drivers of the stepper motors on the PDHVs using an opto-isolator circuit. 


\section{D Rotation around a vector}

This appendix develops the rotation around a vector presented in Chapter 2. Indeed, this section gives an explanation for the Eq. (2.17). The basic idea is to rotate the vector $\boldsymbol{r}$ around the invariant vector $\boldsymbol{p}$ to obtain the vector $\boldsymbol{r}+$. The vector $r+$ is calculated through a geometrical inspection of the Fig. D.1.

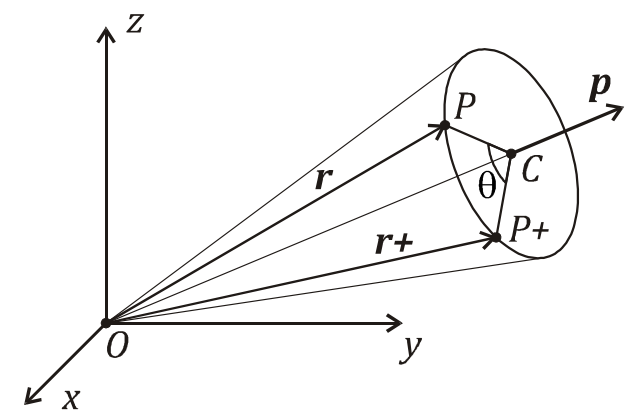

Figure D.1: Rotation around the Euler axis $\boldsymbol{p}$.

From this figure, it is concluded that:

$\boldsymbol{r}+={ }_{O} \boldsymbol{r}_{P+}={ }_{O} \boldsymbol{r}_{P}+{ }_{P} \boldsymbol{r}_{C}+{ }_{C} \boldsymbol{r}_{P+}$.

The vector ${ }_{o} \boldsymbol{r}_{P}$ is the vector $\boldsymbol{r}$, which is given, thus,

$$
{ }_{O} \boldsymbol{r}_{P}=\boldsymbol{r} \text {. }
$$

Figure D.2 depicts the triangle formed by the point $P P+C$, where $R$ is the radius of circle presented in Fig. D.1. 


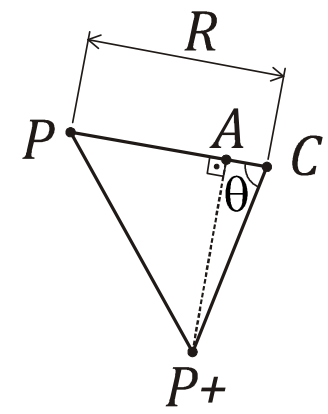

Figure D.2: $P P+C$ triangle.

The $P P+C$ triangle presents and alternative way for the path proposed by Eq. (D.1), using the point $A$, the $\boldsymbol{r}+$ is given by,

$$
\boldsymbol{r}+{ }_{O} \boldsymbol{r}_{P}+{ }_{P} \boldsymbol{r}_{A}+{ }_{A} \boldsymbol{r}_{P+} .
$$

The magnitude of the vector ${ }_{A} \boldsymbol{r}_{P+}$ is given by right triangle formed by the point $A P+C$,

$\left|{ }_{A} \boldsymbol{r}_{P+}\right|=R \sin \theta$.

The direction of the vector ${ }_{A} \boldsymbol{r}_{P+}$ is parallel to the vector product $\tilde{\boldsymbol{p}} \boldsymbol{r}$. Since $\boldsymbol{p}$ is unitary, the magnitude is,

$$
|\tilde{p} \boldsymbol{r}|=|\boldsymbol{r}| \sin \gamma .
$$

where $\gamma$ is the angle between $\boldsymbol{p}$ and $\boldsymbol{r}$. Looking at the $O C P$ triangle of Fig. D.3, an alternative expression for the $\tilde{\boldsymbol{p}} \boldsymbol{r}$ is obtained.

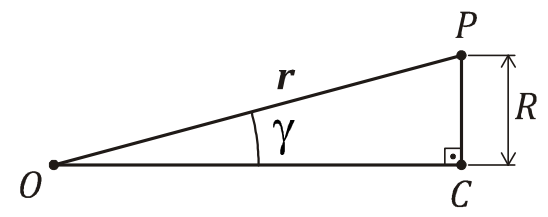

Figure D.3: $P P+C$ triangle.

From Fig. D.3,

$|\boldsymbol{r}| \sin \gamma=R$.

Thus,

$$
{ }_{A} \boldsymbol{r}_{P+}=\left|{ }_{A} \boldsymbol{r}_{P+}\right| \frac{\tilde{\boldsymbol{p}} \boldsymbol{r}}{R}=R \sin \theta \frac{\tilde{\boldsymbol{p}} \boldsymbol{r}}{R}=\sin \theta \tilde{\boldsymbol{p}} \boldsymbol{r} .
$$

Looking at Fig. D.2, the magnitude of the vector ${ }_{P} \boldsymbol{r}_{A}$ is given by,

$$
\left|{ }_{P} \boldsymbol{r}_{A}\right|=R-R \cos \theta=(1-\cos \theta) R \text {. }
$$


The vector ${ }_{P} \boldsymbol{r}_{A}$ is orthogonal to the vector ${ }_{A} \boldsymbol{r}_{P+}$ and to the vector $\boldsymbol{p}$.

${ }_{P} \boldsymbol{r}_{A}=\left.\right|_{P} \boldsymbol{r}_{A} \mid \tilde{\boldsymbol{p}}\left(\frac{\tilde{\boldsymbol{p}} \boldsymbol{r}}{R}\right)=(1-\cos \theta) R \tilde{\boldsymbol{p}}\left(\frac{\tilde{\boldsymbol{p}} \boldsymbol{r}}{R}\right)=(1-\cos \theta) \tilde{\boldsymbol{p}}^{2} \boldsymbol{r}$

Hence, all the terms of the Eq. (D.3) is known, thus,

$\boldsymbol{r}+=\boldsymbol{r}+(1-\cos \theta) \tilde{\boldsymbol{p}}^{2} \boldsymbol{r}+\sin \theta \tilde{\boldsymbol{p}} \boldsymbol{r}=\left(\boldsymbol{E}+(1-\cos \theta) \tilde{\boldsymbol{p}}^{2}+\sin \theta \tilde{\boldsymbol{p}}\right) \boldsymbol{r}$.

Therefore, the calculation of the Eq. (2.17) is explained. 